\title{
LEARNING METHODS FOR TEMPORAL-SPATIAL OPPORTUNISTIC SPECTRUM ACCESS IN COGNITIVE RADIO NETWORKS
}

\author{
ZHANG YI
}

School of Electrical and Electronic Engineering

A thesis submitted to the Nanyang Technological University in partial fulfilment of the requirement for the degree of

Doctor of Philosophy 


\title{
Learning Methods For Temporal-Spatial Opportunistic \\ Spectrum Access In Cognitive Radio Networks
}

\author{
by \\ Zhang Yi \\ submitted to the Nanyang Technological University \\ in partial fulfilment of the requirements for the degree of \\ Doctor of Philosophy \\ January, 2016
}

\begin{abstract}
As a natural resource, the radio spectrum is usually regulated by government agencies and static spectrum allocation policies are widely adopted by most countries. With the increasing popularity of mobile devices and need for high speed data transmission, static spectrum allocation policies can no longer satisfy all demands for spectrum.

In cognitive radio networks (CRNs), opportunistic spectrum access (OSA) alleviates the spectrum under-utilization problem by allowing unlicensed secondary users (SUs) to identify and exploit the unused spectrum owned by primary users (PUs) temporally and spatially while limiting the interference to PUs below a predefined threshold. Designing effective methods for temporal-spatial OSA is thus crucial for improving spectrum utilization nowadays.

We first consider the problem of estimating the no-talk region of the PU for temporal-spatial OSA, i.e., the region outside which SUs may utilize the PU's spectrum opportunistically regardless of whether the PU is transmitting or not. Based on a distributed learning framework, we propose a distributed boundary estimation
\end{abstract}


algorithm that allows SUs to determine the boundary of the no-talk region collaboratively through message passing between SUs. We analyze the trade-offs between estimation error, communication cost, setup complexity, throughput and robustness. Simulation results suggest that our proposed algorithm have lower estimation errors and better robustness compared to various other methods.

Within the no-talk region of the PU, SUs who do not interfere with each other can make use of the same PU channel. We then formulate and study a multi-user multi-armed bandit (MAB) problem that exploits the temporal-spatial OSA of PU channels for these SUs located inside the region. We first propose a centralized channel allocation policy for finding an optimal channel allocation and learning the statistics of the channels. We show that this policy is order-optimal with logarithmic regret, but requires solving a NP-complete optimization problem at exponentially increasing time intervals. To overcome the high computation complexity at the central processor, we also propose heuristic distributed learning policies that however have linear regrets. We compare the performance of our proposed policies with other distributed policies recently proposed for temporal (but not spatial) OSA. Simulation results suggest that our policies perform significantly better in terms of average regret than the benchmark policies.

Finally, we also propose three collaborative channel learning policies for temporalspatial OSA, which embed collaboration in the channel statistics learning process. We identify spectrum access opportunities via information exchange among neighboring SUs by applying consensus algorithms on their channel sensing observations, empirical estimates of channel idle probabilities and estimated channel ranks. We compare the performance of these policies with a distributed channel allocation policy. Simulation results suggest that our proposed collaborative policies outperform the distributed 
access rank learning policy which does not consider collaborations in the learning process.

Supervisor: Tay Wee Peng

Title: Assistant Professor, Nanyang Technology University Singapore

Supervisor: Dominique Gaïti

Title: Professor, University of Technology of Troyes France

Supervisor: Li Kwok Hung

Title: Associate Professor, Nanyang Technology University Singapore 


\section{Acknowledgements}

I would like to express my great gratitude to my main supervisors Professor Tay Wee Peng in Nanyang Technology University (NTU) for his continuous support, patience and encouragement. I am deeply touched by his passion in research, technical insights regarding my research area and invaluable guidance throughout my study journey. No matter how busy he was, he could try to arrange a weekly meeting for discussing the issues I encountered. Now matter how late it was, he could reply my question rapidly. He shared me his rich research experience and taught me how to write a good scientific paper. He actually treated me and my work seriously. I am very fortunate to be his student. I am also sincerely grateful to my NTU co-supervisor Professor Li Kowk Hung. He gave me many valuable suggestions, assisted me to find my research direction and helped me to build my self-confidence in research. His emphasis on clarity, preciseness and practicality in my work has helped me to achieve my academic goals. I would like to thank deeply to my supervisor Professor Dominique Gaïti when I was pursuing my study in University of Technology of Troyes (UTT) France. She helped me to smoothly engage with the life in France. And she tried to solve any of my queries timely and provided guidance whenever I met difficulties.

I would like to recognize NTU and UTT for offering me this precious opportunity to study under the joint Ph.D program. Thank Professor Moez Esseghir, Mrs. Chua-Goh Wei Jiuan, Ms. Koh-Ho Cheng Fiang Frances, Ms. Goh-Fong Lai Peng Karen, Madam Pascale Denis and Madam Isabelle Leclercq for their great help and encouragement. 
I am also thankful to all the lovely friends I met in NTU and UTT for their accompany and friendship, including Luo Wuqiong, Tang Jianhua, Liu Yongwen, Tang Wenyin, Shi Xiaomeng, Yang Xin, Amina, Cheng Chi, Leng Mei, Sun Meng, Francois and Wang Yuan. My postgraduate life become extremely colorful and enjoyable because of them.

Most importantly, I would like to thank my beloved family for their unconditional support. As a mother with a young kid, it is not easy for me to make a good balance between studying and taking care of the whole family. To finish my Ph.D study, my family and I made a lot of sacrifices and overcame many difficulties. I deeply thank my husband and parents-in-law for looking after my son when I studied overseas. I also thank my parents for their love and support. All their love is a source of energy and inspiration for me. 


\section{Contents}

1 Introduction $\quad 23$

1.1 Motivation and Objective $\ldots \ldots \ldots \ldots \ldots \ldots$

1.2 Contributions of the Thesis $\ldots \ldots \ldots \ldots \ldots$

1.2.1 Distributed Boundary Learning For Spectrum Sensing . . . . . 26

1.2.2 Channel Allocation and Learning For Temporal-Spatial Spectrum Reuse . . . . . . . . . . . . . . . . . . . . 28

1.2.3 Collaborative Learning For Temporal-Spatial Spectrum Reuse 29

1.3 Organization of the Thesis $\ldots \ldots \ldots \ldots \ldots \ldots$

2 Related Works 33

2.1 Spectrum Sensing and Boundary Estimation . . . . . . . . 35

2.2 Multi-armed Bandit. . . . . . . . . . . . . . . . 38

2.3 Collaborative Learning . . . . . . . . . . . . . . . . . 40

3 Distributed Boundary Learning for Spectrum Sensing 43

3.1 Problem Formulation . . . . . . . . . . . . . . . . . . 44

3.2 Distributed Boundary Estimation $\ldots \ldots \ldots \ldots \ldots \ldots$ 
3.2.1 Boundary Cluster Identification . . . . . . . . . . . . 52

3.2.2 Distributed Boundary Estimation . . . . . . . . . . . . . 53

3.3 Performance Analysis . . . . . . . . . . . . . . . . . . 60

3.3.1 Communication Cost and Estimation Error . . . . . . . . . . 61

3.3.2 Setup Complexity and Throughput . . . . . . . . . . . 69

3.4 Simulation Results . . . . . . . . . . . . . . . . . . . . 72

3.4.1 Estimation Error and Communication Cost. . . . . . . . 73

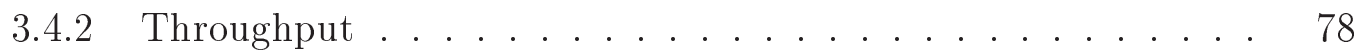

3.4.3 Robustness ...................... 79

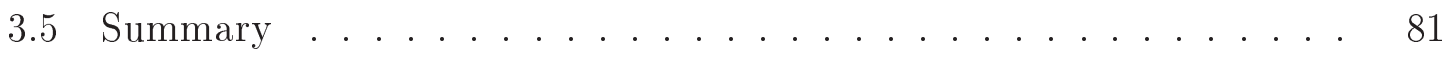

4 Channel Allocation and Learning For Temporal-Spatial Spectrum $\begin{array}{ll}\text { Reuse } & 85\end{array}$

4.1 Problem Formulation . . . . . . . . . . . . . . . . . . . 87

4.2 Centralized Channel Allocation Policy . . . . . . . . . . . . . . 92

4.3 Distributed Channel Access Ranking and Learning . . . . . . . . . . 98

4.3.1 CARL Policy . . . . . . . . . . . . . . . . . . 99

4.3 .2 DARL Policy . . . . . . . . . . . . . . . 102

4.3 .3 Regret Bounds . . . . . . . . . . . . . . . . 104

4.4 Simulation Results . . . . . . . . . . . . . . . . . . . . 108

4.4 .1 Small Size Graphs . . . . . . . . . . . . . . . . . . 108

4.4.2 Large Size Random Graphs . . . . . . . . . . . . . . . . . . . 111

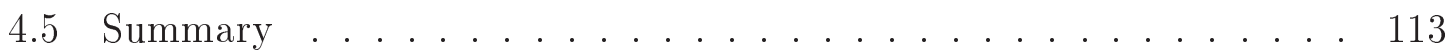


5.1 Problem Formulation . . . . . . . . . . . . . . . . . . . 118

5.2 Collaborative Channel Learning . . . . . . . . . . . . . . . . . . . 119

$5.2 .1 \quad$ CAOCL Policy . . . . . . . . . . . . . . . . . 119

$5.2 .2 \quad$ CAMCL Policy . . . . . . . . . . . . . . . . . 121

5.2 .3 CCRL Policy . . . . . . . . . . . . . . . . . . . 124

5.3 Simulation Results . . . . . . . . . . . . . . . . . . 127

5.3.1 Small Size Graphs _ . . . . . . . . . . . . . . . 127

5.3.2 Large Size Random Graphs _ . . . . . . . . . . . . . . 128

5.4 Summary . . . . . . . . . . . . . . . . . . . . . 131

6 Conclusion and Future Work 133

6.1 Conclusion . . . . . . . . . . . . . . . . . . . 133

6.2 Future Work . . . . . . . . . . . . . . . . . . . 135

$\begin{array}{ll}\text { A List of Publications } & 153\end{array}$ 
THIS PAGE INTENTIONALLY LEFT BLANK 


\section{List of Figures}

3-1 Spatial spectrum sharing between PU and multiple SUs. SU 1 and 2 can use the licensed spectrum of the PU without spectrum sensing. SU 3 can only utilize the spectrum when the $\mathrm{PU}$ is inactive. . . . . . . . 46

3-2 Normalized estimation errors and total communication costs for differ-

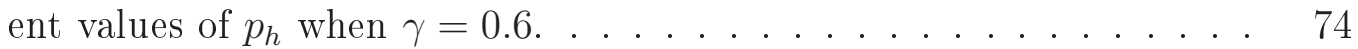

3-3 Normalized estimation errors and total communication costs when $p_{h}=$ 0.8 , and the threshold $\gamma$ is varied. . . . . . . . . . . 76

3-4 Weighted sum of communication cost and estimation error when $\gamma=$ 0.6 and $p_{h}=0.8$, with varying SU density. . . . . . . . . . .

3-5 Normalized estimation error versus number of inter-cluster communications. ......................... 77

3-6 ROC curves under FC and DBE-SS. . . . . . . . . . . . . . . . 78

3-7 PU throughput and throughput per SU under FC and DBE-SS. . . . 79

3-8 Average throughput per SU as volume of $A$ changes. . . . . . . . . . . 80

3-9 Robustness comparison of boundary estimation algorithms after flipping the observations. . . . . . . . . . . . . . . 
3-10 Robustness comparison of DBE algorithm with and without smoothing constraints after changing the density. . . . . . . . . . . . 82

3-11 Estimated boundaries with and without smoothing constraints. . . . . 82

4-1 Spatial spectrum reuse in a CRN with multiple SUs. . . . . . . . 86

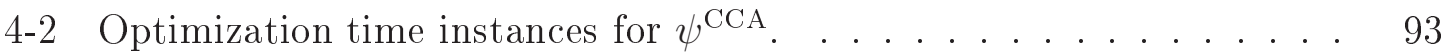

4-3 Interference graphs of three CRNs (a) a ring graph (b) a grid graph (c) a randomly generated graph. . . . . . . . . . . . . . . . . . 109

4-4 Normalized regret $\frac{R(n, \psi)}{\log n}$ vs. time slot $n$ on ring graph. . . . . . . . . 110

4-5 Normalized regret $\frac{R(n, \psi)}{\log n}$ vs. time slot $n$ on grid graph. . . . . . . . . 110

4-6 Normalized regret $\frac{R(n, \psi)}{\log n}$ vs. time slot $n$ on randomly generated graph. 111

4-7 Normalized regret $\frac{R(n, \psi)}{\log n}$ vs. time slot $n$ on ER graphs. . . . . . . . . 114

4-8 Normalized regret $\frac{R(n, \psi)}{\log n}$ vs. time slot $n$ on RC graphs. . . . . . . . . 114

5-1 An interference graph of a CRN with three SUs . . . . . . . . . 121

5-2 Normalized regret $\frac{R(n, \psi)}{\log n}$ vs. time slot $n$ with collaborative learning on ring graph. . . . . . . . . . . . . . . . . . . . . . . . . . . . . 128

5-3 Normalized regret $\frac{R(n, \psi)}{\log n}$ vs. time slot $n$ with collaborative learning on grid graph. . . . . . . . . . . . . . . . .

5-4 Normalized regret $\frac{R(n, \psi)}{\log n}$ vs. time slot $n$ with collaborative learning on randomly generated graph. . . . . . . . . . . . . . . . . . . . 129

5-5 Normalized regret $\frac{R(n, \psi)}{\log n}$ vs. time slot $n$ with collaborative learning on ER graphs. . . . . . . . . . . . . . . . . . . . . . 130 
5-6 Normalized regret $\frac{R(n, \psi)}{\log n}$ vs. time slot $n$ with collaborative learning on RC graphs. . . . . . . . . . . . . . . . . . 130 
THIS PAGE INTENTIONALLY LEFT BLANK 


\section{List of Tables}

3.1 Summary of notations used in Chapter $3 \ldots \ldots$. . . . . . 51

3.2 Summary of notations used for performance analysis in Chapter 3 . . 62

3.3 Simulation parameters in Chapter $3 \ldots \ldots$. . . . . . . . 73

4.1 Summary of notations used in Chapter $4 \ldots \ldots 2$

4.2 Performance of $\psi^{\mathrm{CARL}}$ and $\psi^{\mathrm{DARL}}$ in finding the correct chromatic num-

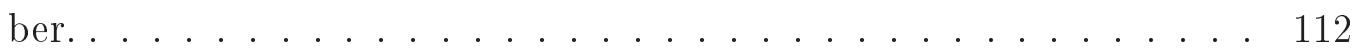

4.3 Performance of $\psi^{\mathrm{CARL}}$ and $\psi^{\mathrm{DARL}}$ in finding the correct channel access

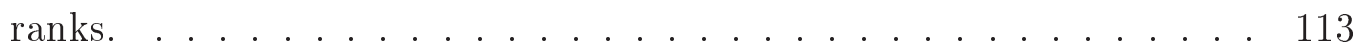

5.1 Statistic calculation in Algorithm 5.1 . . . . . . . . . . . . 122

5.2 Statistic calculation in Algorithm 5.2 . . . . . . . . . . . . 123

5.3 Channel re-ranking example for a SU $i \ldots \ldots$. . . . . . . . . . 127 
THIS PAGE INTENTIONALLY LEFT BLANK 


\section{List of Symbols}

A

$\mathcal{R}$

$\mathcal{R}^{c}$

$N$

$\delta$

$p_{h}$

$\gamma$

$M$

$C_{j}$

$\mathcal{B}$

$\mathcal{N}\left(C_{j}\right)$

$f_{C_{j}}$

$\lambda$

$\mathcal{D}$

$b$

$B_{x}(\delta) \quad$ disk of radius $\delta$ centered at $x$

no-talk region of $\mathrm{PU}$

broadcast range of a $\mathrm{SU}$

volume of the region $\mathcal{D}$ region of interest containing the PU and all SUs

complement of $\mathcal{R}$ in the region of interest $A$

number of SUs in region of interest $A$ in Chapter 3

probability of a SU to become a cluster head

threshold to determine if a cluster is a boundary cluster

number of boundary clusters in Chapter 3

the $j$ th boundary cluster, $j=1, \ldots, M$

set of boundary clusters $\left\{C_{1}, \ldots, C_{M}\right\}$

set of neighboring clusters of $C_{j}$

local boundary estimation function of cluster $C_{j}$

rate of SU location Poisson point process

approximate region in $A$ containing all boundary clusters 


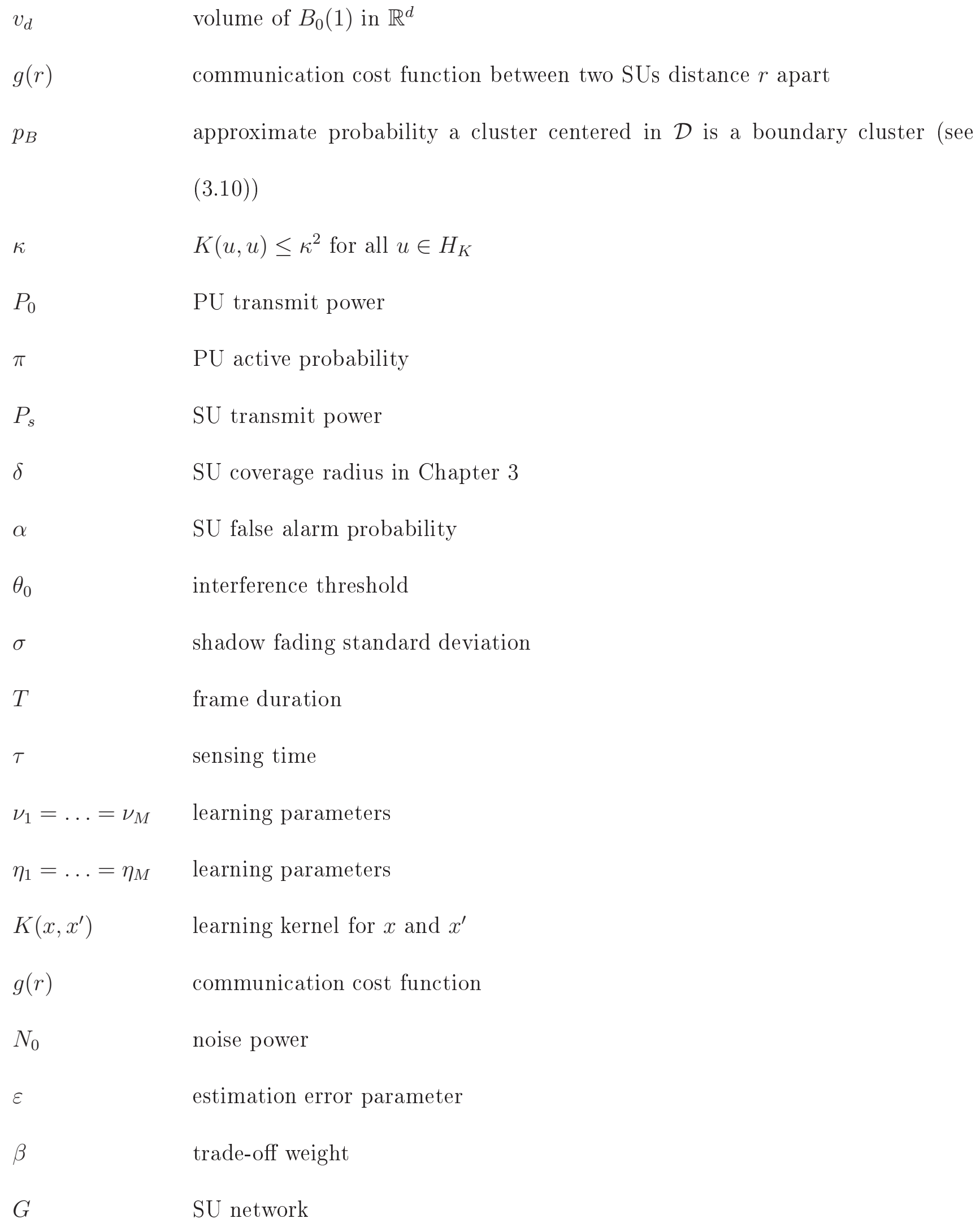




\begin{tabular}{|c|c|}
\hline $\mathcal{N}$ & set of channels \\
\hline $\mathcal{N}^{+}$ & set of channels $\mathcal{N}$ augmented with the null channels such that $\left|\mathcal{N}^{+}\right| \geq M$ \\
\hline$\mu_{j}$ & idle probability of channel $j$ \\
\hline $\mathcal{M}_{i}$ & set of neighboring SUs of the SU $i$ \\
\hline$\left|\mathcal{M}_{i}\right|$ & number of neighboring SUs of the SU $i$ \\
\hline$n$ & time slot \\
\hline$X_{i, j}(n)$ & sensing decision of SU $i$ for channel $j$ in time slot $n$ \\
\hline$Y_{i, j}(n)$ & reward of SU $i$ from accessing channel $j$ in time slot $n$ \\
\hline$R(n, \psi)$ & regret of policy $\psi$ until time slot $n$ \\
\hline$r_{i}(n)$ & channel access rank of SU $i$ in time slot $n$ \\
\hline$\rho_{i}(n)$ & channel identity sensed by $\mathrm{SU} i$ in time slot $n$ \\
\hline P0 & channel allocation optimization problem \\
\hline$t_{k}$ & $k$-th time instance to solve $(\widehat{\mathrm{P} 0})$ \\
\hline$\xi(n)$ & number of time instances to solve $(\widehat{\mathrm{P} 0})$ within $n$ time slots \\
\hline$l_{k}$ & number of time slots between $t_{k+1}$ and $t_{k}$ \\
\hline$\gamma$ & parameter in channel learning algorithm \\
\hline$\delta$ & parameter in channel learning algorithm in Chapter 4 and Chapter 5 \\
\hline$\epsilon_{n}$ & probability to sense a channel $j \in \mathcal{N}$ based on $\gamma$ and $\delta$ in time slot $n$ \\
\hline$\chi(G)$ & chromatic number of the graph $G$ \\
\hline$I_{j}$ & maximal independent set $j$ of the graph $G$ \\
\hline$d(i)$ & degree of $\mathrm{SU} i$ : the number of neighboring $\mathrm{SUs}$ for $\mathrm{SU} i$ in the network graph \\
\hline
\end{tabular}


$u(i) \quad$ palette of forbidden colors for $\mathrm{SU} i$

$\triangle(G) \quad$ maximum vertex degree 


\section{Chapter 1}

\section{Introduction}

\subsection{Motivation and Objective}

Radio spectrum refers to the frequencies that are used by various radio-communication services. As a natural resource, the radio spectrum is usually regulated by government agencies, such as the Federal Communications Commission (FCC) in the United States (US), the Office of Communications (Ofcom) in the United Kingdom (UK) and Infocomm Development Authority (IDA) in Singapore. Currently, most countries follow a static spectrum allocation policy $[1,2]$ with little or no sharing of the spectrum among services. With the increasing popularity of mobile devices and need for high speed data transmission, static spectrum allocation policies can no longer satisfy all demands for spectrum. Studies have shown that at any given time, and at any given geographical location, $15 \%-85 \%$ of the spectrum is unused [3].

A cognitive radio (CR) network refers to an intelligent wireless communication system that can learn from and adapt to its environment and internal states by making 
corresponding modifications to certain parameters (e.g., carrier frequency, bandwidth, transmit power, etc.) [4]. It improves radio spectrum utilization by permitting unlicensed secondary users (SUs) to access the same spectrum when the licensed primary users (PUs) are not using it, or when SU transmissions do not interfere significantly with the PUs. For example, in the US, it has been found that television (TV) and digital video broadcast (DVB) white spaces can be used for unlicensed wireless broadband access [5].

In cognitive radio networks (CRNs), opportunistic spectrum access (OSA) increases spectrum utilization by allowing unlicensed secondary users (SUs) to identify and exploit the unused spectrum owned by primary users (PUs) opportunistically while limiting the interference to PUs below a predefined threshold. Due to the requirements of more spectrum users and higher transmission rate nowadays, effective identification of spectrum access opportunities is one of the major tasks in designing cognitive radio systems. Thus, various spectrum sensing methods have been proposed for OSA, including centralized [6-9], distributed [10] and relay-assisted cooperative detection schemes [11]. However, many works require the central authority to track spectrum usage of all PUs and SUs, which results in high processing complexity for the central authority [7-9]. Other works need SUs to perform spectrum sensing individually $[9,12]$, which suffer from low spectrum sensing accuracy, and may not always be able to detect the presence of the "hidden PU". Also a lot of works in CR spectrum sensing are concerned with opportunistically exploiting spectrum holes as a result of the PU's temporal usage diversity $[13,14]$ and did not consider the joint temporal-spatial usage diversity of the PU. Moreover, there are lots of works that 
have investigated OSA at the medium access control (MAC) layer [15-17]. However, most of these works require some information about PUs and also did not consider spatial spectrum reuse.

In this thesis, we propose and analyze learning methods for cognitive radio networks to exploit opportunistic temporal-spatial spectrum access, without the secondary users having a priori knowledge of the primary user's coverage region and channel idle probabilities.

We firstly consider the joint temporal-spatial usage diversity of the PU for OSA by letting the PU fix an interference temperature limit that allows for interference from SUs in its licensed spectrum below a threshold [18]. This translates to an interference coverage region around the $\mathrm{PU}$, in which SUs opportunistically transmit only if the $\mathrm{PU}$ is not transmitting. SUs outside of this coverage region can transmit regardless of whether the PU is active or not. Our main goal is to develop a distributed learning algorithm that allows SUs to cooperatively determine the boundary of this coverage region in order to improve spectrum efficiency.

Then, within the no-talk region of the PU, SUs who do not interfere with each other can make use of the same PU channel. We investigate temporal-spatial spectrum reuse within the no-talk region of a $\mathrm{CRN}$, where $\mathrm{PU}$ channel idle probabilities are unknown to the SUs. We then formulate and study a multi-user multi-armed bandit (MAB) problem that exploits the temporal-spatial OSA of PU channels for these SUs located inside the region. To the best of our knowledge, this is the first work to consider temporal-spatial spectrum reuse in a MAB formulation.

Moreover, in recent years, collaborative MAB have been widely investigated with 
the aim of achieving the speed-up in learning performance in many computationally intensive, large-scale applications. Lots of collaborative MAB algorithms have been proposed and have helped us obtain rank results for large-scale search engines [19,20], choose to display relevant advertisements on web sites for maximizing their clickthrough rate [21-23] and accelerate the optimization process [24]. We investigate the collaborative MAB problem in CRNs for spectrum reuse, in which SUs could exchange some information with their neighboring SUs in the learning process and simulations show that the learning performance has significantly improved. This implies that our proposed polices could be potentially implemented in large-scale CRNs.

\subsection{Contributions of the Thesis}

In this thesis, we investigate the problem of identifying spectrum access opportunities by SUs in CRNs. We propose several learning methods for temporal-spatial OSA. We give an outline of our contributions in the following sections.

\subsubsection{Distributed Boundary Learning For Spectrum Sensing}

We firstly consider the cooperative estimation of the PU's no-talk region by exploiting local communications amongst SUs. Our main contributions are as follows:

1. We propose a distributed boundary estimation method based on the distributed learning framework of [25], and with additional smoothness constraints. Sensors outside the estimated no-talk region are allowed to transmit even if the PU is transmitting. 
2. We provide approximate theoretical bounds for the communication cost incurred by our proposed method and the expected estimation error, so that the approximate optimal SU density can be inferred. This is useful for randomly allocating SUs to estimate the no-talk regions of multiple PUs transmitting over different frequency bands. We note that our theoretical performance analysis is not considered in [25], and to the best of our knowledge, is new.

3. We derive order bounds for the setup complexity of our proposed method, and expressions for the throughput achievable by the PU and SUs.

4. Simulations suggest that our proposed boundary estimation algorithm has better trade-offs in the throughput and setup communication cost than various other boundary estimation algorithms in the literature, and is more robust to SU sensing errors except when compared to the centralized least squares SVM (LS-SVM) method, which however incurs a much higher communication cost.

Our method allows better spatial usage of the spectrum and improves the overall system throughput, albeit at the cost of estimating the boundary. For a stationary $\mathrm{PU}$, this is a fixed cost that does not contribute significantly to the overall operational energy cost. An example is the use of CR systems in the Internet-of-Things framework [26], where devices like electrical appliances are fixed and CRs in the devices allow opportunistic use of the cluttered spectrum. 


\subsubsection{Channel Allocation and Learning For Temporal-Spatial Spectrum Reuse}

Then, we investigate the temporal-spatial OSA problem in a CRN within the no-talk region of the $\mathrm{PU}$, where $\mathrm{PU}$ channel idle probabilities are unknown to the SUs. We say that a SU has been allocated a channel access rank $k$ if it is assigned to use only the $k$-th best channel (in terms of idle probability). Our main contributions are as follows:

1. We propose a centralized policy that uses a central processor to optimize the channel access ranks of the SUs at exponentially increasing time intervals, based on the idle probability estimates of an arbitrary SU. SUs then perform a local random $\epsilon$-greedy channel learning algorithm. We call this the Centralized Channel Allocation (CCA) policy.

2. To overcome the requirement for a central processor, we propose a distributed three-stage policy to enable SUs to learn their channel access ranks and the channel idle probabilities. In our proposed policy, we adopt a distributed synchronous greedy graph coloring algorithm [27] to cluster SUs into channel access groupings, and a distributed average consensus algorithm [28] to learn the sizes of the groups, with SUs belonging to a larger group being assigned a smaller access rank. Then, each SU independently learns the PU channel statistics using an $\epsilon$-greedy policy [29] based on its assigned access rank. The outcome of our proposed policy is a channel allocation for all the SUs. We call this the Collaborative Access Ranking and Learning (CARL) policy. 
3. To avoid the need for SU synchronization and the overhead incurred by the CARL policy, we also propose a distributed channel learning and allocation policy that integrates the first two stages of CARL into the $\epsilon$-greedy learning process. We call this the Distributed Access Rank Learning (DARL) policy.

4. We provide theoretical bounds on the regrets achieved by our proposed CCA, CARL and DARL policies, the random access policy [30], the time-division fair sharing (TDFS) policy [31], and the adaptive randomization policy [30]. We show the CCA policy has logarithmic regret, while all other policies have linear regret. We provide simulation results to verify the performance of our proposed CCA, CARL and DARL policies. Our simulation results suggest that CCA, CARL and DARL perform significantly better in terms of average regret than the random access policy, the TDFS policy and the adaptive randomization policy.

\subsubsection{Collaborative Learning For Temporal-Spatial Spectrum Reuse}

To improve the speed in learning the channel statistics, we investigate the collaborative MAB problem [32] and propose three collaborative channel learning policies in Chapter 5 to identify temporal-spatial spectrum access opportunities via information exchange among neighboring SUs. Simulation results show that these collaborative learning polices could largely improve the learning performance. Our main contributions are as follows: 
1. We propose a collaborative learning policy in which each SU will perform an average calculation on the sensing observations of its neighboring SUs for their selected channels. SUs then perform a local random $\epsilon$-greedy channel learning algorithm [29] after combining the observations of their neighboring SUs with its own sensing observation. We call this the Collaborative Average Observation Channel Learning (CAOCL) policy.

2. To avoid the need for SU to broadcast its sensing observation, we propose a collaborative learning policy that let the SU perform a weighted consensus [28] on the empirical idle probability estimates of its neighboring SUs for their selected channels. Then, SUs similarly perform a local random $\epsilon$-greedy channel learning algorithm [29] using the updated estimates of channel idle probabilities. We call this the Collaborative Average Mean Channel Learning (CAMCL) policy.

3. For privacy and security considerations, we design a collaborative learning policy without utilizing the sensing observations or the empirical idle probability estimates from their neighboring SUs. Each SU learns the correct channel ranks by performing a majority rule on the estimated channel ranks obtained from its neighboring SUs. SUs then apply a local random $\epsilon$-greedy channel learning algorithm [29] based on the updated channel ranks. We call this Collaborative Channel Rank Learning (CCRL) policy.

4. We provide simulation results to show our proposed polices CAOCL, CAMCL and CCRL all perform better than the CARL policy in terms of average regret.

Parts of this thesis have appeared in [33-37]. In [33,34], we studied the problem 
of learning the boundary of the no-talk region of a $\mathrm{PU}$ for temporal-spatial OSA and proposed a distributed boundary estimation algorithm which can collaboratively determine the boundary of PU's no-talk region. In [35-37], we investigated a multiuser multi-armed bandit problem for temporal-spatial spectrum reuse within the notalk region of the PU and several channel access rank allocation and channel statistics learning policies were proposed, which can largely increase the total network reward.

\subsection{Organization of the Thesis}

In Chapter 2, we provide an overview of the existing studies for opportunistic spectrum access on spectrum sensing, boundary estimation, multi-armed bandit and collaborative learning. In Chapter 3, we introduce our system model and the problem formulation for distributed boundary estimation of the no-talk region of the PU. We propose a distributed boundary estimation algorithm for learning the boundary of the no-talk region of the PU. We analyze the trade-offs between communication cost and estimation error of our boundary estimation method for a Poisson field of SUs, and determine its setup complexity and throughput. Then, simulation results are provided. In Chapter 4, to maximize the spectrum usage within the no-talk region of the PU, we formulate a multi-user multi-armed bandit (MAB) problem for temporalspatial OSA. We propose a centralized channel allocation policy that uses a central processor to determine the SUs' channel access ranks. Moreover, we propose two distributed policies that enable SUs to find their channel access ranks and independently learn the channel statistics. Furthermore, we give simulation results for verifying their 
performance. In Chapter 5, we consider the collaborative MAB problem and propose three collaborative learning policies. Simulation results are also provided. Finally, we conclude this thesis and discuss some future research directions in Chapter 6 . 


\section{Chapter 2}

\section{Related Works}

Static spectrum allocation has been shown to result in spectrum under-utilization [38].

In cognitive radio networks (CRNs), opportunistic spectrum access (OSA) alleviates the problem by allowing unlicensed secondary users (SUs) to identify and exploit the unused spectrum owned by primary users (PUs) opportunistically while limiting the interference to PUs below a predefined threshold. OSA finds application as the underlying communication paradigm in sensor networks and the Internet of Things [39-43].

OSA enables SUs to dynamically search for spectrum opportunities, e.g. spectrum holes or white spaces in temporal, spatial and frequency domains and it has been extensively studied at the physical (PHY) and medium access control (MAC) layers. Various temporal [6,44-46], spatial [47-51], or temporal-spatial [34, 52, 53] spectrum-sensing algorithms have been proposed to detect PUs and identify spectrum opportunities temporally and spatially with acceptable interference to PUs. We firstly describe some spectrum-sensing related works in Section 2.1. 
However, the experimental results in [54] show that the traditional PHY layer model is not suitable for solving practical communication issues, e.g. multiple access control. Therefore, OSA at the medium access control (MAC) layer has been widely studied to exploit spectrum access and sharing opportunities for SUs based on their spectrum-sensing results $[15,16]$. Many MAC protocols use a centralized coordinator (e.g., a base station or a spectrum allocation server) or a dedicated communication channel to control spectrum access and allocation [55]. Such methods suffer several disadvantages, including the selection of a site for the coordinator, the vulnerability of the CRN to failure of the coordinator, and the requirement of frequent communications in a dedicated control channel. Moreover, these methods do not consider the interference between neighboring SUs if they transmit in the same channel simultaneously.

To study the interactions among SUs in a distributed manner, game theory is widely used to design efficient distributed OSA mechanisms [17, 56, 57]. However, most of these works do not exploit spatial spectrum reuse and assume that each SU interferes with every other $\mathrm{SU}$ in the CRN. To allow for spatial spectrum sharing amongst the SUs, graphical game algorithms have formulated spatial reuse of the spectrum as a local bargaining process [58], a local minority game [59], a spatial congestion game [60] or a MAC-layer interference minimization game [61]. However, these works assume that some information about PUs like the location or channel usage of PUs is known by all the SUs.

Multi-armed bandit (MAB) techniques $[30,31,57,62]$ have been applied to exploit spectrum access and sharing opportunities for SUs based on their spectrum- 
sensing results when PU channel information is unknown. Since we formulate and study a multi-user multi-armed bandit (MAB) problem in Chapter 4 that exploits the temporal-spatial spectrum access opportunities without knowing any information about PU, we also summarize some related works that used MAB approaches in Section 2.2 to have a basic understanding of this area.

Recently, there has been an increasing interest in designing collaborative MAB polices for large-scale networks. Users can share some information and collaboratively achieve their common goal in the learning process. By adopting the idea of collaborative learning, the learning performance can significantly speed up with some communication cost $[32,63]$. In Section 2.3, we introduce some works that studied the collaborative MAB problem.

\subsection{Spectrum Sensing and Boundary Estimation}

PU detection schemes in which a central authority (e.g., a base station or dedicated coordinator) tracks the spectral usage and assigns channels to SUs have been proposed [64]. These methods are typically based on energy detection [7,8], matched filtering [9] and feature detection [12] techniques. However, requiring the central authority to track spectrum usage of all PUs and SUs results in high processing complexity for the central authority. Another approach is for SUs to perform spectrum sensing individually $[9,12]$. Both these approaches suffer from low spectrum sensing accuracy, and may not always be able to detect the presence of the PU if there are interference walls between the PU and the sensor, resulting in a "hidden PU" [65]. 
In order to circumvent these problems, cooperative detection schemes, including centralized [6,66-68], distributed [10] and relay-assisted cooperation [11,69,70] have been proposed. In centralized cooperative sensing, each SU performs its own local sensing and reports its sensing decision to a fusion center. The fusion center combines the local decisions to determine the presence of the PU. Such cooperation has been shown to improve the detection probability while keeping the false alarm rate the same [6]. Unlike centralized cooperative sensing, distributed cooperative sensing does not rely on a fusion center to make the final decision. Each SU receives the sensing decisions from neighboring SUs, combines that with its own observations, and decides whether the PU is active or not. The process is repeated until all the SUs reach a common decision. In the relay-assisted cooperative sensing method, a SU who owns a weak sensing channel but a strong report channel will work together with a SU who has a strong sensing channel and a weak report channel so that the maximum diversity gain becomes achievable for each user.

Most works in CR spectrum sensing are concerned with opportunistically exploiting spectrum holes as a result of the PU's temporal usage diversity [13,14], while fewer works studied the problem of collaboratively identifying spatial spectrum access opportunities. In our work, we consider the joint temporal-spatial usage diversity of the PU for OSA by letting the PU fix an interference temperature limit that allows for interference from SUs in its licensed spectrum below a threshold [18]. This translates to an interference coverage region around the PU, in which SUs opportunistically transmit only if the PU is not transmitting. SUs outside of this coverage region can transmit regardless of whether the PU is active or not. In [71-73], different spectrum 
sharing regions, including a primary exclusive region and the no-talk region, are defined. However, all these works assume that the propagation path loss between the PU and SUs are isotropic, and all regions are assumed to be circular. Bounds on the radius of each region are given based on interference and outage considerations, which are characterized in terms of propagation parameters like path loss exponents. In practice, the propagation environment may be very difficult to model quantitatively, and the no-talk region is unlikely to be circular. Therefore, in this work, we develop a boundary estimation method for learning the boundary of PU's no-talk region without relying on extensive assumptions about the shape of the region.

Boundary estimation is widely used in different sensor networking applications [74], and has been extensively studied [75-80]. Methods based on node degrees [77], connectivity information [79], and topology information [81] have been proposed to estimate the coverage region of a sensor network. Although the definition of a no-talk region was comprehensively addressed in [7], little work has been done to estimate the boundary of this region. A classifier-based cooperative boundary detection algorithm for estimating the no-talk region using support vector machines (SVM) [82] has been proposed in [80]. A computational geometry method based on convex hulls has also been utilized for boundary estimation in [80]. All these works however assume that sensors send their local information to a fusion center, and boundary estimation is performed at the fusion center. In a cognitive radio network, constraints on energy and bandwidth usually restrict SUs from communicating with a single fusion center effectively. The fusion center requires significantly higher processing power than a normal sensor. Moreover, a single fusion center is a point of failure for the whole 
network. Localized edge detection algorithms based on statistical, image processing and classification methods have been proposed in [75] to allow a sensor to locally decide whether or not it is located on or near a boundary. A distributed Bayesian algorithm has also been proposed to determine event regions $[83,84]$. These methods however do not make use of cooperation between sensors. A hierarchical tree-based estimation method using recursive dyadic partitioning [74] and a dynamic boundary tracking algorithm that combines spatial and temporal estimation techniques [85] have been proposed for boundary estimation in ad-hoc networks. However, this method is again centralized, and does not consider the smoothness of the estimated boundary.

In our work, we consider the cooperative boundary learning problem, which aims to estimate the no-talk region of the PU for OSA by applying a distributed learning framework and exploiting local communications amongst SUs. The smoothness of the estimated boundary is also considered.

\subsection{Multi-armed Bandit}

The multi-armed bandit (MAB) problem is a classical paradigm in machine learning field in which at each time slot, an online algorithm needs to select one of arms (strategies) for maximizing the total rewards over all the time slots. Many real-world applications have been modeled as different kinds of multi-armed bandit problems, ranging from the treatment selection in clinic trails [86], Internet advertising optimization [23, 87], packet routing [88], on-line auctions [89] and target tracking in multi-agent systems [90-92], etc. The key issue in solving multi-armed bandit prob- 
lems is to balance the trade-off between acquiring new knowledge from using a underexplored strategy and capitalizing on the available information from exploiting those well known strategies.

The MAB problem has been extensively studied and lots of policies have been proposed in the literature $[23,29,93-96]$. Lay and Robbins have developed an indextype policy for restrictive and specific families of reward distributions (indexed by a single real parameter) by using sample mean statistic [93]. Later, several index-type polices have been introduced in [29], which could achieve order optimality for all reward distributions with bounded support. Recent works focus on designing polices under specific situations, including MAB in a changing environment [94,97], MAB with contextual information [23,96], exploration-exploitation learning with limited resources [98, 99], risk-reward tradeoff in MAB [95], etc.

Multi-armed bandit (MAB) techniques could be applied for OSA when PU channel information is unknown. A no-regret learning method was proposed in [57], assuming that the channel selection of each SU is known among all SUs. Accordingly, the learning problem could be simplified as a single-user MAB problem. Several distributed multi-user MAB policies have also been proposed when the reward of each $\mathrm{SU}$ is not observable by other SUs. Each SU needs to sense and access channels by learning the channel states independently. In [31], all SUs are assumed to interfere with one another, and a time-division fair share (TDFS) policy was used to orthogonally divide SUs temporally. The TDFS policy was shown to achieve logarithmic regret under spectrum temporal reuse. Instead of partitioning SUs into different time slots, a distributed channel access policy has been proposed in [30] to incorporate 
adaptive randomization to subdivide SUs into different channels. The total regret is also order-optimal logarithmic. The work in [100] considers the setting where SUs have prioritized rankings and proposed a distributed policy based on the well-known UCB1 policy [29], which yields a uniformly logarithmic regret over time. In [62], the same channel yield different rewards for different SUs. By embedding a bipartite matching algorithm, an on-line index-based distributed learning policy was proposed to achieve order $\log ^{2} n$ regret over time horizon $n$. All these methods assume that all SUs interfere with each other if they use the same channel, and spatial reuse of channels was not addressed.

In our work, within the no-talk region of the PU, we investigate temporal-spatial OSA in CRNs using a multi-user MAB learning approach when SUs have no priori knowledge of the PU's channel idle probabilities. We also propose three polices that can find an optimal channel allocation and learn the statistics of the channels for improving spectrum utilization in CRNs.

\subsection{Collaborative Learning}

Intuitively, users within a community could leverage capacity by sharing observations to effectively achieve their common objective. This allows them to learn from others on selection of a good strategy and avoid the unnecessary explorations of bad strategies. Many real-world applications have adopted the collaboration learning idea when implementing their core algorithms, for example, collaborative filtering in

recommendation systems [101-103], web page retrieval for search engines using the 
PageRank algorithm [104] and reputation evaluation systems in the large e-commerce platforms [105], e.g., Taobao and eBay, etc. It is observed that lots of large-scale applications have greatly benefited from applying the collaborative methodology with more accurate ranking results for search engines, a higher click-through rate for recommendation systems and a faster optimization process, etc.

Moreover, in the context of applying OSA in a CRN, the learning workload will be high if a large amount of channel statistics need to be learnt. And the learning speed will be slow so that the learning accuracy could not be guaranteed in a considerable computation time. By combining the information from various SUs in the learning process, the spectrum learning performance will be improved, especially in the computationally intensive and large-scale networks. Some collaborative methods inspired by the new technologies in recommendation systems have been proposed in $[106,107]$ for OSA. These schemes required SUs to send their channel recommendations to neighboring SUs at the end of each time slot and enabled each SU to make use of the correlations in time and space domains for making more informed decisions. However, they all assume that some information about PUs is known.

Comparing with the literature on non-collaborative MAB, collaborative MAB problem is not well studied. There are some policies that have been proposed for the scenarios in which there are some competitions among users [108,109]. The work in [108] generalized the adversarial multi-armed bandit problem [110] and assumed that there is a set of dishonest users in the collaborative MAB learning process, who will manipulate their actions in a Byzantine manner to achieve their collectivized goals. An algorithm named TrustFilter was proposed to evaluate the reputation of 
each user as well as learn to select its optimal resource. In [109], two policies were proposed to identify the best arm for each user who competed with each other on arm-pulls resources. In contrast, there are some recent works which investigated noncompetitive information sharing schemas among users [32,63]. Three collaborative best-arm identification algorithms have been introduced in [32] which enable users to achieve speed-up in the learning process with rounds of inter-user communications. The work in [63] studied the online distributed expert problem and performed analysis on minimizing the regret while keeping the communication cost to a minimum.

In our work, we study a collaborative learning problem without competitions among SUs. We make an extension from a proposed distributed policy in Chapter 4 by allowing SUs to collaboratively learn the channel statistics and spatially reuse the channels. This discussion will facilitate the implementation of our proposed policies in the large-scale CRNs. We also note that our problem is somewhat similar to the problem of batch learning $[111,112]$ in terms of motivation, which is to accelerate the learning speed after obtaining a set of observations. However, our MAB problem is online in nature and observations are collected in sequence of rounds. Therefore, most batch learning techniques [113] can not directly apply to our problem. 


\section{Chapter 3}

\section{Distributed Boundary Learning for}

\section{Spectrum Sensing}

In this chapter, we consider the temporal-spatial usage diversity of the PU for OSA by letting the PU fix an interference temperature limit that allows for interference from SUs in its licensed spectrum below a threshold $[7,18]$. This translates to a notalk region around the $\mathrm{PU}$, in which SUs opportunistically transmit only if the $\mathrm{PU}$ is not transmitting $[7,71]$. SUs outside of this no-talk region can transmit regardless of whether the PU is active or not. We aim to develop a distributed learning algorithm that allows SUs to cooperatively determine the boundary of the no-talk region. The proposed method is based on the distributed learning framework of [25], with additional smoothness constraints. Moreover, approximate theoretical bounds are provided for the communication cost and the expected estimation error incurred by our proposed method, which is not considered in [25], and to the best of our knowledge, is new. 
The rest of this chapter is organized as follows. In Section 3.1, we introduce our system model and problem formulation. In Section 3.2, we propose a distributed boundary estimation algorithm for learning the boundary of the no-talk region $\mathcal{R}$. In Section 3.3, we analyze the trade-offs between communication cost and estimation error of our boundary estimation method for a Poisson field of SUs, and determine its setup complexity and throughput. Simulation results are provided in Section 3.4. Finally, we conclude in Section 3.5.

\subsection{Problem Formulation}

Suppose that there is one PU and $N$ SUs in a bounded region $A \subset \mathbb{R}^{d} .{ }^{1}$ We say that the $\mathrm{PU}$ is active if it is transmitting in its licensed spectrum. Suppose that the PU is located at $x_{0}$. To simplify the analysis, we assume that all wireless channels are additive white Gaussian noise (AWGN) channels. We define the no-talk region [7] of the PU to be the set $\mathcal{R}=\left\{x \in \mathbb{R}^{d}: P_{0}-L\left(x, x_{0}\right)>\theta_{0}\right\}$, where $P_{0}$ is the transmit power of the $\mathrm{PU}, L\left(x, x_{0}\right)$ is the average propagation loss function between the $\mathrm{PU}$ and a SU located at $x$, and $\theta_{0}$ is a fixed threshold. The average propagation loss can be modeled as $L\left(x, x_{0}\right)=l\left(\left\|x-x_{0}\right\|\right)+S\left(x, x_{0}\right)+F\left(x, x_{0}\right),{ }^{2}$ where $l\left(\left\|x-x_{0}\right\|\right)$ is the power attenuation due to the distance $\left\|x-x_{0}\right\|$ between a SU at location $x$ and the PU at location $x_{0}, S\left(x, x_{0}\right)$ represents the average shadowing effect, and $F\left(x, x_{0}\right)$ is the average power loss due to multipath fading. We suppose that the PU can

\footnotetext{
${ }^{1}$ In most applications, $d=2$ or 3 , which corresponds to SUs scattered over a geographical region or in a building respectively.

${ }^{2}$ All power quantities are expressed in $\mathrm{dB}$.
} 
tolerate an average interference below the fixed threshold $\theta_{0}$ so that SUs outside of $\mathcal{R}$ can utilize the PU spectrum regardless of whether it is active or not. SUs within the no-talk region $\mathcal{R}$ are required to refrain from using the PU spectrum if the PU is transmitting. Note that the threshold $\theta_{0}$ is chosen to include a safety margin or budget for the propagation loss due to shadowing and fading, and other parameters like the average density of SUs. The reader is referred to [7] for a detailed discussion of the different considerations involved in defining the no-talk region of a PU.

In this thesis, we aim to estimate the no-talk region $\mathcal{R}$, or equivalently the boundary of $\mathcal{R}$, in order to facilitate spatial spectrum sharing between the PU and SUs. The average propagation loss $L\left(x, x_{0}\right)$ for a $\mathrm{SU}$ at position $x$ depends on various factors including the terrain, the type and number of reflectors and attenuators between the PU and SU, and other ambient environmental factors. The propagation loss function is thus difficult to determine to good accuracy in practice, and therefore we assume that $L\left(x, x_{0}\right)$ is unknown, and adopt a learning approach to estimate the region $\mathcal{R}$ solely based on the received power at the SUs. We make the following assumptions.

\section{Assumption 3.1.}

(a) Communications by SUs are over relatively shorter distances than the PU, and hence the transmit power of each $\mathrm{SU}$ is at most $P_{0}$. Multiple SUs can share the PU's spectrum spatially (see Figure $3-1){ }^{3}$

(b) The region $\mathcal{R}$ is compact, and has a smooth ${ }^{4}$ boundary.

\footnotetext{
${ }^{3}$ Various spectrum sharing schemes have been described in [114].

${ }^{4}$ Formally, this means that the boundary is parameterizable and differentiable in that parameter.
} 
(c) Time can be discretized into intervals and the PU is active in each interval with known probability $\pi \in[0,1]$, independently across intervals. ${ }^{5}$

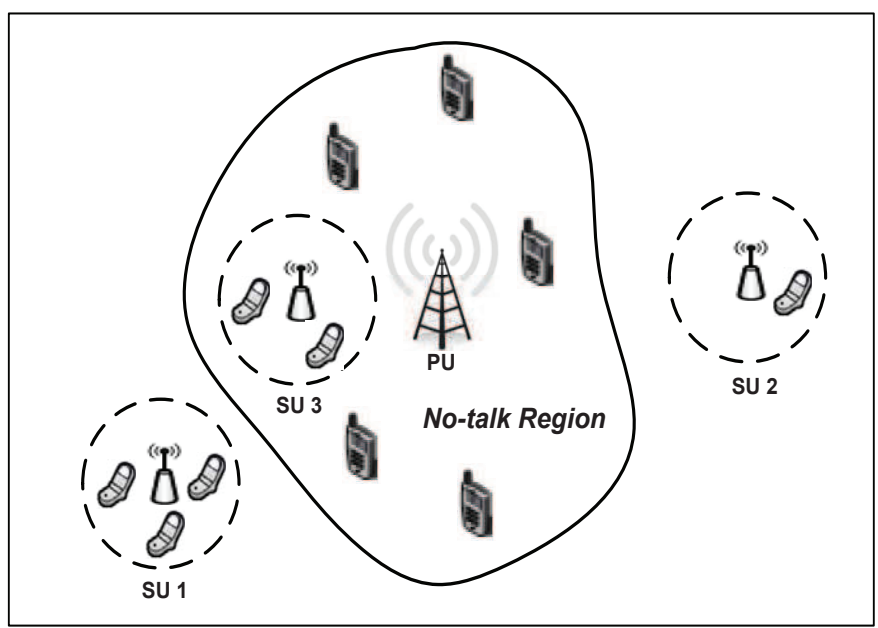

Figure 3-1: Spatial spectrum sharing between PU and multiple SUs. SU 1 and 2 can use the licensed spectrum of the PU without spectrum sensing. SU 3 can only utilize the spectrum when the $\mathrm{PU}$ is inactive.

We let each SU sample the PU licensed spectrum over a sufficiently long calibration phase in order to perform boundary estimation of $\mathcal{R}$. We assume that $\mathcal{R}$ has a smooth boundary in Assumption 3.1(b) to avoid the case where a temporary degradation in the channel between the PU and a SU during the calibration phase may incur a large estimation error. This assumption is also valid in most practical situations, except when there are strong attenuators close to the boundary, in which case our solution leads to an estimated no-talk region larger than the actual one. In our problem formulation (cf. Section 3.2.2), we will not impose Assumption 3.1(b) strictly, but rather adopt a simpler constraint to approximate it. In addition, for practicality, we require that the estimation algorithm is distributed, with each SU having access only

\footnotetext{
${ }^{5}$ Although we restrict our analysis to the case where the PU active probability $\pi$ is the same across intervals, our analysis can be easily generalized to the case where each interval has a different PU active probability.
} 
to local information (its own observations and information from its neighbors).

Suppose that SU $i$ is located at $x_{i}$, for $i=1, \ldots, N$, and suppose that the calibration period for $\mathrm{SU} i$ is divided into $J$ observation intervals, where the PU is active with probability $\pi$ during each interval independently (cf. Assumption 3.1(c)). Let $Y_{i}[j]$ be the signal sample obtained by $\mathrm{SU} i$ in interval $j$, where $j=1, \ldots, J$. If the $\mathrm{PU}$ is inactive during interval $j$, we have

$$
Y_{i}[j]=W_{i}[j]
$$

where the noise variables $W_{i}[j]$ are independent zero mean Gaussian random variables with variance $\sigma_{W}^{2}$. If the $\mathrm{PU}$ is active during interval $j$, we have

$$
Y_{i}[j]=X_{i}[j]+W_{i}[j]
$$

where $X_{i}[j]$ is the PU signal, which is approximated to be a Gaussian random variable with zero mean, and independent of $W_{i}[j]$ for simplifying the analysis. The variance or power of $X_{i}[j]$ is then given by $\mu_{i}=P_{0}-L\left(x_{i}, x_{0}\right)$. Assuming the PU signal $X_{i}[j]$ to be a Gaussian random variable is also adopted by many related works $[6,7,69,71]$.

Let $\mathcal{H}_{0}: \mu_{i} \leq \theta_{0}$ and $\mathcal{H}_{1}: \mu_{i}>\theta_{0}$ be the hypotheses that $\mathrm{SU} i$ is outside and within the no-talk region $\mathcal{R}$, respectively. For the sake of generality, we assume that the PU signal modulation scheme is unknown to the SUs. Therefore, SUs are constrained to use energy detection methods $[7,9]$ in order to perform the hypothesis test. For this purpose, $\mathrm{SU} i$ forms the test statistic $T_{i}=\frac{1}{J} \sum_{j=1}^{J}\left|Y_{i}[j]\right|^{2}$ and uses the following 
threshold rule to determine the hypothesis:

$$
T_{i} \stackrel{\mathcal{H}_{0}}{\stackrel{\mathcal{H}_{1}}{\leq}} \theta,
$$

where $\theta$ is chosen so that the false alarm probability is below a predefined threshold $\alpha \in(0,1)$. Since $\mu_{i}$ is unknown a priori, we need to make further approximations in order to determine $\theta$. The mean of $T_{i}$ is given by

$$
m\left(\mu_{i}\right)=\pi \mu_{i}+\sigma_{W}^{2}
$$

and the variance of $T_{i}$ is derived using

$$
\eta\left(\mu_{i}\right)=\frac{1}{J}\left(\mathbb{E}\left[Y_{i}[j]^{4}\right]-\left(\mathbb{E}\left[Y_{i}[j]^{2}\right]\right)^{2}\right)
$$

With probability $\pi, Y_{i}[j]$ is a Gaussian random variable with zero mean and variance $\mu_{i}+\sigma_{W}^{2}$ and with probability $1-\pi, Y_{i}[j]$ is a Gaussian random variable with zero mean and variance $\sigma_{W}^{2}$, Then, the variance of $T_{i}$ is given by

$$
\begin{aligned}
\eta\left(\mu_{i}\right) & =\frac{1}{J}\left((1-\pi) \mathbb{E}\left[\sigma_{W}^{4}\right]+\pi \mathbb{E}\left[\left(X_{i}[j]+\sigma_{W}\right)^{4}\right]-\left(\mathbb{E}\left[Y_{i}[j]^{2}\right]\right)^{2}\right) \\
& =\frac{1}{J}\left(3(1-\pi) \sigma_{W}^{4}+3 \pi\left(\mu_{i}+\sigma_{W}^{2}\right)^{2}-\left(\pi \mu_{i}+\sigma_{W}^{2}\right)^{2}\right) \\
& =\frac{1}{J}\left(\mu_{i}^{2} \pi(3-\pi)+2 \sigma_{W}^{2}\left(\sigma_{W}^{2}+2 \pi \mu_{i}\right)\right) .
\end{aligned}
$$

Assuming that $J$ is sufficiently large, the central limit theorem [115] allows us to approximate the distribution of $T_{i}$ as a Gaussian distribution with mean $m\left(\mu_{i}\right)$ and 
variance $\eta\left(\mu_{i}\right)$. Since both $m(\cdot)$ and $\eta(\cdot)$ are increasing functions, the threshold $\theta$ can now be chosen to ensure that the false alarm constraint in the worst case situation is satisfied, by setting

$$
\mathcal{Q}\left(\frac{\theta-m\left(\theta_{0}\right)}{\sqrt{\eta\left(\theta_{0}\right)}}\right)=\alpha
$$

where $\mathcal{Q}(\cdot)$ is the complementary cumulative distribution function for the standard normal distribution. We note that the test (3.1) and the choice of $\theta$ do not require knowledge of the PU transmit power $P_{0}$ or the locations of the PU and SUs.

When there are multiple PUs transmitting in the same spectrum, the no-talk region is the union of all PUs' no-talk regions. Suppose there are $N^{\prime}>1$ PUs, and that all PU signals are uncorrelated. Let $Y_{i p}[j]$ be the signal sample received by $\mathrm{SU} i$ from the $\mathrm{PU} p$ in the interval $j$. For a sufficiently large $J$ and $p \neq p^{\prime}$, we have $\frac{1}{J} \sum_{j=1}^{J} Y_{i p}[j] Y_{i p^{\prime}}[j] \approx 0$, and the test statistic $T_{i}=\frac{1}{J} \sum_{j=1}^{J}\left|\sum_{p=1}^{N^{\prime}} Y_{i p}[j]\right|^{2}$ can be approximated as $\frac{1}{J} \sum_{p=1}^{N^{\prime}} \sum_{j=1}^{J}\left|Y_{i p}[j]\right|^{2}$. A threshold for $T_{i}$ similar to that in (3.1) can be found to determine if $\mathrm{SU} i$ is within the no-talk region of at least one PU.

Let $u_{i}$ be the decision of SU $i$ for the test (3.1), where $u_{i}=-1$ if it decides in favor of $\mathcal{H}_{0}$, and $u_{i}=1$ otherwise. Recall that $x_{i}$ is the position of SU $i$, and let $y_{i}=\left(x_{i}, u_{i}\right)$, for $i=1, \ldots, N$. Our aim is to learn a function $f: \mathbb{R}^{d} \mapsto \mathbb{R}$ based on the collection of pairs $y=\left\{y_{i}: i=1, \ldots, N\right\}$, so that $f(x) \geq 0$ or $f(x)<0$ if a SU location $x$ is inside or outside the region $\mathcal{R}$ respectively, and with $\{x: f(x)=0\}$ corresponding to the boundary of $\mathcal{R}$. In the same spirit as statistical learning theory [116], we can regard each $y_{i}$ as being drawn independently from the same joint distribution $p(x, u)$ 
(which is unknown because the path loss $L\left(x, x_{0}\right)$ is unknown). Then, in estimating the boundary of $\mathcal{R}$, we hope to obtain a function $f$ with small generalization error

$$
\mathcal{E}=\mathbb{E}\left[(f(X)-U)^{2}\right],
$$

where $U$ is a random variable representing the decision of a generic $\mathrm{SU}$ and $(X, U)$ has joint distribution $p(x, u)$. If $\mathcal{E}$ is large, the throughput at the PU deteriorates because of interference from SUs that wrongly believe themselves to be in $\mathcal{R}^{c}{ }^{6}$ Therefore, we are interested to study the trade-offs in throughputs with $\mathcal{E}$ and the communication cost of performing the boundary estimation.

In a centralized estimation algorithm, the data $y$ is sent to a fusion center, which trains a global function. Such centralized algorithms suffer from several disadvantages, including the need to select a site for the fusion center, the susceptibility of the whole network to a single point of failure at the fusion center, the need for significant processing power and memory storage at the fusion center, and the use of long range communications as the area $A$ becomes large. In this chapter, we consider distributed algorithms, in which each SU communicates only with neighboring SUs to collaboratively estimate the boundary of $\mathcal{R}$.

For the convenience of the reader, we list some commonly used notations in Table 3.1. Some of these notations have been defined in this section, while the remaining ones will be defined formally in the sequel where they first appear. In addition, we adopt the following definitions. For SU $i$ and cluster $C$, we use $i \in C$ to mean $x_{i} \in C$.

\footnotetext{
${ }^{6}$ We use $\mathcal{R}^{c}=A \backslash \mathcal{R}$ to denote the complement of $\mathcal{R}$ in the region of interest $A$.
} 
The number of SUs in $y$ belonging to $C$ is given by $|C|$. The indicator function $\mathbf{1}_{\{S\}}$ equals to 1 if the statement $S$ is true and 0 otherwise.

Table 3.1: Summary of notations used in Chapter 3

\begin{tabular}{|c|l|}
\hline Symbol & Definition \\
\hline$A$ & region of interest containing the PU and all SUs \\
\hline $\mathcal{R}$ & no-talk region of PU \\
\hline$N$ & number of SUs in region of interest $A$ \\
\hline$\delta$ & broadcast range of a SU \\
\hline$p_{h}$ & probability of a SU to become a cluster head \\
\hline$\gamma$ & threshold to determine if a cluster is a boundary cluster \\
\hline$M$ & number of boundary clusters \\
\hline$C_{j}$ & the $j$ th boundary cluster, $j=1, \ldots, M$ \\
\hline $\mathcal{B}$ & set of boundary clusters $\left\{C_{1}, \ldots, C_{M}\right\}$ \\
\hline $\mathcal{N}\left(C_{j}\right)$ & set of neighboring clusters of $C_{j}$ \\
\hline$f_{C_{j}}$ & local boundary estimation function of cluster $C_{j}$ \\
\hline
\end{tabular}

\subsection{Distributed Boundary Estimation}

In this section, we propose a distributed boundary estimation algorithm that determines the boundary of the set $\mathcal{R}$ based on message passing between SUs. The SUs are grouped into clusters, and most communications are over relatively short ranges within clusters. Each cluster has a SU that serves as the cluster head. The cluster head communicates with SUs inside its cluster, performs most of the necessary computations required for distributed estimation of the boundary, and communicates with other cluster heads. Since the intra-cluster communication cost and the intercluster communication cost will be incurred, cluster heads thus expend more energy than typical SUs inside the cluster. Incentives can be designed to compensate cluster 
heads; an example being given higher priority to access the spectrum. Such incentive mechanisms are out of the scope of our current work, and will not be discussed here.

Our distributed boundary estimation procedure consists of the following steps.

(i) Formation of clusters. Each SU independently nominates itself to be a cluster head with probability $p_{h}$. A cluster head broadcasts a message over a control channel to all SUs within a distance $\delta$ to inform them of their inclusion into the cluster. To avoid collisions amongst cluster heads, a carrier sense multiple access protocol [117] is used. Note that a cluster head can also belong to another cluster, and a SU can belong to multiple clusters.

(ii) Boundary cluster identification. We design a metric to identify those clusters that lie close to the boundary of the set $\mathcal{R}$. We call these the boundary clusters.

(iii) Distributed boundary estimation. Messages are exchanged between members of a boundary cluster and its cluster head. In addition, messages are exchanged between cluster heads of neighboring boundary clusters to collaboratively estimate the boundary of $\mathcal{R}$.

In the following subsections, we describe steps (ii) and (iii) in detail.

\subsubsection{Boundary Cluster Identification}

Let $C$ be a cluster, and $\mathcal{U}^{-}=\frac{1}{|C|} \sum_{i \in C} \mathbf{1}_{\left\{u_{i}=-1\right\}}$ to be the fraction of SUs in cluster $C$ with $u_{i}=-1$. The clusters within $\mathcal{R}$ have a higher probability of $1-\mathcal{U}^{-}$being much larger than $\mathcal{U}^{-}$, while the reverse is true for clusters that are far from the 
PU. To identify those clusters that are close to the boundary of $\mathcal{R}$, we let $\mathcal{S}=$ $\max \left(\mathcal{U}^{-}, 1-\mathcal{U}^{-}\right)$, and say that $C$ is a boundary cluster if and only if $\mathcal{S} \leq \gamma$, where $\gamma$ to be a fixed threshold. If $C$ is not a boundary cluster, the cluster head declares it to be within $\mathcal{R}$ if $\mathcal{U}^{-}<1 / 2$, and outside vice versa. We call those clusters in the former class inside clusters, and those in the latter class outside clusters.

\subsubsection{Distributed Boundary Estimation}

To learn a function $f$ that can be used to determine if a new SU location $x$ (not necessarily belonging to the training data $y$ ) is within or outside $\mathcal{R}$, we consider the following approach. A SU at location $x$ queries its cluster head to check the types of cluster it belongs to (recall that a SU may belong to multiple clusters). If it belongs to an inside cluster, we let $f(x)=1$ and declare that it belongs to $\mathcal{R}$. If it does not belong to any inside clusters, and it belongs to a boundary cluster, it uses a local function, which we describe below, to determine its location status. Finally, if it is not within any inside or boundary clusters, we let $f(x)=-1$, and declare that the $\mathrm{SU}$ is in $\mathcal{R}^{c}$.

Let $\mathcal{B}=\left\{C_{1}, \ldots, C_{M}\right\}$ be the set of boundary clusters. The boundary clusters collaboratively estimate the boundary of $\mathcal{R}$ based on local information and message exchanges between cluster heads. We use the reproducing kernel Hilbert space (RKHS) $[25,118]$ formulation to obtain a function that distinguishes a location $x$ to be inside or outside $\mathcal{R}$. However, since we do not assume that there is a central authority to perform the estimation, we consider instead finding a collection $\left\{f_{C_{j}}\right\}$ 
of local functions, each corresponds to a boundary cluster. If $x$ is not within an inside cluster and it belongs to a boundary cluster, we let the estimation function $f(x)$ take the value $f_{C}(x)$ where $C$ is chosen randomly from the set of boundary clusters containing $x$.

Let $H_{K}$ be a RKHS corresponding to a kernel $K(\cdot, \cdot)$ that serves as a similarity measure between two SU locations. We restrict to kernels that are radial basis functions (RBF), i.e., those kernels that can be expressed as functions of the Euclidean distance between two SUs. For each $C_{j} \in \mathcal{B}$, let $\mathcal{N}\left(C_{j}\right)$ be the set of indices $k$ with $j \neq k$ and $\left|C_{k} \cap C_{j}\right| \neq 0$. We call those clusters in $\mathcal{N}\left(C_{j}\right)$ the neighboring clusters of $C_{j}$. Our goal is to

$$
\begin{aligned}
\min & \sum_{i \in \cup_{j=1}^{M} C_{j}}\left(z_{i}-u_{i}\right)^{2}+\sum_{m=1}^{M} \nu_{m}\left\|f_{C_{m}}\right\|_{H_{K}}^{2} \\
& +\sum_{m=1}^{M} \sum_{k \in \mathcal{N}\left(C_{m}\right)} \eta_{m} \epsilon_{m, k}^{2}
\end{aligned}
$$

subject to

$$
\begin{aligned}
& f_{C_{m}} \in H_{K}, \forall C_{m} \in \mathcal{B}, \\
& z_{i}=f_{C_{m}}\left(x_{i}\right), \forall i \in C_{m}, C_{m} \in \mathcal{B}, \\
& \epsilon_{m, k}=\frac{1}{\left|C_{m}\right|} \sum_{i \in C_{m}} f_{C_{m}}\left(x_{i}\right)-\frac{1}{\left|C_{k}\right|} \sum_{i \in C_{k}} f_{C_{k}}\left(x_{i}\right), \\
& \forall k \in \mathcal{N}\left(C_{j}\right), C_{j} \in \mathcal{B},
\end{aligned}
$$

where $\|\cdot\|_{H_{K}}$ is the norm of $H_{K}$, and $\nu_{m}, \eta_{m}$, for $j=1, \ldots, M$, are positive constants. The minimization in (3.3) is over all variables $z_{i}, f_{C_{m}}$ and $\epsilon_{m, k}$. The constraints (3.4) 
require that the local classifier $f_{C_{m}}$ from each boundary cluster $C_{m}$ is chosen from the RKHS $H_{K}$. The constraints (3.5) ensure that if a SU belongs to multiple boundary clusters, the classification result remains the same regardless of the local classifier used. Finally, the constraints (3.6) ensure that the estimated boundary is smooth (cf. Assumption 3.1(b)).

The kernel least squares minimization problem (3.3) is similar to that proposed in [25], which considers a general distributed learning framework, but without additional constraints like (3.6). The reference [25] also provides a distributed method to iteratively obtain the optimizers $\left\{f_{C_{m}}\right\}$ by message exchanges between cluster heads. In the following, we show that their distributed algorithm can be adapted to our minimization problem (3.3). Our argument is similar to that in [25], and treats the minimization in (3.3) as projections onto closed convex subspaces of a Hilbert space. This can be done because of the successive orthogonal projection (SOP) theorem [119], which we state below without proof.

Theorem 3.1. Let $\left\{\Lambda_{m}\right\}_{m=1}^{M}$ be a set of closed, convex, and affine subsets of a RKHS $H$, and whose intersection $\Lambda=\bigcap_{m=1}^{M} \Lambda_{m}$ is nonempty. For any $v_{0} \in H$, let $v^{*}$ be the orthogonal projection of $v_{0}$ onto $\Lambda$, and for all $n \geq 1$, let $v_{n}$ be the orthogonal projection of $v_{n-1}$ onto $\Lambda_{(n \bmod M)}$. Then, $\lim _{n \rightarrow \infty}\left\|v_{n}-v^{*}\right\|=0$.

Suppose that $S=\sum_{m=1}^{M}\left|\mathcal{N}\left(C_{m}\right)\right|$ is the total number of variables $\epsilon_{m, k}$ where $m=1, \ldots, M$ and $k \in \mathcal{N}\left(C_{m}\right)$. Let $H=\mathbb{R}^{N} \times H_{K}^{M} \times \mathbb{R}^{S}$. In the sequel, to avoid cluttered notations, we let $v=\left(\left(z_{i}\right),\left(f_{m}\right),\left(\epsilon_{m, k}\right)\right) \in H$ to denote an element from $H$ with the understanding that the index $i$ runs from 1 to $N$, the index $m$ runs from 1 
to $M$, and the index $k \in \mathcal{N}\left(C_{m}\right)$ for each $m$. We let $H$ be a Hilbert space by letting the squared norm of $v$ be

$$
\|v\|_{H}^{2}=\sum_{i=1}^{N}\left|z_{i}\right|^{2}+\sum_{m=1}^{M} \nu_{m}\left\|f_{C_{m}}\right\|_{H_{K}}^{2}+\sum_{m=1}^{M} \sum_{k \in \mathcal{N}\left(C_{m}\right)} \eta_{m} \epsilon_{m, k}^{2} .
$$

For each $j=1, \ldots, M$, let

$$
\begin{gathered}
\Lambda_{j}=\left\{\left(\left(z_{i}\right),\left(f_{m}\right),\left(\epsilon_{m, k}\right)\right) \in H: z_{i}=f_{C_{m}}\left(x_{i}\right), \forall i \in C_{m},\right. \\
\quad \text { and } \\
\epsilon_{m, k}=\frac{1}{\left|C_{m}\right|} \sum_{i \in C_{m}} f_{C_{m}}\left(x_{i}\right)-\frac{1}{\left|C_{k}\right|} \sum_{i \in C_{k}} f_{C_{k}}\left(x_{i}\right), \\
\left.\quad \forall k \in \mathcal{N}\left(C_{m}\right)\right\} .
\end{gathered}
$$

It can be shown that $\Lambda_{j}$ is a closed subspace of $H$. Then, the minimization problem (3.3) is equivalent to finding the projection of $\left(u_{1}, \ldots, u_{N}, 0, \ldots, 0\right)$ onto the closed and convex set $\Lambda=\cap_{j=1}^{M} \Lambda_{j}$.

As pointed out in [25], instead of directly finding the projection onto $\Lambda$, Theorem 3.1 allows us to iteratively project onto each $\Lambda_{m}$, for $m=1, \ldots, M$. The SOP algorithm first finds the projection $v_{1}$ of $\left(u_{1}, \ldots, u_{N}, 0, \ldots, 0\right)$ onto $\Lambda_{1}$, then finds the projection of $v_{1}$ onto $\Lambda_{2}$, and so on. Projections are performed over all $\Lambda_{m}$, $m=1, \ldots, M$, with multiple iterations over the indices $m$. Suppose that at an 
iteration, we seek to project $v=\left(\left(\tilde{z}_{i}\right),\left(\tilde{f}_{j}\right),\left(\tilde{\epsilon}_{j, k}\right)\right)$ onto $\Lambda_{m}$. This is equivalent to

$$
\begin{aligned}
\min & \sum_{i \in C_{m}}\left(f_{C_{m}}\left(x_{i}\right)-\tilde{z}_{i}\right)^{2}+\nu_{m}\left\|f_{C_{m}}-\tilde{f}_{C_{m}}\right\|_{H_{K}}^{2} \\
& +\eta_{m} \sum_{k \in \mathcal{N}\left(C_{m}\right)}\left(\epsilon_{m, k}-\tilde{\epsilon}_{m, k}\right)^{2}
\end{aligned}
$$

subject to

$$
\begin{aligned}
& f_{C_{m}} \in H_{K}, \\
& \epsilon_{m, k}=\frac{1}{\left|C_{m}\right|} \sum_{i \in C_{m}} f_{C_{m}}\left(x_{i}\right)-\frac{1}{\left|C_{k}\right|} \sum_{i \in C_{k}} f_{C_{k}}\left(x_{i}\right), \\
& \forall k \in \mathcal{N}\left(C_{m}\right) .
\end{aligned}
$$

The minimization in (3.7) is over $f_{C_{m}}$ and $\epsilon_{m, k}$, and involves only data from $C_{m}$ and its neighboring clusters. It is thus a local optimization problem. Suppose that $\left(f_{C_{m}}^{*},\left(\epsilon_{m, k}^{*}\right)_{k \in \mathcal{N}\left(C_{m}\right)}\right)$ is the optimizer for $(3.7)$. The projected point is then given by $v^{*}=\left(\left(z_{i}^{*}\right),\left(f_{j}^{*}\right),\left(\epsilon_{j, k}^{*}\right)\right)$, where

$$
\begin{aligned}
& z_{i}^{*}=\tilde{z}_{i} \text { if } i \notin C_{m}, \text { and } z_{i}^{*}=f_{C_{m}}^{*}\left(x_{i}\right) \text { if } i \in C_{m}, \\
& f_{C_{j}}^{*}=\tilde{f}_{C_{j}} \text { and } \epsilon_{j, k}^{*}=\tilde{\epsilon}_{j, k} \text { if } j \neq m .
\end{aligned}
$$

The messages that cluster $C_{m}$ passes to a neighboring cluster $C_{k}$ are $\left\{z_{i}^{*}: i \in C_{m} \cap C_{k}\right\}$ and $\frac{1}{\left|C_{m}\right|} \sum_{i \in C_{m}} f_{C_{m}}\left(x_{i}\right)$, where the first message represents its current best estimate of $\left\{u_{i}: i \in C_{m} \cap C_{k}\right\}$ subject to the constraints (3.4)-(3.6), and serves as the "training labels" [25] for the SUs in both clusters. The second message encodes the average value achieved by $f_{C_{m}}^{*}$, and allows $C_{k}$ to adjust its own classifier to improve the 
smoothness of the estimated boundary. The following result is a direct consequence of the Representer Theorem [120], and its proof is omitted. It characterizes the form of the optimal solution $f_{C_{m}}^{*}$ for (3.7).

Proposition 3.1. For each $C_{m} \in \mathcal{B}$, the optimal solution to the minimization problem (3.7) is given by

$$
f_{C_{m}}^{*}(x)=\sum_{i \in C_{m}} \beta_{m, i} K\left(x, x_{i}\right) .
$$

Furthermore, if the kernel $K(\cdot, \cdot)$ is a radial basis function, the computation of $f_{C_{m}}(x)$ requires only the knowledge of $\left\|x-x_{i}\right\|$, for all $i \in C_{m}$.

From (3.7) and Proposition 3.1, to train the classifier for a cluster $C_{m} \in \mathcal{B}$, we require the cluster head to know $\left\|x_{i}-x_{j}\right\|$, for all $i, j \in C_{m}$. This can be obtained using various ranging techniques. Examples include methods in which each $\mathrm{SU} i$ broadcasts a pilot signal with known transmit power, or exchange messages with timestamps [121]. Our distributed boundary estimation algorithm is formally stated in Algorithm 3.1, which we call the DBE algorithm. The following proposition shows that the classifiers in the DBE algorithm converges.

Proposition 3.2. For each $C_{m}$, where $m=1, \ldots, M$, the sequence $\left(f_{C_{m}}^{t}\right)$ in line 9 of the DBE algorithm converges as the number of iterations $t \rightarrow \infty$.

Proof. Since each $\Lambda_{m}$ is a closed subspace of $H$, and their intersection $\Lambda=\cap_{m} \Lambda_{m}$ is nonempty, the result follows from Theorem 3.1.

The DBE algorithm presented in Algorithm 3.1 assumes that the boundary cluster 
heads are synchronized so that local projections can be performed sequentially. We note however that it is still possible to achieve convergence if after a boundary cluster head has performed its local projection, it randomly chooses a neighboring boundary cluster head to pass information to. The chosen neighboring cluster head then repeats the same procedure. We call this the randomized DBE algorithm. Let $\mathcal{G}$ be the graph with vertex set $\mathcal{B}$, which has an edge between $C_{i}$ and $C_{j}$ if they are neighboring clusters. We have the following convergence result.

Proposition 3.3. Suppose that $K(u, u) \leq \kappa^{2}$ for all $u \in H_{K}$, and $\mathcal{G}$ is connected. The estimation error in the randomized DBE algorithm converges to $\mathbb{E}\left[\left(f^{*}(X)-U\right)^{2}\right]$ where $f^{*}$ is an optimal solution to (3.3).

Proof. Let $f_{n}$ be the estimation function at the $n$-th projection of the randomized DBE algorithm. Since $\mathcal{G}$ is connected, the random sequence of chosen cluster heads is an irreducible and recurrent Markov chain so that every cluster head appears infinitely often in the random sequence. From [122], the sequence $f_{n}$ is weakly convergent to an optimal estimation function $f^{*}$. Since weakly convergent sequences are bounded [123], we have $\left|f_{n}(x)\right| \leq\left\|f_{n}\right\|_{H_{K}} \sqrt{K(x, x)} \leq \kappa\left\|f_{n}\right\|_{H_{K}}$ is bounded. From the dominated convergence theorem [115] and the reproducing property of $H_{K}$, we obtain

$$
\begin{aligned}
\lim _{n \rightarrow \infty} \mathbb{E}\left[\left(f_{n}(X)-U\right)^{2}\right] & =\mathbb{E}\left[\lim _{n \rightarrow \infty}\left(\left\langle f_{n}, K(\cdot, X)\right\rangle_{H_{K}}-U\right)^{2}\right] \\
& =\mathbb{E}\left[\left(f^{*}(X)-U\right)^{2}\right],
\end{aligned}
$$

where $\langle\cdot, \cdot\rangle_{H_{K}}$ is the inner product of $H_{K}$, and the proof is now complete. 


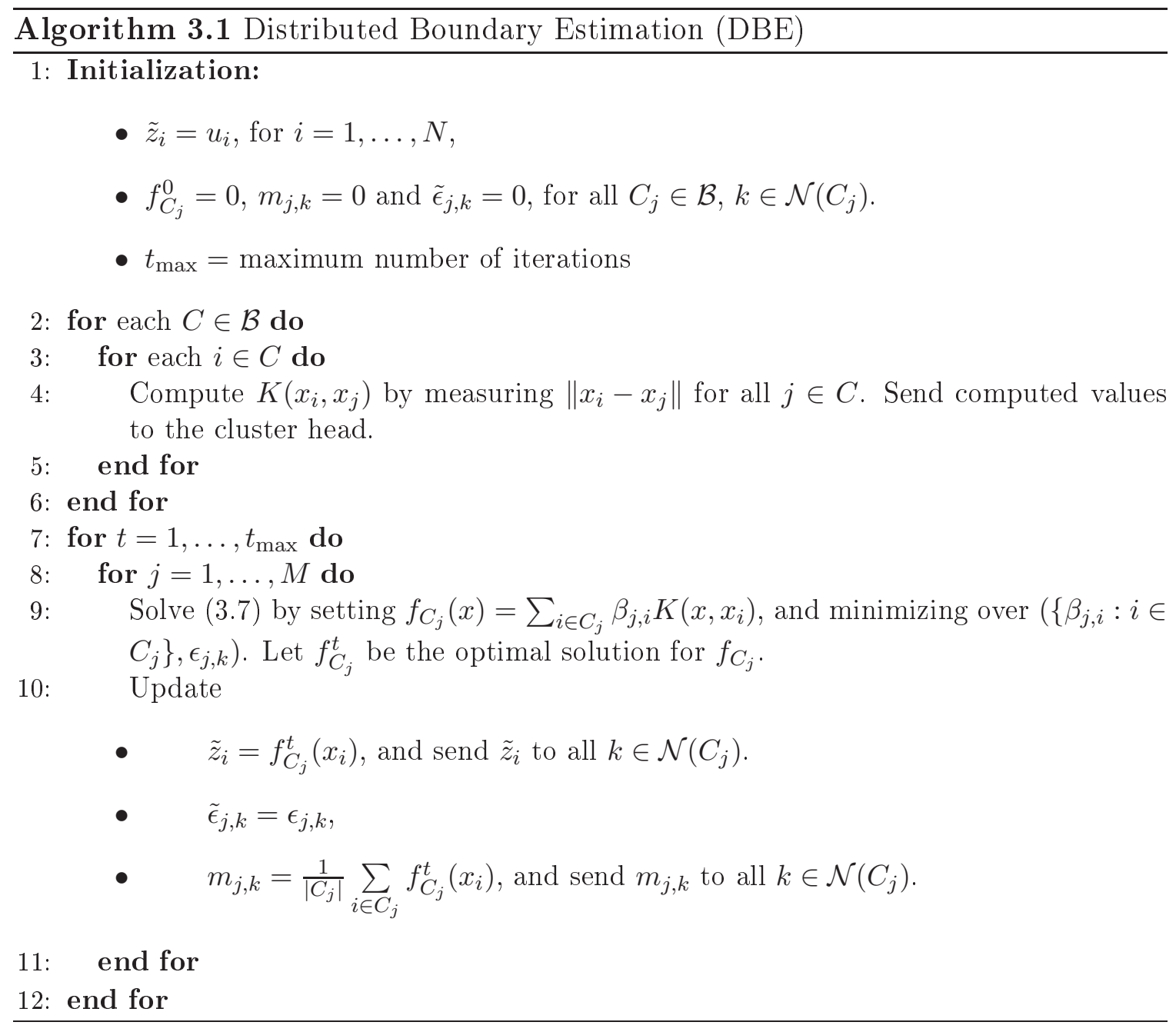

\subsection{Performance Analysis}

In this section, we first analyze the trade-off between communication cost and estimation error in the DBE algorithm. Then, we propose a two-step approach to spatial spectrum sensing based on the DBE algorithm, and compare its setup complexity and throughput with that of the traditional fusion center (FC) approach. 


\subsubsection{Communication Cost and Estimation Error}

We let the SU locations be distributed as a homogeneous Poisson point process $\Pi$ in $\mathbb{R}^{d}$ with rate $\lambda$, and assume that the region of interest $A$ has unit $d$-dimensional volume. Since we do not have any prior knowledge of the SU locations, it is reasonable to assume that SUs are located independently and randomly. The homogeneous Poisson point process captures this assumption and has been widely adopted in the literature to model the distribution of ad hoc communicating devices $[124,125]$. The Poisson point process also makes the mathematical analysis tractable, which provides insights into the system performance in practical scenarios. In Section 3.4.3, we present simulation results for a specific case when SUs are not distributed according to a homogeneous Poisson point process.

We consider the trade-off between communication cost and the estimation error resulting from the boundary estimation as the rate $\lambda$ varies, and we determine an approximate optimal density for the SUs that minimizes a weighted sum of the communication cost and estimation error. Finding the optimal density is useful in the case where there are multiple PUs, and random subsets of SUs may be chosen to estimate the boundary of each PU. Intuitively, as SUs become more dense, the expected communication cost increases because the number of SUs in each cluster and the number of boundary clusters increase, but the expected estimation error decreases due to the availability of more training examples. In the following, because of technical difficulties, we present heuristic approximations to both the expected communication cost and estimation error, and determine the optimal density by minimizing a weighted 
sum of these approximations. We present simulation results in Section 3.4 to verify that the approximate optimal density found is close to the true optimal one.

For simplicity, we assume that the boundary cluster heads all come from a fixed region $\mathcal{D}$ with volume $b>0$, that this region contains the boundary of $\mathcal{R}$, and that it is sufficiently small so that certain approximations, which we describe below, hold. In finding the optimal density, we will see that the region $\mathcal{D}$ needs not be known in advance. We summarize some of the notations introduced in this section in Table 3.2 for ease of reference.

Table 3.2: Summary of notations used for performance analysis in Chapter 3

\begin{tabular}{|c|l|}
\hline Symbol & Definition \\
\hline$\lambda$ & rate of SU location Poisson point process \\
\hline $\mathcal{D}$ & approximate region in $A$ containing all boundary clusters \\
\hline$b$ & volume of the region $\mathcal{D}$ \\
\hline$B_{x}(\delta)$ & disk of radius $\delta$ centered at $x$ \\
\hline$v_{d}$ & volume of $B_{0}(1)$ in $\mathbb{R}^{d}$ \\
\hline$g(r)$ & communication cost function between two SUs distance $r$ apart \\
\hline$p_{B}$ & $\begin{array}{l}\text { approximate probability a cluster centered in } \mathcal{D} \text { is } \\
\text { a boundary cluster }(\text { see }(3.10))\end{array}$ \\
\hline$\kappa$ & $K(u, u) \leq \kappa^{2}$ for all $u \in H_{K}$ \\
\hline
\end{tabular}

\section{Communication Cost}

Suppose that the cost of sending a message from a SU at position $x$ to another at position $x^{\prime}$ is given by a non-negative function $g\left(\left\|x-x^{\prime}\right\|\right)$ with $g(0)=0$. In many wireless applications, this cost is modeled by the power required to achieve a given signal to noise ratio at the receiver, and $g(r)$ is a function of the form $c r^{\zeta}$, where $c>0$ and $\zeta \in[2,5]$. Let a disk of radius $\delta$ centered at $x$ be denoted as $B_{x}(\delta)$, and 
let $v_{d}$ be the volume of a unit disk in $\mathbb{R}^{d}$. The expected communication cost can be found by considering the intra-cluster communication cost and the inter-cluster communication cost separately. The intra-cluster cost is incurred when SUs within a cluster communicate with their cluster head. Let the cluster head of cluster $C_{j}$ be $\bar{x}_{j}$. The intra-cluster cost is then given by

$$
\begin{aligned}
& \mathbb{E}\left[\sum_{j=1}^{M} \sum_{i \in C_{j}} g\left(\left\|x_{i}-\bar{x}_{j}\right\|\right)\right] \\
& =\mathbb{E}[M] \mathbb{E}\left[\left|C_{1}\right|\right] \mathbb{E}\left[g(\|x\|) \mathbf{1}_{\left\{x \in B_{0}(\delta)\right\}}\right] \\
& =\lambda^{3} b p_{h} v_{d} \delta^{d} G(\delta),
\end{aligned}
$$

where the first equality follows from Wald's identity [115], the expected number of boundary clusters is given by $\mathbb{E}[M]=p_{h} \lambda b$, and

$$
G(\delta)=\int_{B_{0}(\delta)} g(\|x\|) \mathrm{d} x .
$$

The inter-cluster communication cost is incurred when boundary cluster heads exchange messages during the execution of the DBE algorithm. Cluster heads form a marked Poisson process with rate $p_{h} \lambda$. Let $p_{B}(x)$ be the probability that a cluster $C$ with cluster head at $x \in \mathcal{D}$, is a boundary cluster. We make the following approximations in order to compute $p_{B}(x)$ : (i) we assume that the boundary cluster test in Section 3.2.1 does not include the observation at the cluster head; (ii) we replace the number of SUs $|C|$ in one cluster by $\mathbb{E}[|C|]=v_{d} \delta^{d}$; and (iii) we assume that every $\mathrm{SU}$ in a cluster has the same probability $\bar{\alpha}=1-\alpha$ of declaring itself to be in $\mathcal{R}^{c}$ 
(this assumption is exact for those SUs in $\mathcal{R}^{c}$, and approximately true for all SUs in a boundary cluster if the cluster radius $\delta$ is sufficiently small). It can be shown that declaring a cluster $C$ to be a boundary cluster is equivalent to requiring that $|C|(1-\gamma) \leq \mathcal{U}^{-} \leq|C| \gamma$. Under the above approximations, we then have for $x \in \mathcal{D}$,

$$
p_{B}(x) \approx \sum_{(1-\gamma) v_{d} \delta^{d} \leq k \leq \gamma v_{d} \delta^{d}} \frac{\bar{\alpha}^{k} e^{\bar{\alpha}}}{k !} \triangleq p_{B} .
$$

The expected inter-cluster communication cost is then given by

$$
\begin{aligned}
& t_{\max } \mathbb{E}\left[\sum_{x, x^{\prime} \in \mathcal{D}} g\left(\left\|x-x^{\prime}\right\|\right) \mathbf{1}_{\left\{B_{x}(\delta), B_{x^{\prime}}(\delta) \in \mathcal{B},\left\|x-x^{\prime}\right\| \leq 2 \delta\right\}}\right] \\
& \leq t_{\max } \mathbb{E}\left[\sum_{x, x^{\prime} \in \mathcal{D}} g\left(\left\|x-x^{\prime}\right\|\right) \mathbf{1}_{\left\{\left\|x-x^{\prime}\right\| \leq 2 \delta\right\}} p_{B}\right] \\
& =p_{B} p_{h}^{2} \lambda^{2} t_{\max } \int_{\mathcal{D}} \int_{B_{x}(2 \delta)} g\left(\left\|x-x^{\prime}\right\|\right) \mathrm{d} x^{\prime} \mathrm{d} x \\
& =\lambda^{2} b p_{h} p_{B} t_{\max } G(2 \delta),
\end{aligned}
$$

where the penultimate equality follows from two applications of the Slivnyak-Mecke Theorem [126]. (3.11) can capture the dependence on inter-cluster distance by the non-negative function $g\left(\left\|x-x^{\prime}\right\|\right)$, which calculates the cost of sending a message from a cluster head at position $x$ to another cluster head at position $x^{\prime}$.

From (3.9) and (3.12), the total expected communication cost per SU in $\mathcal{D}$ is then upper bounded by

$$
C(\lambda)=\lambda^{2} p_{h} v_{d} \delta^{d} G(\delta)+\lambda p_{h} p_{B} t_{\max } G(2 \delta) .
$$


And the communication errors or losses can be counted inside the communication functions $g\left(\left\|x_{i}-\bar{x}_{j}\right\|\right)$ in (3.8) and $g\left(\left\|x-x^{\prime}\right\|\right)$ in (3.11), which describe the amount of average cost to obtain successful communication between two SUs.

\section{Estimation Error}

To evaluate the estimation error $\mathcal{E}$ in (3.2), we consider

$$
\mathbb{E}\left[\left(f^{*}(X)-U\right)^{2} \mathbf{1}_{\{X \in \mathcal{D}\}}\right]=b \mathcal{E}_{\mathcal{D}}
$$

where

$$
\mathcal{E}_{\mathcal{D}}=\mathbb{E}\left[\left(f^{*}(X)-U\right)^{2} \mid X \in \mathcal{D}\right],
$$

and $f^{*}$ is the solution to (3.3) given the data $y=\left\{\left(x_{i}, u_{i}\right): i=1, \ldots, N\right\}$. Compared to $\mathcal{E}$ in (3.2), we have ignored the estimation errors incurred in clusters close to the PU or far away from the no-talk region boundary. This is because for sufficiently

large rate $\lambda$, these errors are largely dependent on the detection threshold instead of the rate.

Unfortunately, to the best of our knowledge, finding high probability bounds for the generalization error of learning problems like (3.3) is an open problem, because of correlations in the loss functions for the clusters due to constraints (3.5) and (3.6). We therefore make a simplification by dropping these constraints in our analysis, and assume the boundary clusters perform their learning independently of each other. 
Furthermore, for a boundary cluster $C_{j}$, let $f_{C_{j}}^{*}$ be the local estimation function corresponding to $f^{*}$, and we approximate (3.15) using

$$
\begin{aligned}
\tilde{\mathcal{E}}_{\mathcal{D}} & =\mathbb{E}\left[\frac{1}{M} \sum_{j=1}^{M}\left(f_{C_{j}}^{*}(X)-U\right)^{2} \mathbf{1}_{\left\{X \in C_{j}\right\}} \mid X \in \mathcal{D}\right] \\
& =\frac{v_{d} \delta^{d}}{b M} \sum_{j=1}^{M} R_{j}
\end{aligned}
$$

where $R_{j}=\mathbb{E}\left[\left(f_{C_{j}}^{*}(X)-U\right)^{2} \mid X \in C_{j}\right]$.

We assume that the kernel $K$ satisfies the bound $K(u, u) \leq \kappa^{2}$ for all $u \in H_{K}$, and for some constant $\kappa>0$. We also assume that $\nu_{j}=\nu\left|C_{j}\right|$ for all $j=1, \ldots, M$, and some positive constant $\nu$. We first state two lemmas, the first of which follows from the Chernoff bound, and the second from Lemma 23, Theorems 12 and 22 of [127]. We omit their proofs here.

Lemma 3.1. For any measurable set $C$, let $N(C)$ and $\mu(C)$ be the count function and mean measure of the Poisson point process $\Pi$, respectively. For any $\varepsilon>0$, we have

$$
\mathbb{P}(|N(C)-\mu(C)| \geq \varepsilon) \leq 2 e^{-\frac{1}{4} \varepsilon^{2} \mu(C)} .
$$

Lemma 3.2. Suppose that $K(u, u) \leq \kappa^{2}$ for all $u \in H_{K}$. Then, for any $j=1, \ldots, M$, and any $\varepsilon>0$, with probability at least $1-\varepsilon$ over the random draw of the data $y$, 
we have

$$
\begin{aligned}
R_{j} & \leq \frac{1}{\left|C_{j}\right|} \sum_{i \in C_{j}}\left(f_{C_{j}}^{*}\left(x_{i}\right)-u_{i}\right)^{2}+\frac{4 \kappa^{2}}{\nu\left|C_{j}\right|}\left(\frac{\kappa}{\sqrt{\nu}}+1\right)^{2} \\
& +\left(\frac{8 \kappa^{2}}{\nu}+1\right)\left(\frac{\kappa}{\sqrt{\nu}}+1\right)^{2} \sqrt{\frac{\ln (1 / \varepsilon)}{2\left|C_{j}\right|}}
\end{aligned}
$$

For simplicity, we approximate $M \approx p_{h} \lambda b$. From Lemma 3.1, if $\lambda$ is sufficiently large, we have for any region $C,(1-\varepsilon) \mu(C) \leq N(C) \leq(1+\varepsilon) \mu(C)$ with high probability. Therefore, by choosing $\lambda$ to be large enough, with probability at least $1-\varepsilon$, where $\varepsilon \in(0,1)$, we have for all $j=1, \ldots, M$,

$$
\begin{aligned}
R_{j} & \leq \frac{1}{\lambda(1-\varepsilon) v_{d} \delta^{d}} \sum_{i \in C_{j}}\left(f_{C_{j}}^{*}\left(x_{i}\right)-u_{i}\right)^{2} \\
& +\frac{4 \kappa^{2}}{\nu \lambda(1-\varepsilon) v_{d} \delta^{d}}\left(\frac{\kappa}{\sqrt{\nu}}+1\right)^{2} \\
& +\left(\frac{8 \kappa^{2}}{\nu}+1\right)\left(\frac{\kappa}{\sqrt{\nu}}+1\right)^{2} \sqrt{\frac{\ln \left(p_{h} \lambda b / \varepsilon\right)}{2 \lambda(1-\varepsilon) v_{d} \delta^{d}}} .
\end{aligned}
$$

Using the probability union bound, we have with probability at least $1-\varepsilon$,

$$
\begin{aligned}
\tilde{\mathcal{E}}_{\mathcal{D}} & \leq \frac{1}{\lambda(1-\varepsilon) b M} \sum_{i}\left(f^{*}\left(x_{i}\right)-u_{i}\right)^{2}+\frac{4 \kappa^{2}}{\nu \lambda(1-\varepsilon) b}\left(\frac{\kappa}{\sqrt{\nu}}+1\right)^{2} \\
& +\frac{1}{b}\left(\frac{8 \kappa^{2}}{\nu}+1\right)\left(\frac{\kappa}{\sqrt{\nu}}+1\right)^{2} \sqrt{\frac{v_{d} \delta^{d} \ln \left(p_{h} \lambda b / \varepsilon\right)}{2 \lambda(1-\varepsilon)}} .
\end{aligned}
$$

Furthermore, Lemma 23 of [127] yields

$$
\left(f_{C_{j}}^{*}(x)-u\right)^{2} \leq\left(\frac{\kappa}{\sqrt{\nu}}+1\right)^{2}
$$


for all $j=1, \ldots, M, x \in \mathcal{D}$, and $u \in\{-1,1\}$. This implies that with probability one, $\tilde{\mathcal{E}}_{\mathcal{D}}$ is upper bounded by the right hand side of (3.16) plus $\varepsilon(\kappa / \sqrt{\nu}+1)^{2}$.

We aim to find $\lambda>0$ that minimizes a weighted sum of the communication cost upper bound (3.13) and the estimation error upper bound given by (3.14) and (3.16). The objective function to be minimized is given by

$$
\begin{aligned}
& C(\lambda)+\beta\left(\frac{F_{3}}{\lambda}+F_{4} \sqrt{\frac{\ln \lambda}{\lambda}}\right) \\
& =F_{1} \lambda^{2}+F_{2} \lambda+\beta\left(\frac{F_{3}}{\lambda}+F_{4} \sqrt{\frac{\ln \lambda}{\lambda}}\right),
\end{aligned}
$$

where $\beta>0$ is a constant, and

$$
\begin{aligned}
& F_{1}=p_{h} v_{d} \delta^{d} G(\delta), \\
& F_{2}=p_{h} p_{B} t_{\max } G(2 \delta), \\
& F_{3}=\frac{4 \kappa^{2}}{\nu}\left(\frac{\kappa}{\sqrt{\nu}}+1\right)^{2}, \\
& F_{4}=\left(\frac{8 \kappa^{2}}{\nu}+1\right)\left(\frac{\kappa}{\sqrt{\nu}}+1\right)^{2} \sqrt{\frac{(1-\varepsilon) v_{d} \delta^{d}}{2}} .
\end{aligned}
$$

We have made the approximation that $\ln \left(p_{h} b / \varepsilon\right) / \lambda \approx 0$ when $\lambda$ is sufficiently large so that the value of $b$ needs not be known a priori. The optimal rate can be found by setting the derivative with respect to $\lambda$ of (3.17) to zero (it is clear that there is a positive minimizer) to obtain

$$
4 F_{1} \lambda^{\frac{5}{2}}+2 F_{2} \lambda^{\frac{3}{2}}+\beta\left(-2 F_{3} \lambda^{-\frac{1}{2}}+F_{4}\left((\ln \lambda)^{-\frac{1}{2}}-(\ln \lambda)^{\frac{1}{2}}\right)\right)
$$




$$
=0,
$$

the solution of which can be computed numerically. To find the optimal SU density that minimizes the communication cost subject to the constraint that the estimation error is below a given level is equivalent to (3.17), where $\beta$ is a Lagrange multiplier.

\subsubsection{Setup Complexity and Throughput}

Let $\hat{\mathcal{R}}$ be the estimator for $\mathcal{R}$ produced by the DBE algorithm. Those SUs outside of $\hat{\mathcal{R}}$ can utilize the spectrum without performing spectrum sensing, while SUs inside of $\hat{\mathcal{R}}$ perform collaborative spectrum sensing by sending their local sensing decisions to a fusion center. For convenience, we call our two-step approach the DBE-spectrum sensing (DBE-SS) method. We analyze the complexity and throughput of the DBESS method and the traditional FC method, where all SUs send their local sensing decisions to a fusion center. In the FC method, SUs do not know the boundary of $\mathcal{R}$, therefore the spectrum is utilized by the SUs only if the fusion center declares that the $\mathrm{PU}$ is inactive.

We first consider the complexity of performing boundary estimation using the DBE algorithm. Recall that each SU nominates itself to be a cluster head with probability $p_{h}$, and each cluster is covered by a disk of radius $\delta$. Therefore, there are on average $O\left(\delta^{d}\right)$ SUs in a cluster and line 4 in the DBE algorithm has complexity $O\left(\delta^{2 d}\right)$. The optimization problem $(3.7)$ can be viewed as a convex quadratic program with $O\left(\delta^{d}\right)$ constraints, with complexity $O\left(\delta^{d} \delta^{3 d}\right)=O\left(\delta^{4 d}\right)$. The expected number of 
boundary clusters is bounded by $O\left(p_{h} N\right)$, therefore the overall expected complexity of the DBE algorithm is $O\left(p_{h} N \delta^{4 d}\right)$. On the other hand, in the FC approach, SUs route their local decisions to a fusion center using a minimum spanning tree (MST). If we assume that the underlying communication network is formed by joining any two SUs that are within distance $\delta$ of each other, then the complexity of setting up a MST (with a global knowledge of the whole network topology) is $O\left(N \delta^{d}\right)$. Clearly, the DBE algorithm has higher complexity than the fusion center setup if $p_{h}>\delta^{-3 d}$.

We now compare the throughput of the DBE-SS method with that achieved by the FC approach. We make several assumptions to simplify the analysis. Suppose that all SUs transmit at the same power $P_{s}<P_{0}$, and that in any given area, at most a fraction $q$ of the SUs can share the spectrum. We assume additive white Gaussian noise channels with noise power $N_{0}$. We also assume that interference between the PU and the SUs outside of $\mathcal{R}$ is negligible, while the throughput for SUs in $\mathcal{R}$ when the $\mathrm{PU}$ is active is negligible. Then, the throughput of a $\mathrm{SU}$ in the absence of the $\mathrm{PU}$ is [128] $\bar{R}_{0}=\log _{2}\left(1+\frac{P_{s}}{N_{0}}\right)$. We further assume that each SU uses a periodic frame structure of duration $T$, which includes a sensing duration of $\tau$. Suppose that the PU is active with probability $\pi$. Then, the average throughput per user under the FC method is

$$
R_{s}^{\mathrm{FC}}=(1-\pi) q \bar{R}_{0}\left(1-\frac{\tau}{T}\right)\left(1-Q_{f}^{\mathrm{FC}}\right),
$$

where $Q_{f}^{\mathrm{FC}}$ is the false alarm rate at the fusion center. As the fusion center does not know the receiver operating characteristic (ROC) of each sensor, it uses a simple 
$k$-out-of- $N$ rule to fuse the SUs' local decisions. The false alarm is given by ${ }^{7}$

$$
Q_{f}^{\mathrm{FC}}=\sum_{i=1}^{k}\left(\begin{array}{c}
q N \\
i
\end{array}\right) \alpha^{i}(1-\alpha)^{q N-i} .
$$

The value of $k$ is chosen so that the probability of detection $Q_{d}^{\mathrm{FC}} \geq \bar{\beta}$, for some fix threshold $\bar{\beta}$.

On the other hand, the average throughput per user for the SUs under DBE-SS is

$$
\begin{aligned}
R_{s} & =(1-\pi) q \bar{R}_{0}\left(\frac{|\hat{\mathcal{R}}|}{N}\left(1-\frac{\tau}{T}\right)\left(1-Q_{f}\right)+1-\frac{|\hat{\mathcal{R}}|}{N}\right) \\
& +\pi q \bar{R}_{0}\left(1-\frac{|\mathcal{R} \cup \hat{\mathcal{R}}|}{N}\right),
\end{aligned}
$$

where $Q_{f}$ is the false alarm rate for the SUs inside $\hat{\mathcal{R}}$, with the same minimum probability of detection $\bar{\beta}$, and can be computed in a similar manner as (3.18). It can be shown that if $\hat{\mathcal{R}} \approx \mathcal{R}$ and $|\mathcal{R}| / N$ is sufficiently small, then $R_{s}>R_{s}^{\mathrm{FC}}$. This is intuitively clear as there is little benefit to perform boundary estimation if $\mathcal{R}$ covers almost all the SUs.

Next, we derive the throughput for the PU. In the FC approach, the PU average throughput is

$$
\begin{aligned}
R_{p}^{\mathrm{FC}} & =\pi\left(Q_{d}^{\mathrm{FC}} \log _{2}\left(1+\frac{P_{0}}{N_{0}}\right)\right. \\
& \left.+\left(1-Q_{d}^{\mathrm{FC}}\right) \log _{2}\left(1+\frac{P_{0}}{N_{0}+q N P_{s}}\right)\right),
\end{aligned}
$$

\footnotetext{
${ }^{7}$ To avoid cluttered expressions, we assume that $q N$ is always an integer.
} 
where $Q_{d}^{\mathrm{FC}}$ is the detection probability at the fusion center. Because of estimation errors in the DBE-SS scheme, $\mathcal{R} \backslash \hat{\mathcal{R}}$ may be non-empty. Interference from SUs in this set is bounded by $N_{1}=q|\mathcal{R} \backslash \hat{\mathcal{R}}| P_{s}$. The PU average throughput under the DBE-SS scheme is then given by

$$
\begin{aligned}
R_{p} & =\pi\left(Q_{d} \log _{2}\left(1+\frac{P_{0}}{N_{0}+N_{1}}\right)\right. \\
& \left.+\left(1-Q_{d}\right) \log _{2}\left(1+\frac{P_{0}}{N_{0}+q|\mathcal{R}| P_{s}}\right)\right),
\end{aligned}
$$

where $Q_{d} \geq \bar{\beta}$ is the detection probability for the SUs inside $\hat{\mathcal{R}}$. In Section 3.4, we present simulation results to compare the throughput $R_{s}$ under the DBE-SS scheme with $R_{s}^{\mathrm{FC}}$ under the FC method, when $R_{p}=R_{p}^{\mathrm{FC}}$.

\subsection{Simulation Results}

In this section, we present simulation results to verify the performance of the DBE algorithm and the DBE-SS method. In each simulation run, 1000 sensors are uniformly distributed in a region $A$ of size $5 \times 5 \mathrm{~km}^{2}$, with the PU (e.g., a TV transmitter) located at the center of the region. We use the standard CCIR model [129] for the path loss. For each data point, we perform 1000 simulation runs using the parameters in Table 3.3. 
Table 3.3: Simulation parameters in Chapter 3

\begin{tabular}{|l|l|}
\hline Parameters & Value \\
\hline PU transmit power $P_{0}$ & $40 \mathrm{dBm}$ \\
\hline PU transmitter antenna height & $30 \mathrm{~m}$ \\
\hline PU transmit antenna gain & $6 \mathrm{dBd}$ \\
\hline PU active probability $\pi$ & 0.3 \\
\hline SU transmit power $P_{s}$ & $10 \mathrm{dBm}$ \\
\hline SU transmitter antenna height & $3 \mathrm{~m}$ \\
\hline SU transmit antenna gain & $0 \mathrm{dBd}$ \\
\hline SU coverage radius $\delta$ & $0.25 \mathrm{~km}$ \\
\hline SU false alarm probability $\alpha$ & 0.05 \\
\hline interference threshold $\theta_{0}$ & $-75 \mathrm{dBm}$ \\
\hline shadow fading standard deviation $\sigma$ & $6 \mathrm{~dB}$ \\
\hline frame duration $T$ & $20 \mathrm{~ms}$ \\
\hline sensing time $\tau$ & $1 \mathrm{~ms}$ \\
\hline learning parameters $\nu_{1}=\ldots=\nu_{M}$ & 1 \\
\hline learning parameters $\eta_{1}=\ldots=\eta_{M}$ & 10 \\
\hline learning kernel $K\left(x, x^{\prime}\right)$ & $e^{-0.5\left\|x-x^{\prime}\right\|^{2}}$ \\
\hline communication cost function $g(r)$ & $r^{2}$ \\
\hline noise power $N_{0}$ & $-10 \mathrm{dBm}$ \\
\hline estimation error parameter $\varepsilon$ & 0.01 \\
\hline trade-off weight $\beta$ & 500 \\
\hline
\end{tabular}

\subsubsection{Estimation Error and Communication Cost}

We compare the communication cost incurred and the estimation performance of the DBE algorithm with that of various benchmark algorithms, including the following:

1. Centralized boundary estimation algorithm based on LS-SVM [82]: a global classifier is trained based on information from all SUs in the boundary clusters.

2. Centralized image processing based seeded region growing (SRG) algorithm [130]: we regard the decision $u_{i}$ of each $\mathrm{SU} i$ as a pixel gray level in a binary image and segment the image by growing a region from a seed point using an 
intensity mean measure.

3. Distributed Bayesian event region detection (ERD) algorithm [83,84]: a threshold decision scheme is applied to correct the errors of local SU decisions. We refer the reader to [83] for details.

The estimation performance is evaluated according to (3.2), normalized by four times the area of $\mathcal{R}$. Since the estimation function $f$ takes values close to 1 or -1 , the normalized estimation error is approximately the area in which misclassification occurs, expressed as a fraction of the area of $\mathcal{R}$. The communication cost is computed by assuming that each message passed between two SUs a distance $r$ apart incurs a cost of $g(r)$ based on $r^{2}$.

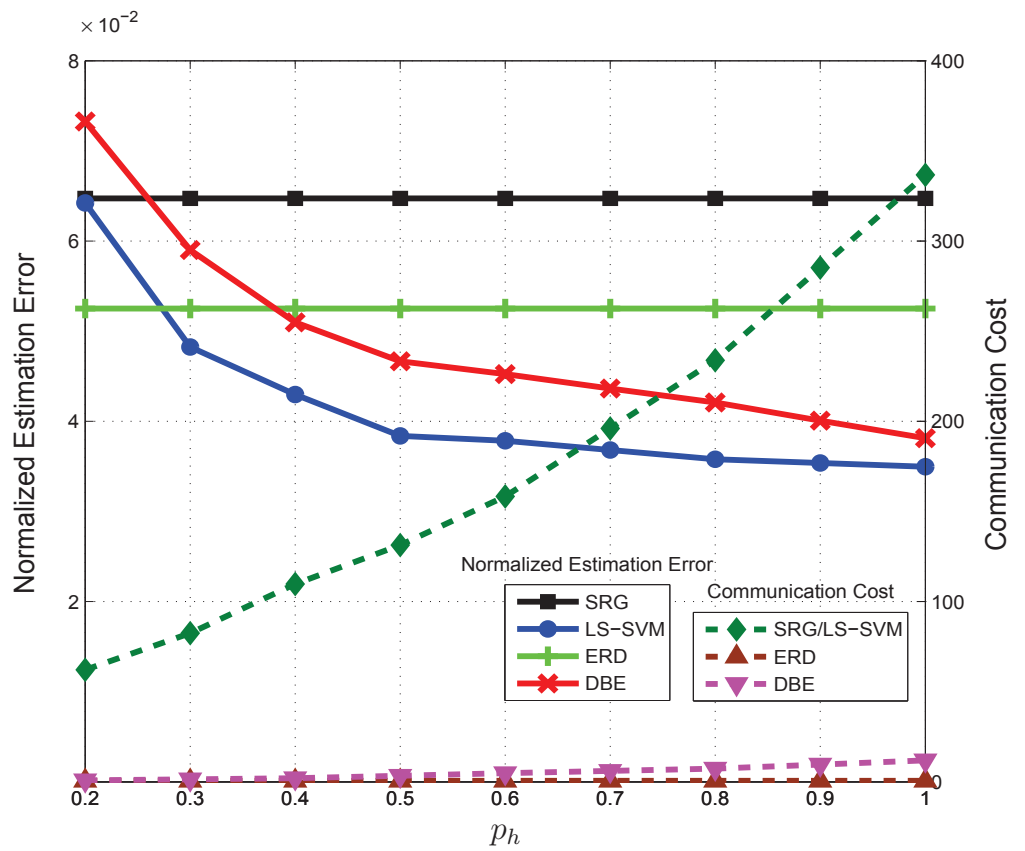

Figure 3-2: Normalized estimation errors and total communication costs for different values of $p_{h}$ when $\gamma=0.6$.

Figure 3-2 shows the normalized estimation error and communication cost for 
each algorithm when choosing different values for $p_{h}$, which is the probability that each SU independently nominates itself to be a cluster head. The threshold $\gamma$ in the boundary cluster decision rule in Section 3.2.1 is set to be 0.6. As $p_{h}$ increases, the performance of the SRG and ERD algorithms remain constant as these algorithms do not use clustering. The performance of our proposed DBE algorithm on the other hand, becomes better than the SRG and the ERD algorithms, but still underperforms the centralized LS-SVM algorithm. Centralized methods like LS-SVM and SRG however have higher communication costs than the ERD and DBE algorithms as only short range communications are required for the latter algorithms. We see that the DBE algorithm achieves arguably the best trade-off between the estimation error and communication cost if $p_{h}$ is chosen to be sufficiently large.

We now set the probability $p_{h}=0.8$ and vary the threshold $\gamma$ in the boundary cluster decision rule, with a higher value of $\gamma$ corresponding to more clusters being chosen as the boundary clusters. In Figure 3-3, we find that although the estimation error decreases with increasing $\gamma$, the rate of decrease is not very significant when $\gamma>0.6$. This is because most of the actual boundary clusters have already been included for reasonable values of $\gamma$. This implies that in practice, a reasonably value of $\gamma$ can be chosen to reduce the communication costs incurred during boundary estimation.

We next fix $p_{h}=0.8$ and $\gamma=0.6$, and vary the SU density from 5 to $160 \mathrm{SU}$ per $\mathrm{km}^{2}$. In Figure 3-4, we use simulation to compute the sum of the communication cost and estimation error (3.2) weighted by $\beta$. For comparison, we also plot the cost function $C(\lambda)$ in (3.17). Although $C(\lambda)$ does not include the estimation errors 


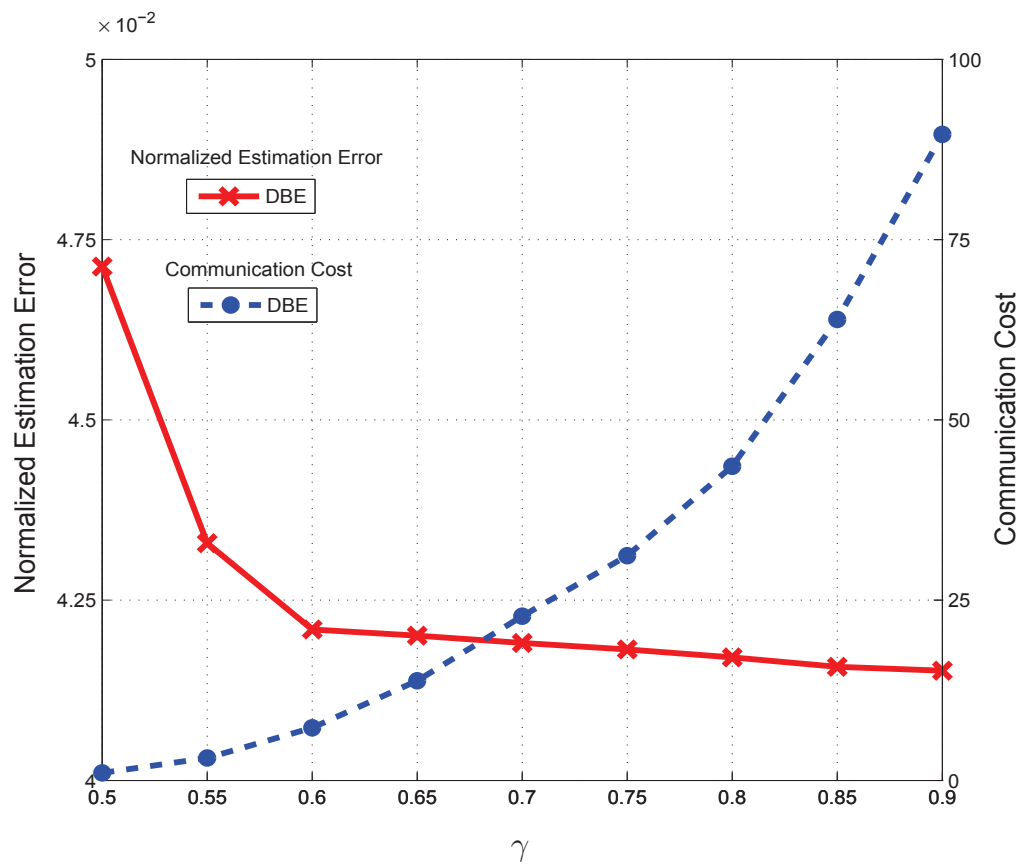

Figure 3-3: Normalized estimation errors and total communication costs when $p_{h}=$ 0.8 , and the threshold $\gamma$ is varied.

incurred in clusters far away from the boundary of $\mathcal{R}$, it is seen that it still serves as an upper bound to the simulated cost as estimation errors in clusters located far from the boundary are very small. We see from Figure 3-4 that the simulated and theoretical optimal SU densities are nearly identical to each other.

In Figure 3-5, we show the convergence of the normalized estimation error with respect to the number of inter-cluster communications for a particular simulation run with 70 boundary clusters. We see that for the DBE algorithm the estimation error converges within 200 messages (or equivalently about 3 passes over all boundary clusters). This shows that in practice, $t_{\max }$ in the DBE algorithm can be chosen to be a reasonably small value. The randomized DBE algorithm on the other hand requires a much larger number of inter-cluster communications, but less coordination amongst the boundary cluster heads. 


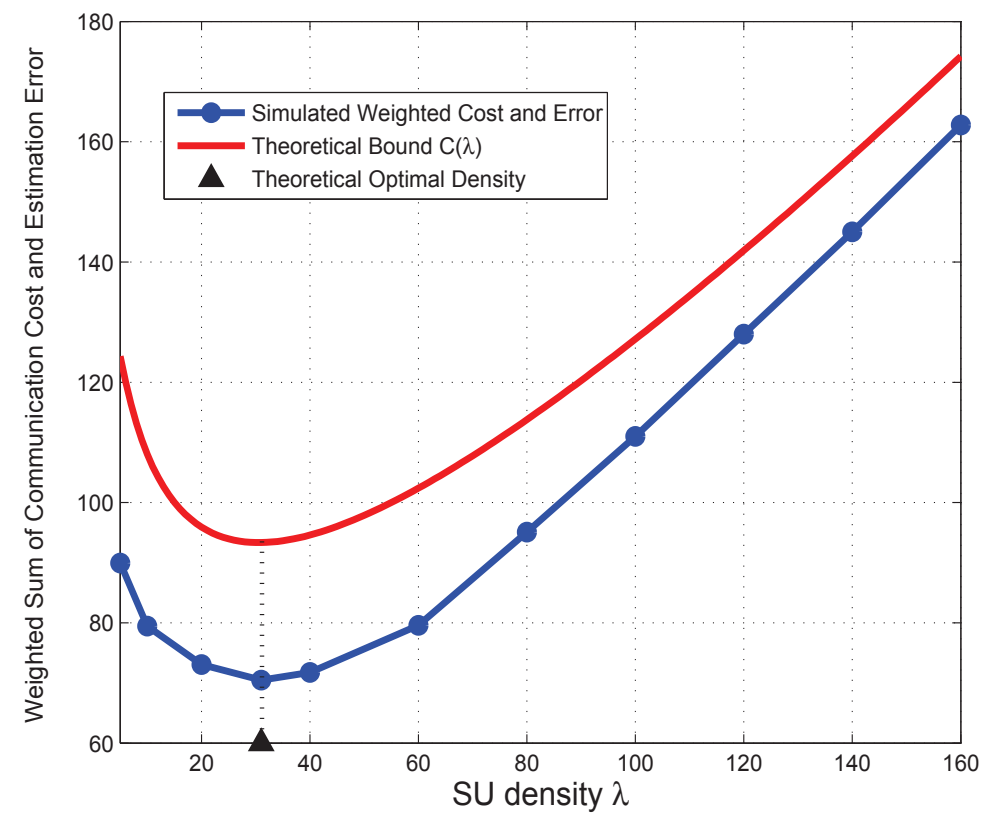

Figure 3-4: Weighted sum of communication cost and estimation error when $\gamma=0.6$ and $p_{h}=0.8$, with varying $\mathrm{SU}$ density.

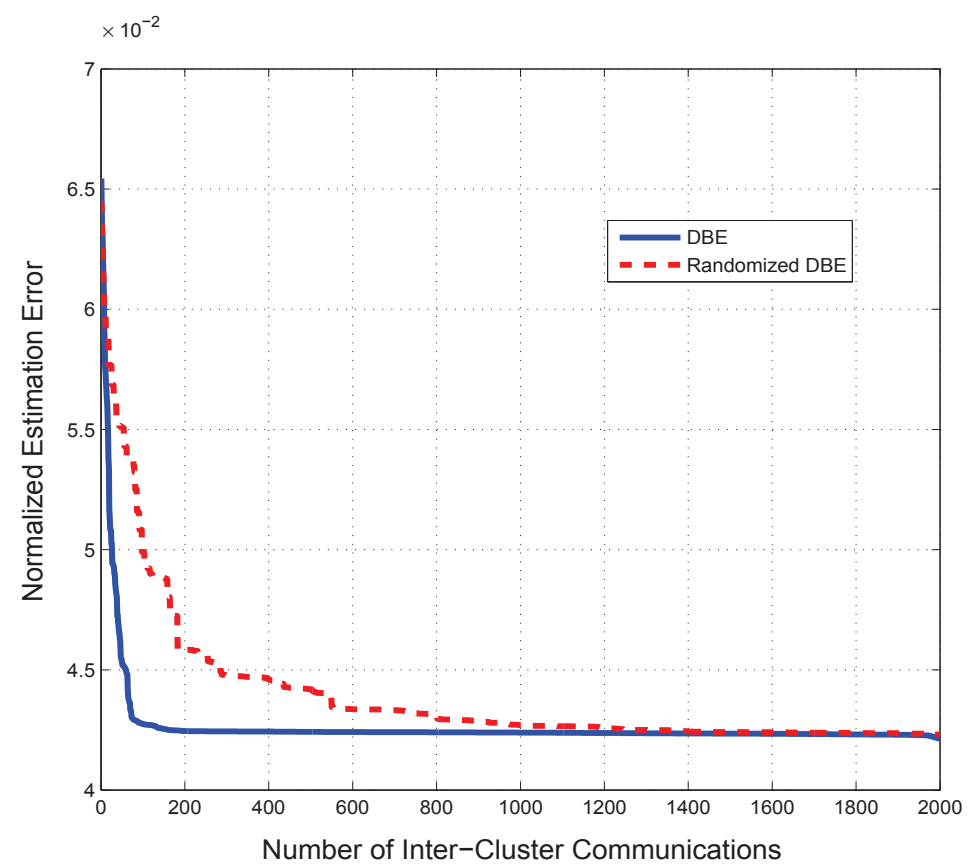

Figure 3-5: Normalized estimation error versus number of inter-cluster communications. 


\subsubsection{Throughput}

In this subsection, we present numerical results for the ROCs and throughputs of the FC and DBE-SS methods after boundary estimation with $p_{h}=0.8$ and $\gamma=0.6$. Recall that the fusion center has no knowledge of the ROC of individual SU, and a simple $k$-out-of- $N$ fusion rule is utilized in place of optimal fusion. Figure 3-6 shows the ROC curves of the two methods. It is seen that the DBE-SS method has a higher detection probability for each false alarm probability because only information from SUs in $\mathcal{R}$ are utilized, leading to less errors.

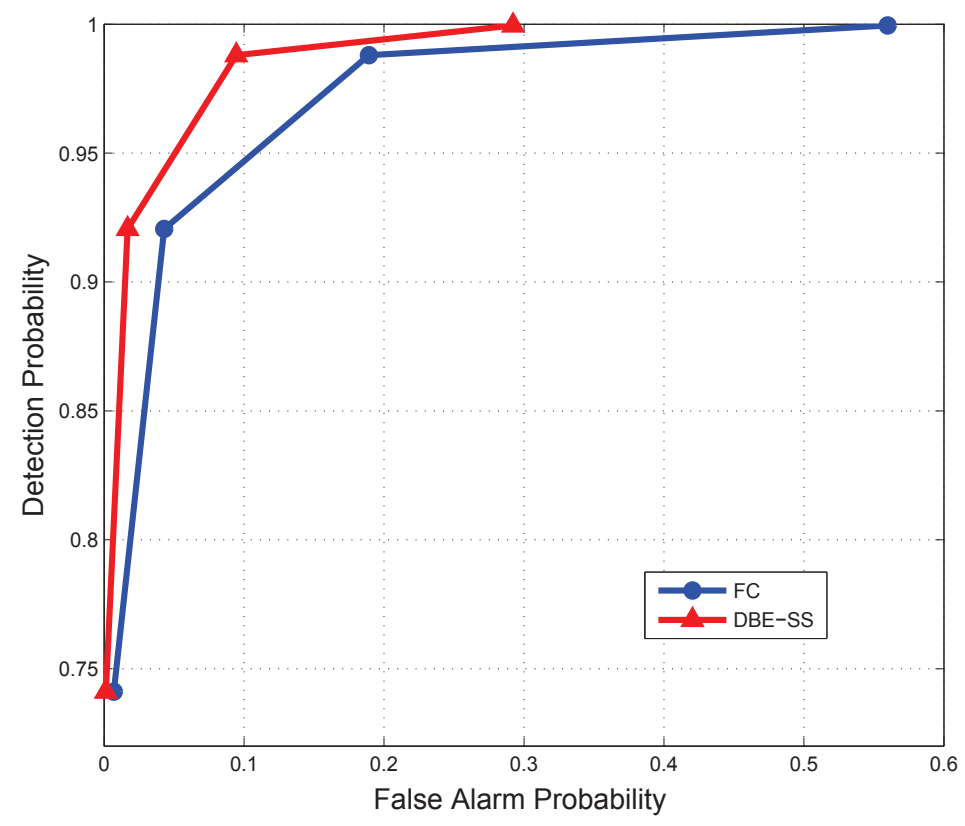

Figure 3-6: ROC curves under FC and DBE-SS.

In Figure 3-7, we let SU's false alarm probability $\alpha=0.05$ and plot the PU throughput versus the throughput per SU for both DBE-SS and FC methods. The throughput per SU for the DBE-SS method is relatively flat over all PU throughputs as SUs outside $\hat{\mathcal{R}}$ can transmit regardless of whether the PU is present or not. We 


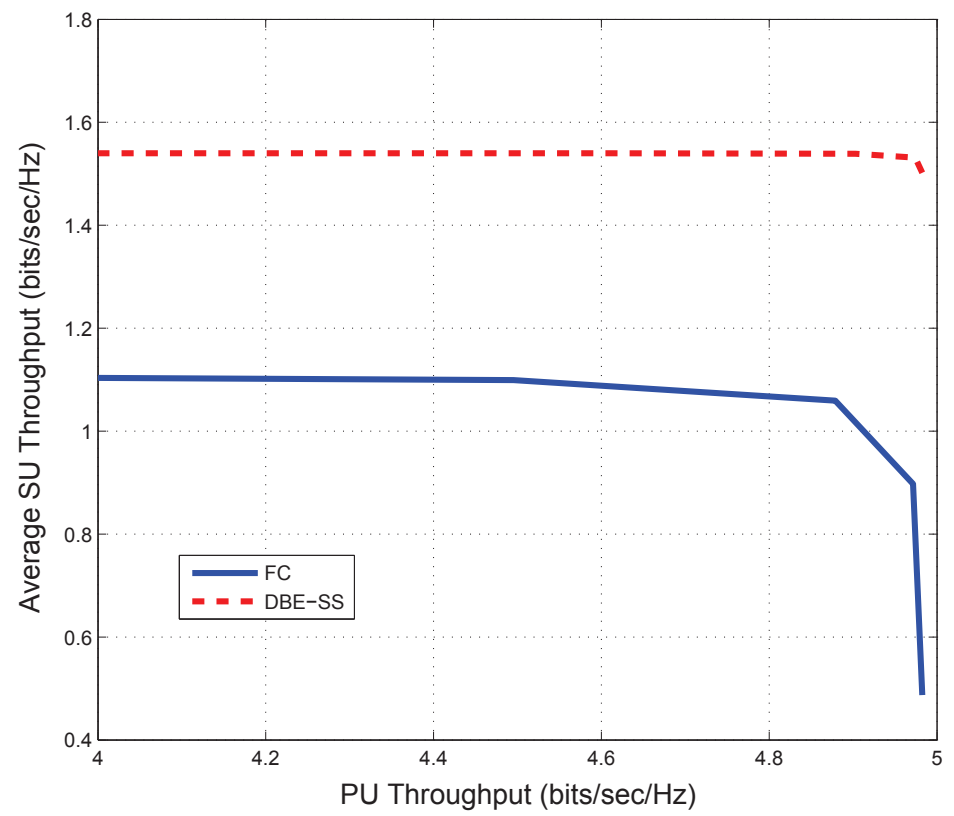

Figure 3-7: PU throughput and throughput per SU under FC and DBE-SS.

also see that the SU throughput is higher than that for the FC method. Figure 3-8 shows the average $\mathrm{SU}$ throughput when the $\mathrm{PU}$ throughput is fixed at $4 \mathrm{bits} / \mathrm{sec} / \mathrm{Hz}$, and the volume of $A$ is decreasing. We see that the DBE-SS method should only be used if $A$ is more than $10 \%$ larger than $\mathcal{R}$.

\subsubsection{Robustness}

We now compare the robustness of the various boundary estimation algorithms. We fix $p_{h}=0.8$ and $\gamma=0.6$. To simulate SU sensing errors, a boundary cluster is randomly chosen with probability $\varsigma$, and then in the chosen cluster, we flip a subset of the SU sensing decisions from -1 to 1 , while an equal number of SU sensing decisions is changed from 1 to -1 . We plot the average normalized estimation error in Figure 3-9. Figure 3-9 shows that our proposed DBE algorithm is more robust than the 


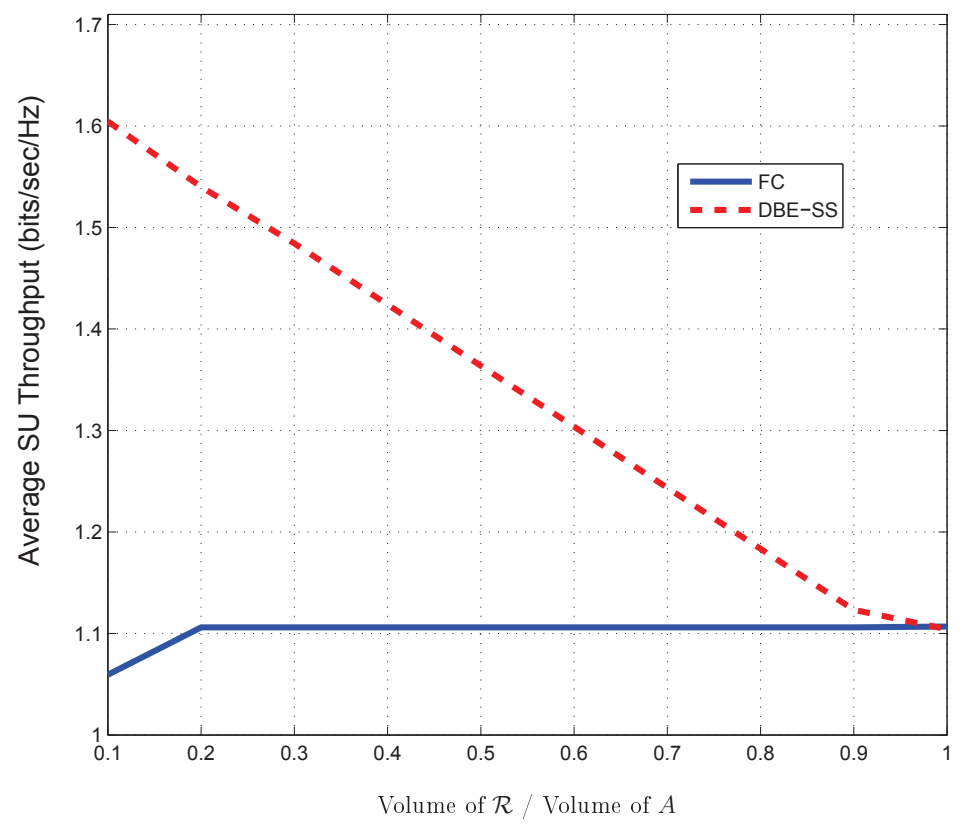

Figure 3-8: Average throughput per SU as volume of $A$ changes.

other benchmark boundary estimation methods, except for the centralized LS-SVM method. We also compare with a modified version of the DBE algorithm in which we set $\eta_{j}=0$ for all $j=1, \ldots, M$ so that the smoothness constraint (3.6) no longer applies. We see that including the smoothing constraint improves the robustness of our algorithm as neighboring boundary clusters moderate their local classifiers to avoid an abrupt change in the average classification function value within their clusters.

Next, we compare the estimation error of the DBE algorithm with and without the smoothness constraint (3.6) when the SUs are no longer distributed as a homogeneous Poisson point process. With probability $\omega$, a boundary cluster is independently populated with 20 SUs uniformly distributed inside the cluster. With probability $1-\omega$, a boundary cluster is divided into four quadrants, and a quadrant is chosen randomly. 


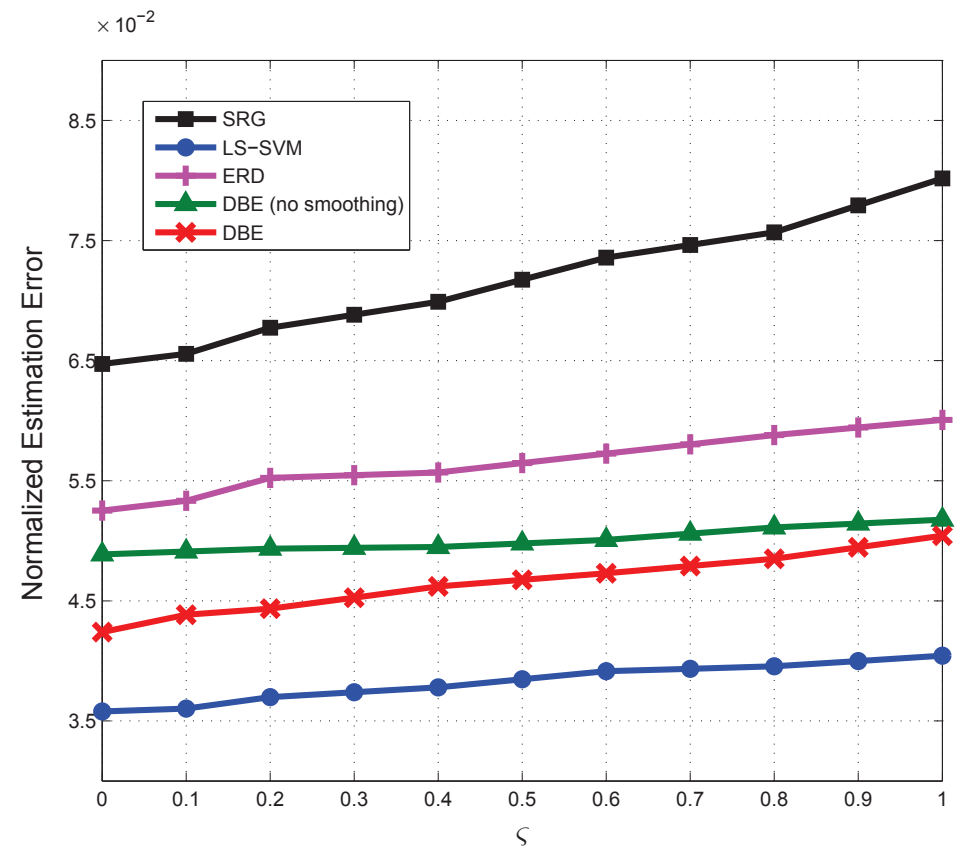

Figure 3-9: Robustness comparison of boundary estimation algorithms after flipping the observations.

The chosen quadrant is then populated with 20 SUs uniformly. In Figure 3-10, we see that the smoothing constraint results in a lower estimation error. Figure 3-11 shows a portion of the estimated boundaries.

\subsection{Summary}

In this chapter, we have developed a distributed learning method for estimating the boundary of the no-talk region of the PU to opportunistically access spectrum in a cognitive radio network, and analyzed the trade-offs between the communication cost and estimation error of our proposed learning method. We derived approximate upper bounds for the communication cost and estimation error, and provided a method to compute the optimal SU density. Simulation results suggest that our proposed 


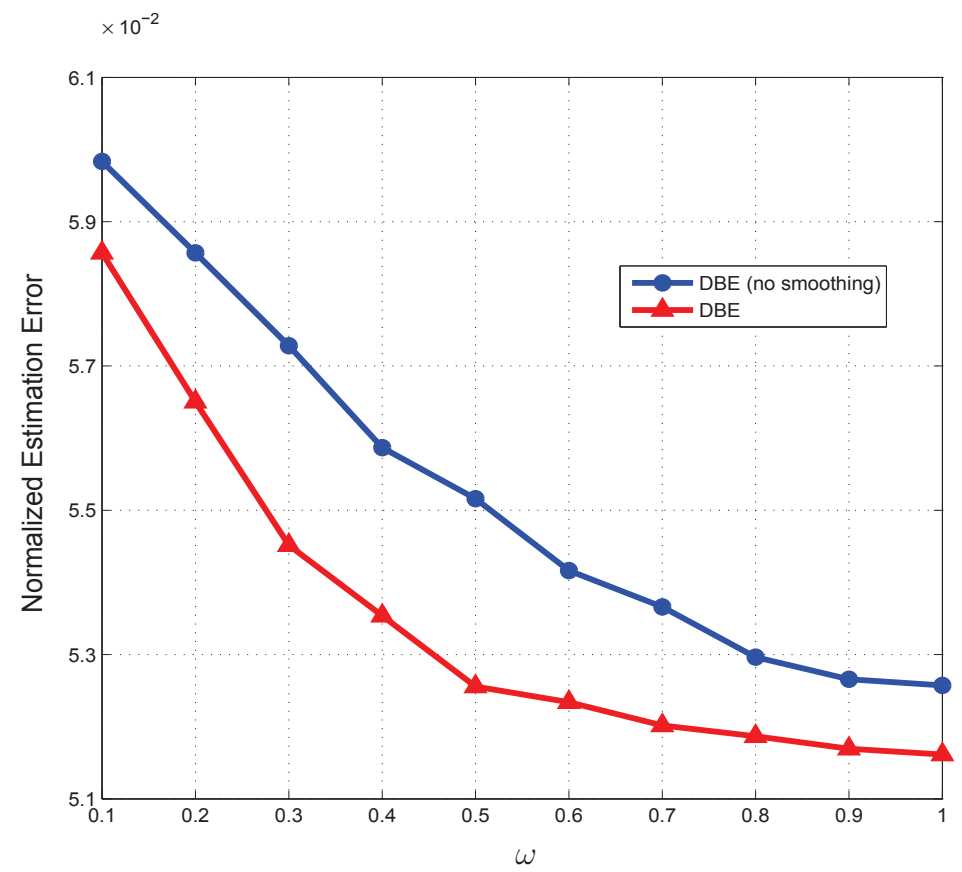

Figure 3-10: Robustness comparison of DBE algorithm with and without smoothing constraints after changing the density.

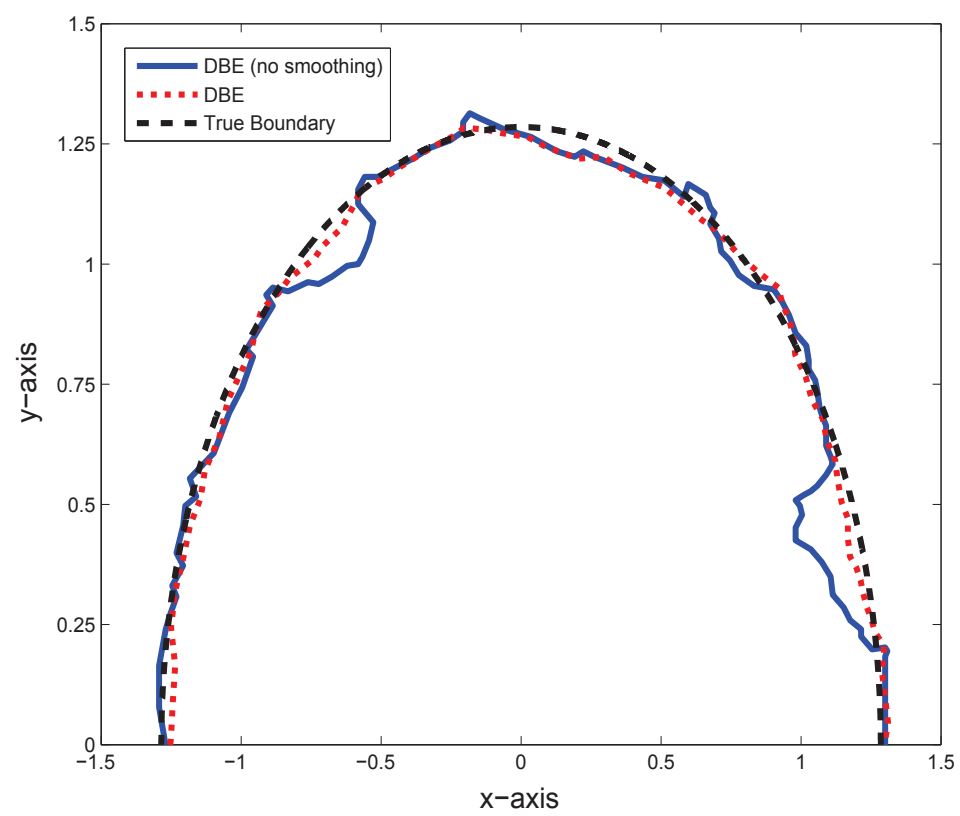

Figure 3-11: Estimated boundaries with and without smoothing constraints. 
algorithm has lower estimation errors and better robustness compared to various other methods.

We have made various simplifying and heuristic assumptions in deriving the optimal SU density. Our simulations however shows that despite these assumptions, the theoretical optimal SU density found is not significantly different from the actual one. Bounding the estimation error more precisely remains a difficult open problem. 
THIS PAGE INTENTIONALLY LEFT BLANK 


\section{Chapter 4}

\section{Channel Allocation and Learning For}

\section{Temporal-Spatial Spectrum Reuse}

In Chapter 3, we have proposed and analyzed a boundary learning method, which could exploit opportunistic temporal-spatial spectrum access without the secondary users having a priori knowledge of the primary user's coverage region. Within the no-talk region of the PU, SUs who do not interfere with each other can make use of the same PU channel. In this chapter, we will propose and analyze several learning polices that enable SUs to identify temporal-spatial spectrum access opportunities within the no-talk region of the PU, without knowing prior knowledge of the primary user's channel idle probabilities.

If SUs are constrained to using different channels at the same time due to interference between them, as assumed in [30,31], then the optimal allocation is to assign each SU a different channel with the best availability. However, spatially separated SUs can share the same channel without significant interference with each other. For 


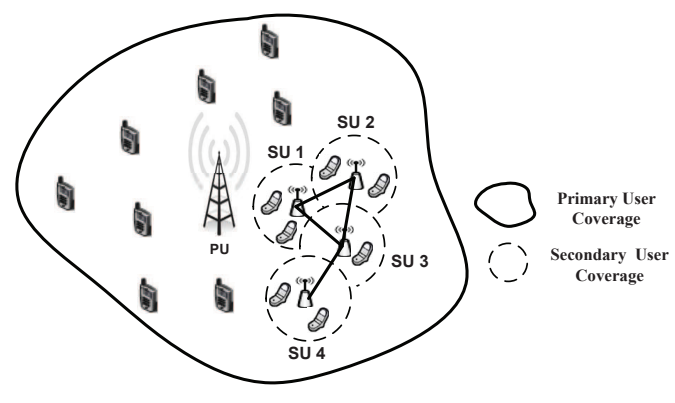

(a) An edge between two SUs indicate that they interfere with each other. When a channel is sensed idle, SUs 1, 2 and 3 cannot use it simultaneously. However, SUs 1 and 4 can reuse the same channel.

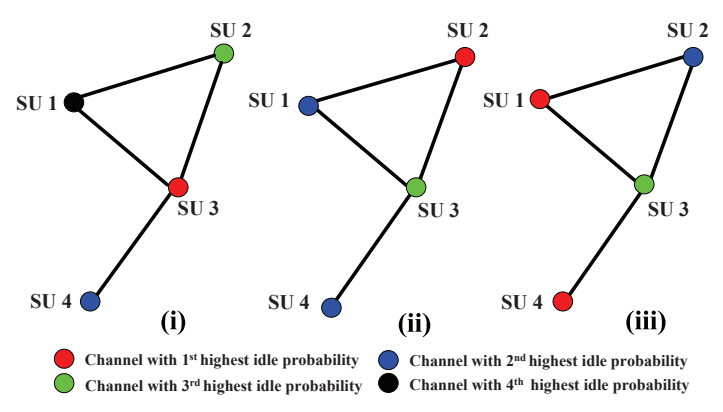

(b) Three possible channel allocations.

Figure 4-1: Spatial spectrum reuse in a CRN with multiple SUs.

example, consider the scenario depicted in Figure 4-1, where the expected network reward is given by the expected total number of interference-free channel uses by the SUs. An edge between two SUs indicates that they interfere with each other. Because SU 1 and SU 4 do not interfere with each other, they can be assigned the same PU channel with the highest idle probability. It can be shown that scenario (iii) in Figure 4-1(b) achieves the highest expected network reward. To the best of our knowledge, this is the first work to consider temporal-spatial spectrum reuse in a MAB formulation.

In this chapter, we firstly propose a centralized policy that uses a central processor to optimize the channel access ranks of the SUs at exponentially increasing time intervals, based on the idle probability estimates of an arbitrary SU. SUs then perform a local random $\epsilon$-greedy channel learning algorithm. We call this the Centralized Channel Allocation (CCA) policy. To overcome the high computation complexity at the central processor in the CCA policy, we then propose two distributed learning policies called the Collaborative Access Ranking and Learning (CARL) policy and 
the Distributed Access Rank Learning (DARL) policy, which could find the channel access ranks and learn the channel statistics. We compare our policies with the random access policy [30], the time-division fair sharing (TDFS) policy [31] and the adaptive randomization policy [30]. Our simulation results suggest that our policies perform significantly better in terms of average regret than the benchmark policies.

The rest of this chapter is organized as follows. In Section 4.1, we introduce our system model and problem formulation. We present a centralized channel allocation policy in Section 4.2. We then propose two distributed channel access ranking and learning pollices, and derive theoretical bounds on the regret achieved by the two polices in Section 4.3. In Section 4.4, we show simulation results and we conclude this chapter in Section 4.5.

\subsection{Problem Formulation}

Suppose that there are $M \geq 2$ secondary users and $N$ orthogonal channels in a CRN. We model the SU network as a graph $G=(V, E)$, where $V$ is a set of independent and identically distributed SUs, and $E$ is a set of edges. Two SUs are connected by an edge if the mutual interference between them is above a predefined threshold. If two SUs are not connected via an edge, then we assume that they can utilize the same PU channel simultaneously.

Let $\mathcal{N}$ be a set of channels and $\mathcal{M}$ be a set of SUs. We divide time into equal intervals. In each time slot $n$, each channel $j \in \mathcal{N}$ is idle with probability $\mu_{j} \in(0,1)$, independent of all other channels. Without loss of generality, we assume that $\mu_{1}>$ 
$\mu_{2} \geq \mu_{3} \geq \ldots \geq \mu_{N}$ (SUs are not aware of this ordering). For each $j \in \mathcal{N}$, we use $S_{j}(n)$ to denote the channel state of a channel $j$ in time slot $n$ with $S_{j}(n)=1$ if the channel $j$ is idle and 0 otherwise.

To simplify our formulation and algorithm descriptions, we include a sufficient number of null channels each with idle probability zero and let $\mathcal{N}^{+}$be the set of channels $\mathcal{N}$ augmented with the null channels such that $\left|\mathcal{N}^{+}\right| \geq M$. A SU allocated a null channel simply means that it is not able to perform OSA in practice. This allows us to include cases where there are insufficient number of PU channels to be allocated to all the SUs.

Since the channel idle probabilities are unknown to all SUs, each SU needs to learn them through their sensing observations. In each time slot $n$, each SU can only sense and access one channel. Let $X_{i, j}(n)=1$ if SU $i$ chooses channel $j$ and senses that it is idle, and $X_{i, j}(n)=0$ otherwise. We assume that channel sensing is perfect for all SUs so that $X_{i, j}(n)=S_{j}(n)$ if channel $j$ is chosen by SU $i$ at time slot $n$.

Let $Y_{i, j}(n)$ be the reward of a SU $i$ from accessing a channel $j \in \mathcal{N}^{+}$in slot $n$ after sensing it free. Let $\mathcal{M}_{i}$ be the set of neighboring SUs of the $\mathrm{SU} i$ in the graph $G$, not including SU $i$ itself. If any SU $k \in \mathcal{M}_{i}$ uses the same channel as that of SU $i$, packet collisions occur. We adopt the following reward model:

$$
Y_{i, j}(n)= \begin{cases}1 & \text { if channel } j \text { is idle and no other } k \in \mathcal{M}_{i} \\ & \text { transmits over it in the same time slot } n \\ 0 & \text { otherwise. }\end{cases}
$$


This reward model is similar to one used in [30,31], the only difference being that $\mathcal{M}_{i}=\mathcal{M}$ for all $i$ in that model, i.e., every $\mathrm{SU}$ interferes with each other when using the same channel.

We are interested to design a policy $\psi$ to learn the channel idle probabilities so as to maximize the total expected number of successful transmissions of all SUs by exploiting spatial channel reuse among SUs. The policy $\psi$ is a rule that determines which channel $\psi_{i}(n) \in \mathcal{N}$ SU $i$ chooses to sense in time slot $n$. The choice $\psi_{i}(n)$ can be made based on the sensing results of SU $i$ at previous time slots $1,2, \ldots, n-1$, and on the previous channel choices $\left\{\psi_{i}(l):\right.$ for $\left.l<n\right\}$. If the channel $\psi_{i}(n)$ is idle, $\mathrm{SU} i$ will transmit over the channel. At the end of each time slot $n$, each $\mathrm{SU}$ is assumed to know whether it has transmitted successfully or not (e.g., through an acknowledgment from the SU receiver). Let $T_{i, j}(n)$ be the total number of time slots that the $\mathrm{SU} i$ has sensed the channel $j$ in $n$ time slots, and let $V_{i, j}(n)=\sum_{l \leq n} Y_{i, j}(l)$ be the total number of time slots that the channel $j$ is successfully accessed by $\mathrm{SU} i$ up to time slot $n$. After $n$ time slots, the total network reward is $\sum_{i \in \mathcal{M}} \sum_{j \in \mathcal{N}^{+}} \mu_{j} \mathbb{E}\left[V_{i, j}(n)\right]$, which is proportional to the network throughput.

The regret of the policy $\psi$ until time slot $n$ is defined as the difference between the total reward of a genie-aided rule and the expected reward of all SUs given by

$$
R(n, \psi)=n \sum_{i \in \mathcal{M}} \mu_{\pi^{*}(i)}-\sum_{i \in \mathcal{M}} \sum_{j \in \mathcal{N}^{+}} \mu_{j} \mathbb{E}\left[V_{i, j}(n)\right]
$$

where $\pi^{*}: \mathcal{M} \mapsto \mathcal{N}^{+}$is an optimal channel allocation if all channel idle probabilities are known. Let $x_{i j}^{*}$ be an indicator variable with value $1 \mathrm{iff} \pi^{*}(i)=j$, i.e., $x_{i j}^{*}=1 \mathrm{iff}$ 
$\mathrm{SU} i$ is allocated channel $j$. Then $\left(x_{i j}^{*}\right)_{i \in \mathcal{M}, j \in \mathcal{N}^{+}}$is an optimal solution to the following optimization problem:

$$
\begin{array}{ll}
\text { (P0) } \max _{x_{i j}} & \sum_{i \in \mathcal{M}} \sum_{j \in \mathcal{N}^{+}} x_{i j} \mu_{j} \\
\text { s.t. } \quad & x_{i j}+\sum_{k \in \mathcal{M}_{i}} x_{k j} \leq 1, \forall i \in \mathcal{M}, j \in \mathcal{N}^{+}, \\
& \sum_{j \in \mathcal{N}^{+}} x_{i j} \leq 1, \forall i \in \mathcal{M}, \\
& x_{i j} \in\{0,1\}, \forall i \in \mathcal{M}, j \in \mathcal{N}^{+} .
\end{array}
$$

In the above optimization problem, $x_{i j}$ is an indicator variable that takes value one if channel $j$ is allocated to SU $i$. The constraint (4.4) ensures that neighboring SUs do not use the same channel, while the constraint (4.5) ensures that each SU is allocated at most one PU channel.

The above maximization problem is an integer linear program, which corresponds to a NP-complete decision problem (of which finding if there exists an independent set in the graph $G$ for a given size is a special case [131]). In general, even if the channel idle probabilities are known a priori, it is analytically difficult for a genie to find the optimal channel allocations. To ensure that optimization is done within a reasonable amount of time, the genie can adopt an approximate method [132], which however leads to a linear regret as the number of time slots $n \rightarrow \infty$. For a distributed policy that does not know the channel idle probabilities a priori, the problem is even harder, and in general we cannot hope to learn the channel probabilities and an optimal channel allocation with sub-linear regret, unlike other MAB problems in 
which logarithmic regrets are common [30,31].

For any channel allocation $\pi: \mathcal{M} \mapsto \mathcal{N}^{+}$, we say that $\pi(i)$ is the channel access rank of SU $i$ because of the assumption that $\mu_{1}>\mu_{2} \geq \ldots \geq \mu_{N}$. Our main idea is to learn the optimal channel access rank of each SU and the idle probability of each channel in order to optimize the regret. In the following, we propose a centralized policy that we show to have asymptotic logarithmic regret, but requires solving an analytically difficult optimization problem like (P0) at exponentially increasing time intervals. We also propose heuristic distributed policies under an additional technical assumption. These heuristic policies have linear regret in general. We compare our distributed policies to those in $[30,31]$ when applied to an incomplete graph $G$, and show that those policies also have linear regrets since they do not consider spatial reuse of channels. Our simulation results however indicate that our distributed policies have smaller regret than the existing ones in [30,31].

For the convenience of the reader, we list some Chapter 4 commonly used notations in Table 4.1. Some of the notations have been defined in this section, while the remaining ones will be defined formally in the sequel where they first appear. In addition, for non-negative functions $f$ and $g$, we say that $f(n) \in O(g(n))$ if $\limsup _{n \rightarrow \infty} f(n) / g(n)<\infty$, $f(n) \in \Omega(g(n))$ if $\liminf _{n \rightarrow \infty} f(n) / g(n)>0$, and $f(n) \in \Theta(g(n))$ if $f(n) \in O(g(n))$ and $f(n) \in \Omega(g(n))$. The notation $\mathcal{P}(A)$ is the probability of the event $A$. 
Table 4.1: Summary of notations used in Chapter 4

\begin{tabular}{|c|c|}
\hline Symbol & Definition \\
\hline$M$ & number of SUs \\
\hline$N$ & number of channels \\
\hline $\mathcal{M}$ & set of SUs \\
\hline $\mathcal{N}$ & set of channels \\
\hline$\mu_{j}$ & idle probability of channel $j$ \\
\hline$X_{i, j}(n)$ & sensing decision of SU $i$ for channel $j$ in time slot $n$ \\
\hline$Y_{i, j}(n)$ & reward of SU $i$ from accessing channel $j$ in time slot $n$ \\
\hline$R(n, \psi)$ & regret of policy $\psi$ until time slot $n$ \\
\hline$r_{i}(n)$ & channel access rank of SU $i$ in time slot $n$ \\
\hline$\rho_{i}(n)$ & channel identity sensed by SU $i$ in time slot $n$ \\
\hline P0 & channel allocation optimization problem 4.3 \\
\hline$t_{k}$ & $k$-th time instance to solve $(\widehat{\mathrm{P} 0})$ \\
\hline$\xi(n)$ & number of time instances to solve $(\widehat{\mathrm{P} 0})$ within $n$ time slots \\
\hline$l_{k}$ & number of time slots between $t_{k+1}$ and $t_{k}$ \\
\hline$\gamma$ & parameter in channel learning algorithm \\
\hline$\delta$ & parameter in channel learning algorithm \\
\hline$\overline{\epsilon_{n}}$ & $\begin{array}{l}\text { probability to sense a channel } j \in \mathcal{N} \text { based on } \gamma \text { and } \delta \\
\text { in time slot } n\end{array}$ \\
\hline$\chi(G)$ & chromatic number of the graph $G$ \\
\hline$I_{j}$ & maximal independent set $j$ of the graph $G$ \\
\hline$d(i)$ & degree of SU $i$ \\
\hline$u(i)$ & palette of forbidden colors for $\mathrm{SU} i$ \\
\hline$\triangle(G)$ & maximum vertex degree \\
\hline
\end{tabular}

\subsection{Centralized Channel Allocation Policy}

In this section, we propose a centralized policy $\psi^{\mathrm{CCA}}$, and show that it has asymptotic $\log$ regret.

We assume that there is a central processor in the CRN capable of solving problem $(\widehat{\mathrm{P} 0})$ with the true channel idle probabilities $\mu_{j}, j=1, \ldots, N$, replaced by empirical estimates from an arbitrary SU. We call this optimization problem $(\widehat{\mathrm{P} 0})$. However, 
since $(\widehat{\mathrm{P} 0})$ corresponds to a NP-complete decision problem, we suppose that the central processor only performs this optimization at specific irregular time instances (see Figure 4-2) instead of at every time slot. For a time horizon $n$, let $t_{k}, k=1, \ldots, \xi(n)$, be the $\xi(n)$ time instances at which the central processor solves ( $\widehat{\mathrm{P} 0})$ with updated empirical estimates of $\mu_{j}, j=1, \ldots, N$. We assume $t_{1}<\infty$, i.e., there is at least one optimization time instance.

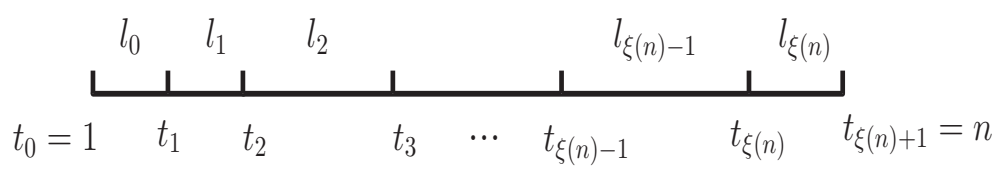

Figure 4-2: Optimization time instances for $\psi^{\mathrm{CCA}}$.

For $k=0, \ldots, \xi(n)$, let $l_{k}=t_{k+1}-t_{k}-1$, where $t_{0}=1$ and $t_{\xi(n)+1}=n$, be the number of time slots starting from the $k$-th optimization, and before the next optimization by the central processor. Let $\bar{X}_{i, j}(n)=\sum_{k=1}^{n} X_{i, j}(k) / T_{i, j}(n)$ be the empirical estimate of the idle probability of channel $j$ by SU $i$.

At each time instance $t_{k}, k=1, \ldots, \xi(n)$, an arbitrarily chosen SU $i$ sends $\left\{\bar{X}_{i, j}\left(t_{k}\right): j \in \mathcal{N}\right\}$ to the central processor, which replaces $\mu_{j}$ with $\bar{X}_{i, j}\left(t_{k}\right)$ in problem (P0), and finds the optimal or near-optimal solution using the branch and bound algorithm [132]. Let $\left\{r_{i}\left(t_{k}\right): i \in \mathcal{M}\right\}$ be the optimal channel access ranks found by the central processor (i.e., $r_{i}\left(t_{k}\right)=j$ iff $x_{i j}=1$ in the solution of $(\widehat{\mathrm{P} 0})$ ). These ranks are then communicated to the SUs, which utilizes their assigned ranks in a local random $\epsilon$-greedy channel learning algorithm: In each time slot of the channel learning period, each SU $i$ chooses to sense a channel $j \in \mathcal{N}$ (not $\mathcal{N}^{+}$since there is no need to learn the null channels) with probability $\epsilon_{t}$, and with probability $1-\epsilon_{t}$ chooses the $r_{i}\left(t_{k}\right)$-th 
best channel according to its empirical idle probability estimates $\left\{\bar{X}_{i, j}\left(t_{k}\right): j \in \mathcal{N}^{+}\right\}$. Let $\rho_{i}(t)$ be the channel chosen by $\mathrm{SU} i$ in time slot $t$. This learning algorithm is an extension of the work in [29]. The probability $\epsilon_{t}$ is chosen to be decreasing in $t$ with a specific form as shown in Algorithm 4.1. To do this, we need the following assumption.

Assumption 1. A positive lower bound $\Delta_{1} \leq \min _{1 \leq j<N}\left|\mu_{j}-\mu_{j+1}\right|$ is known to the SUs.

Note that for Assumption 1 to hold, SUs do not need to know the channel idle probabilities, but an estimate of how different the PU channel utilization rates are from each other. In practice, one can always choose a sufficiently small $\Delta_{1}$.

We call the above procedure the Centralized Channel Allocation Policy $\psi^{\mathrm{CCA}}$, and its formal description is given in Algorithms 4.1 and 4.2. Note that if for some $i \in \mathcal{M}$, the central processor returns $r_{i}\left(t_{k}\right)>N$, then $\mathrm{SU} i$ is allocated a null channel and it does not engage in OSA for $t \in\left[t_{k}, t_{k+1}\right)$.

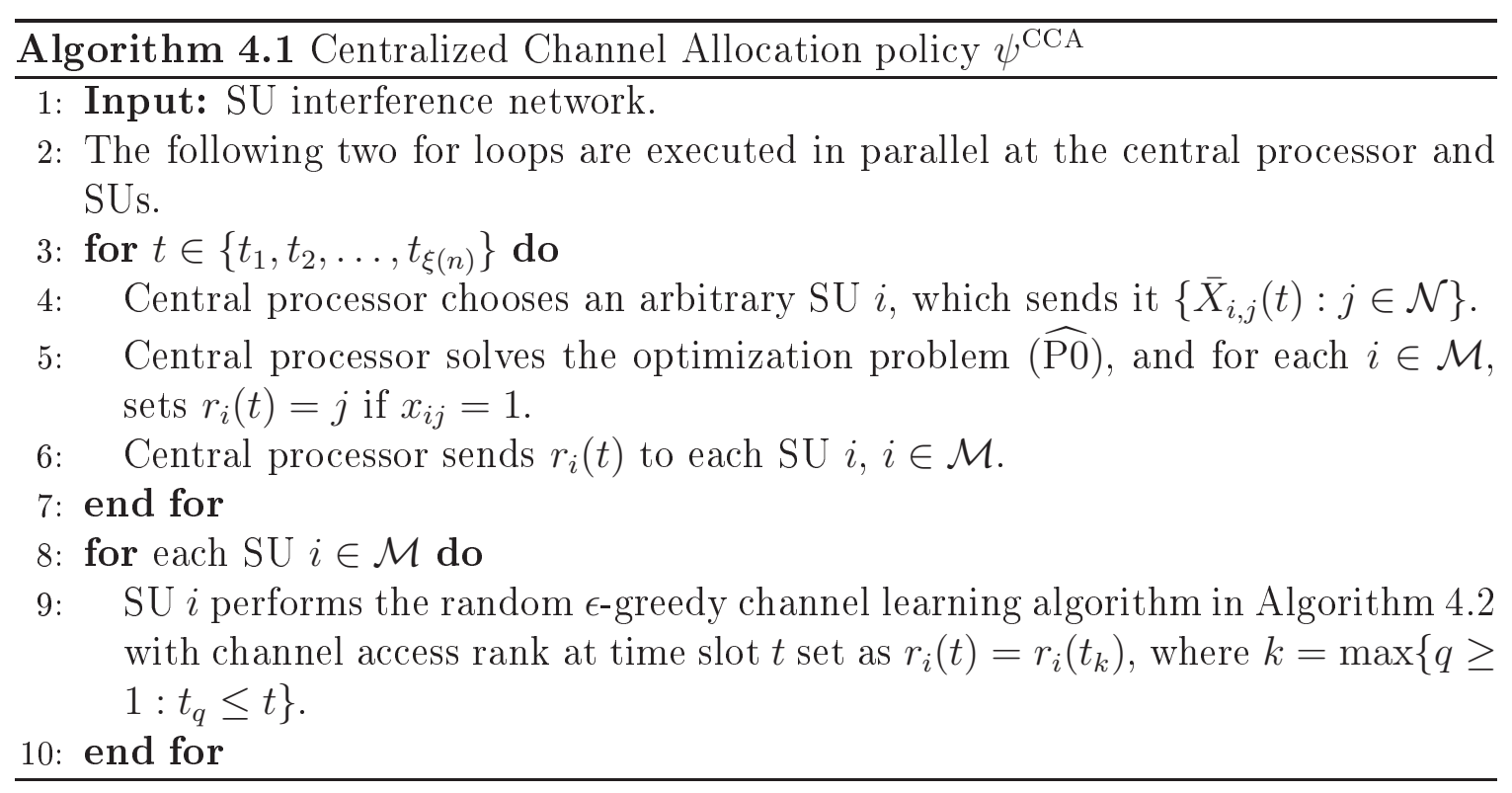




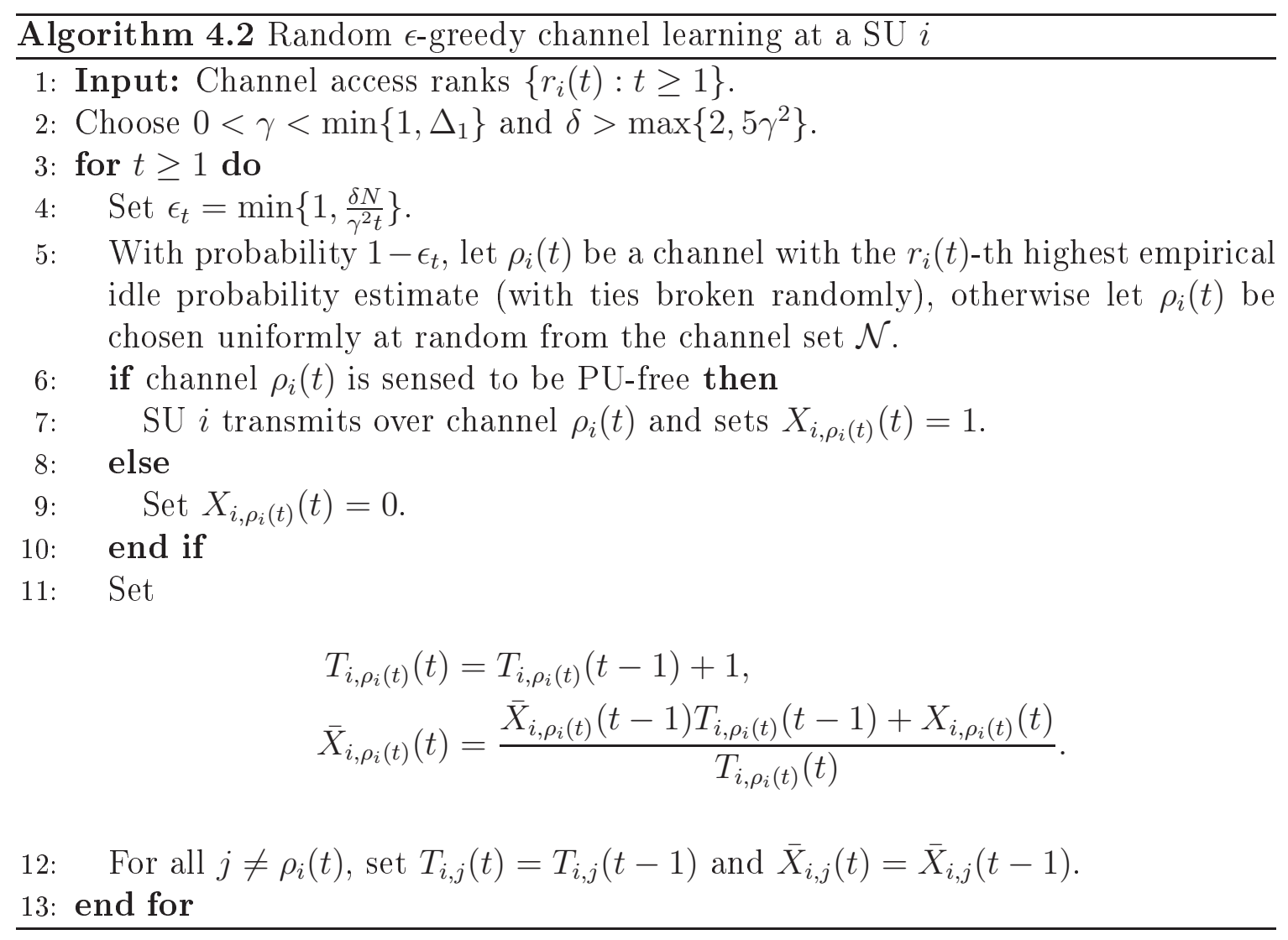

We first show that for any policy $\psi$, the regret is at least $\Omega(\log n)$.

Proposition 4.1. For any policy $\psi, R(n, \psi) \in \Omega(\log n)$.

Proof. If two SUs use the same channel in the same time slot, a collision occurs and both SUs have rewards of 0 . Therefore, $R(n, \psi) \geq R^{\prime}(n)$, where $R^{\prime}(n)$ denotes the regret if SUs are still rewarded with a reward of 1 even if collisions occur. This regret $R^{\prime}(n)$ is then equivalent to $M$ times the regret of a single-user MAB problem, which has been shown to be $\Omega(\log n)$ in [30].

The next result shows that the regret using $\psi^{\mathrm{CCA}}$ is order-optimal for appropriately chosen optimization time instances. 
Theorem 4.1. If $l_{k}>l_{k-1}$ for $1<k<\xi(n)$ and $l_{k} \leq c l_{k-1}$ for all $k \geq 2$ and some $c>0$, then $R\left(n, \psi^{\mathrm{CCA}}\right) \in \Theta(\log n)$.

Proof. The following lemma can be shown using the exact same argument in Theorem 3 of [29], and its proof is thus omitted here.

Lemma 4.1. Suppose $\delta>5$ and $0<\gamma<\min \{1, \Delta\}$ for some $\Delta>0$ in Algorithm 4.2. Then, for all $i \in \mathcal{M}, n \geq \frac{\delta N}{\gamma}$ and $j \in \mathcal{N}$, we have

$$
\mathcal{P}\left(\left|\bar{X}_{i, j}(n)-\mu_{j}\right| \geq \frac{\Delta}{2}\right) \leq \frac{a}{n^{1+\varepsilon}}
$$

where $a$ and $\varepsilon$ are positive constants.

Let $R(k)$ be the total regret under $\psi^{\mathrm{CCA}}$ in the time slots $\left[t_{k}, t_{k+1}\right)$. Let $A\left(t_{k}\right)=$ $\left\{r_{i}\left(t_{k}\right)=\pi^{*}(i), \forall i \in \mathcal{M}\right\}$ be the event that the central processor returns the optimal channel allocation at time $t_{k}$. Then, for each time $m \in\left[t_{k}, t_{k+1}\right)$, conditioned on $A\left(t_{k}\right)$, a packet collision occurs only if for some $i \in \mathcal{M}$, either $\left|\bar{X}_{i, \pi^{*}(i)}(m)-\mu_{\pi^{*}(i)}\right| \geq \Delta_{1} / 2$ or $\mathrm{SU} i$ chooses a random channel $\rho_{i}(m) \neq \pi^{*}(i)$. On the other hand, the event $A^{c}\left(t_{k}\right)$ occurs only if $\left|\bar{X}_{i, \pi^{*}(i)}\left(t_{k}\right)-\mu_{\pi^{*}(i)}\right| \geq \Delta_{2} / 2$ for some $i \in \mathcal{M}$, where $\Delta_{2}$ is the minimum sensitivity range of the $\mu_{j}$ coefficients, $j=1, \ldots, N$, in (P0) with respect to the optimal solution. Note that $\Delta_{2}>0$ since (4.3) is a continuous function. Therefore, we have

$$
\begin{aligned}
R(k) & \leq \mu_{1} \sum_{m=t_{k}}^{t_{k+1}-1}\left(\mathcal{P}\left(A\left(t_{k}\right) \text { and }\left\{\left|\bar{X}_{i, \pi^{*}(i)}(m)-\mu_{\pi^{*}(i)}\right| \geq \frac{\Delta_{1}}{2} \text { or } \rho_{i}(m) \neq \pi^{*}(i)\right\}\right)+\mathcal{P}\left(A^{c}\left(t_{k}\right)\right)\right) \\
& \leq \mu_{1} \sum_{m=t_{k}}^{t_{k+1}-1} \sum_{i \in m}\left(\mathcal{P}\left(\left|\bar{X}_{i, \pi^{*}(i)}(m)-\mu_{\pi^{*}(i)}\right| \geq \frac{\Delta_{1}}{2}\right)+2 \epsilon_{m}\right)
\end{aligned}
$$




$$
+\mu_{1} \sum_{m=t_{k}}^{t_{k+1}-1} \sum_{i \in m} \mathcal{P}\left(\left|\bar{X}_{i, \pi^{*}(i)}(m)-\mu_{\pi^{*}(i)}\right| \geq \frac{\Delta_{2}}{2}\right)
$$

where the last inequality follows from the union bound. From Lemma 4.1, the right hand side of (4.7) is upper-bounded by

$$
\begin{aligned}
& c_{1}\left(\sum_{m=t_{k}}^{t_{k+1}-1}\left(\frac{1}{m^{1+\epsilon}}+\frac{1}{m}\right)+\sum_{m=t_{k}}^{t_{k+1}-1} \frac{1}{m^{1+\epsilon}}\right) \\
& \leq c_{2} \frac{l_{k}}{t_{k}},
\end{aligned}
$$

for some constants $c_{1}$ and $c_{2}$ sufficiently large. Therefore, we have for some constant $c_{3}>0$,

$$
\begin{aligned}
R\left(n, \psi^{\mathrm{CCA}}\right) & \leq c_{3} t_{1}+c_{2} \sum_{k=1}^{\xi(n)} \frac{l_{k}}{t_{k}} \\
& \leq c_{3} t_{1}+c_{2} \sum_{k=1}^{\xi(n)} \frac{l_{k}}{l_{k-1}} \\
& \leq c_{3} t_{1}+c_{2} c \xi(n),
\end{aligned}
$$

where the second inequality follows because $t_{k}=\sum_{m=0}^{k-1} l_{m} \geq l_{k-1}$. Since $l_{k}>l_{k-1}$ for $1<k<\xi(n)$ and $l_{k} \leq c l_{k-1}$ for all $k \geq 2$, we have $\xi(n) \leq c_{4} \log (n)$ for some constant $c_{4}>0$. Then from (4.8), we have $R\left(n, \psi^{\mathrm{CCA}}\right) \in O(\log n)$, and together with Proposition 4.1 that $R\left(n, \psi^{\mathrm{CCA}}\right) \in \Omega(\log n)$, the theorem follows.

Theorem 4.1 shows that the central processor needs to perform a re-optimization of $(\widehat{\mathrm{P} 0})$ only at exponentially increasing time intervals. It also shows that our proposed $\psi^{\mathrm{CCA}}$ is an order optimal policy. However, since $(\widehat{\mathrm{P} 0})$ is a NP-complete problem, the 
central processor will incur high computation cost at each optimization time instance if the number of SUs is large. Furthermore, the CRN is also vulnerable to the failure of the central processor. In the next section, we propose heuristic distributed policies that do not have such drawbacks, but which are no longer order optimal.

\subsection{Distributed Channel Access Ranking and Learn- ing}

In this section, we propose two heuristic distributed learning policies to learn channel access ranks and channel statistics .

We know that finding the optimal channel access ranking is still NP-complete. However, the genie-aided channel allocation in (P0) could become a graph coloring problem in which we wish to partition the graph into disjoint maximal independent sets $I_{1}, \ldots, I_{\chi(G)}$ (shown in non-increasing order), where $\chi(G)$ is known as the chromatic number of the graph $G$ [133]. The SUs assigned to the same independent set are allocated the same channel, with a larger independent set being assigned a channel with a higher idle probability. Therefore, we seek to order the independent sets according to their sizes, with a larger set assigned a lower channel access rank (ties are broken arbitrarily). To simplify the descriptions and analysis of our proposed algorithms, we assume the channel number $N \geq \chi(G)$, i.e., each independent set could 
be allocated a different channel. The regret (4.2) can then be equivalently written as

$$
R(n, \psi)=n \sum_{j=1}^{\chi(G)} \mu_{j}\left|I_{j}\right|-\sum_{i \in \mathcal{M}} \sum_{j \in \mathcal{N}^{+}} \mu_{j} \mathbb{E}\left[V_{i, j}(n)\right]
$$

where $\left|I_{j}\right|$ is the number of SUs in the maximal independent set $I_{j}$.

Our first policy, which we call Collaborative Access Ranking and Learning (CARL), is a three-stage distributed channel access ranking and learning policy, which however incurs overheads in SU synchronization. The second policy, which we call Distributed Access Rank Learning (DARL), does not require SU synchronization, but is expected to have higher regret than CARL. We also derive performance bounds for both policies.

\subsubsection{CARL Policy}

The CARL policy is a three-stage distributed channel access ranking and learning policy denoted by $\psi^{\mathrm{CARL}}$, and which performs the following procedures:

1. distributed graph coloring algorithm (Algorithm 4.3);

2. distributed channel access rank determination method (Algorithm 4.4); and

3. random $\epsilon$-greedy channel learning (Algorithm 4.2).

The first two stages enable each SU to find its optimal channel access rank, which will be utilized in the last stage to find the optimal channel allocation for maximizing the total network reward. 
The graph coloring algorithm is described in Algorithm 4.3, which aims to cluster SUs into a minimal number of maximal independent sets so that channels with higher idle probabilities are spatially reused by more SUs. We adopt a distributed greedy graph coloring algorithm $[134,135]$ to color the graph $G$ using the smallest number of colors. Suppose that SUs are colored using a set of colors $\left\{1, \ldots,\left|\mathcal{N}^{+}\right|\right\}$, with SUs labeled the same color belonging to the same maximal independent set. In the graph $G$, let $d(i)$ denote the degree of SU $i$ and $u(i)$ be the palette of forbidden colors, which are used by its colored neighbors. Let $\alpha(i)=|u(i)|$ be the number of different colors used by the SUs in the neighborhood of SU $i$. A color is said to be legal for SU $i$ if it is not contained in $u(i)$. In each round, each SU generates a random value $\lambda_{i}$ and the $\mathrm{SU}$ with the highest $\alpha(i)$ will be colored. If two SUs have the same $\alpha(i)$, the $\mathrm{SU}$ with a higher $\lambda_{i}$ will be colored first.

The graph coloring algorithm in Algorithm 4.3 is heuristic and is not guaranteed to find an optimal coloring for a general graph. It can use up to $\triangle(G)+1$ colors to color a general graph, where $\triangle(G)$ is the maximum vertex degree. However, in practice, CRNs are usually sparse satisfying $\triangle(G)+1 \ll M \leq N$ and Algorithm 4.3 has been shown to find the chromatic number of such graphs effectively [135]. Furthermore, it produces optimal colorings for complete graphs and bipartite graphs like trees [136]. Since in each time slot, at least one uncolored node in the graph is colored, Algorithm 4.3 requires at most $O(M)$ rounds to color all nodes. In each round, at most $O\left(M^{2}\right)$ messages are exchanged among nodes so that the message complexity of Algorithm 4.3 is $O\left(M^{3}\right)$.

After performing the distributed graph coloring step, SUs are clustered into dif- 


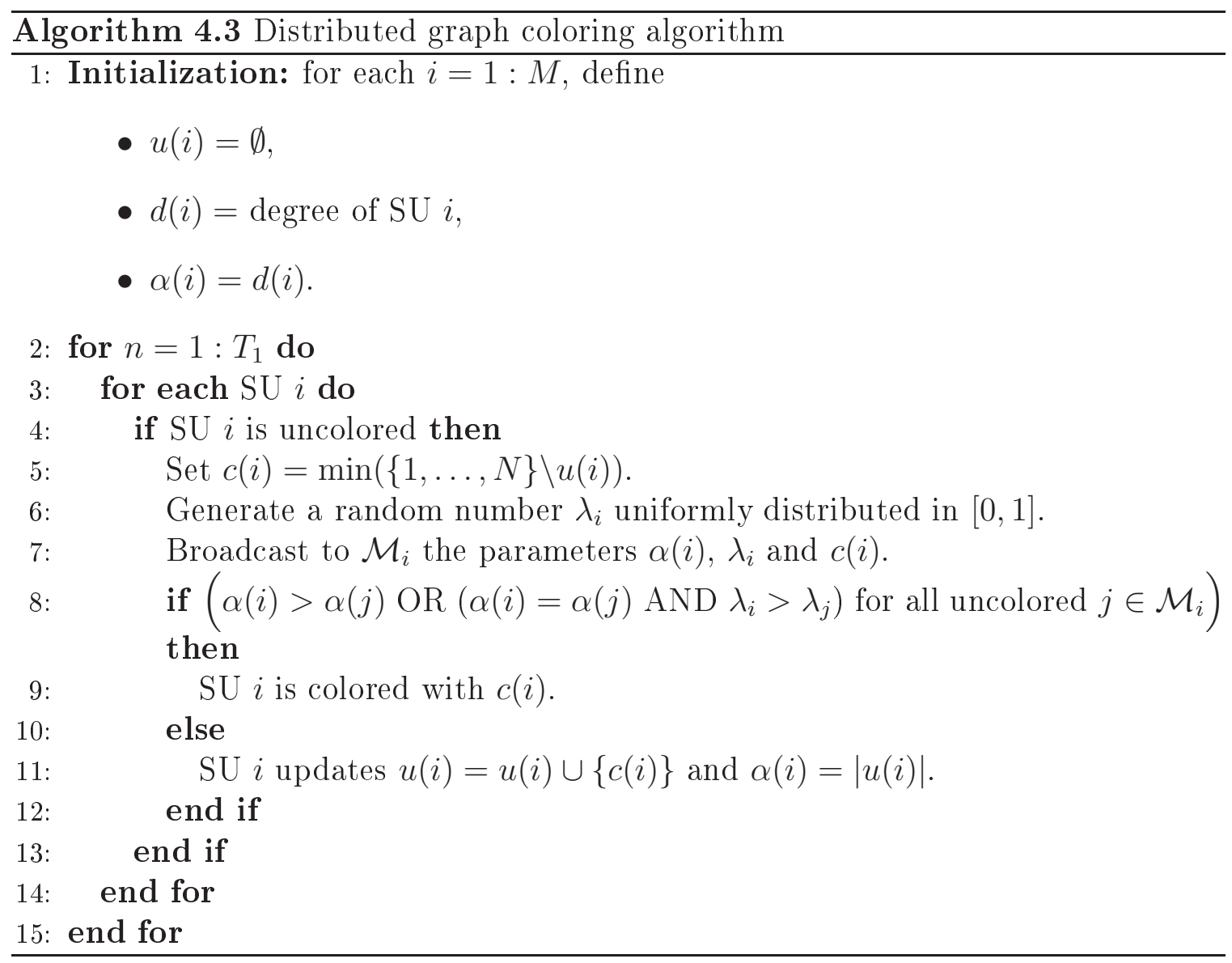

ferent color groups identified by $c(i) \in\left\{1, \ldots,\left|\mathcal{N}^{+}\right|\right\}$for each $i \in \mathcal{M}$. To maximize the total network reward, the group having more SUs should be assigned a smaller channel access rank so that more SUs can access a PU channel with higher idle probability. Therefore, we need to relabel the group identities according to their group sizes. In each time slot $n$, each $\mathrm{SU} i$ maintains two variable vectors $\mathbf{z}_{i, n}=\left[z_{i, n}(1), \ldots, z_{i, n}\left(\left|\mathcal{N}^{+}\right|\right)\right]$and $\mathbf{w}_{i, n}$, where we initialize $\mathbf{w}_{i, 1}=\mathbf{z}_{i, 1}$, and set $z_{i, 1}(c(i))=1$ and all other entries in $\mathbf{z}_{i, 1}$ to 0 . We use an average consensus algorithm [137] to compute $\overline{\mathbf{z}}=\sum_{i=1}^{M} \mathbf{z}_{i, 1} / M$ in a distributed manner (see Algorithm 
4.4). From the time slot $n>1$, each $\mathrm{SU} i$ updates $\mathbf{z}_{i, n}$ and $\mathbf{w}_{i, n}$ according to:

$$
\begin{aligned}
& \mathbf{w}_{i, n+1}=\mathbf{z}_{i, n}+\frac{1}{2} \sum_{j \in \mathcal{M}_{i}} \frac{\mathbf{z}_{j, n}-\mathbf{z}_{i, n}}{\max (d(i), d(j))}, \\
& \mathbf{z}_{i, n+1}=\mathbf{w}_{i, n+1}+\left(1-\frac{2}{9 M+1}\right)\left(\mathbf{w}_{i, n+1}-\mathbf{w}_{i, n}\right) .
\end{aligned}
$$

By ordering the entries of $\mathbf{w}_{i, n}$ at the end of Algorithm 4.4, each SU $i$ can then determine its channel access rank $r_{i}$.

From Theorem 1.1 in [137], we have

$$
\begin{aligned}
\left\|\mathbf{w}_{i, n}-\overline{\mathbf{z}}\right\|_{2}^{2} & \leq 2\left(1-\frac{1}{9 M}\right)^{n-1}\left\|\mathbf{w}_{i, 1}-\overline{\mathbf{z}}\right\|_{2}^{2} \\
& \leq 2 M\left(1-\frac{1}{9 M}\right)^{n-1}
\end{aligned}
$$

Therefore, to ensure that each SU's local estimate of $\mathbf{w}_{i, n}$ converges to $\overline{\mathbf{z}}$, we can set the upper bound in (4.9) to $1 /(2 M)^{2}$ to obtain an upper bound on the number of iterations $T_{2}$ required for Algorithm 4.4 to be $3 \log (2 M) / \log (9 M /(9 M-1))$.

Then, each SU $i$ independently uses $r_{i}$ from Algorithm 4.4 as the random access rank in $\epsilon$-greedy channel learning process mentioned in Algorithm 4.2

\subsubsection{DARL Policy}

The policy $\psi^{\mathrm{CARL}}$ uses its first two stages to determine the appropriate channel access ranks to assign to each SU. This requires that SUs are synchronized between each of its three stages, and may incur significant communication overhead if the number of 


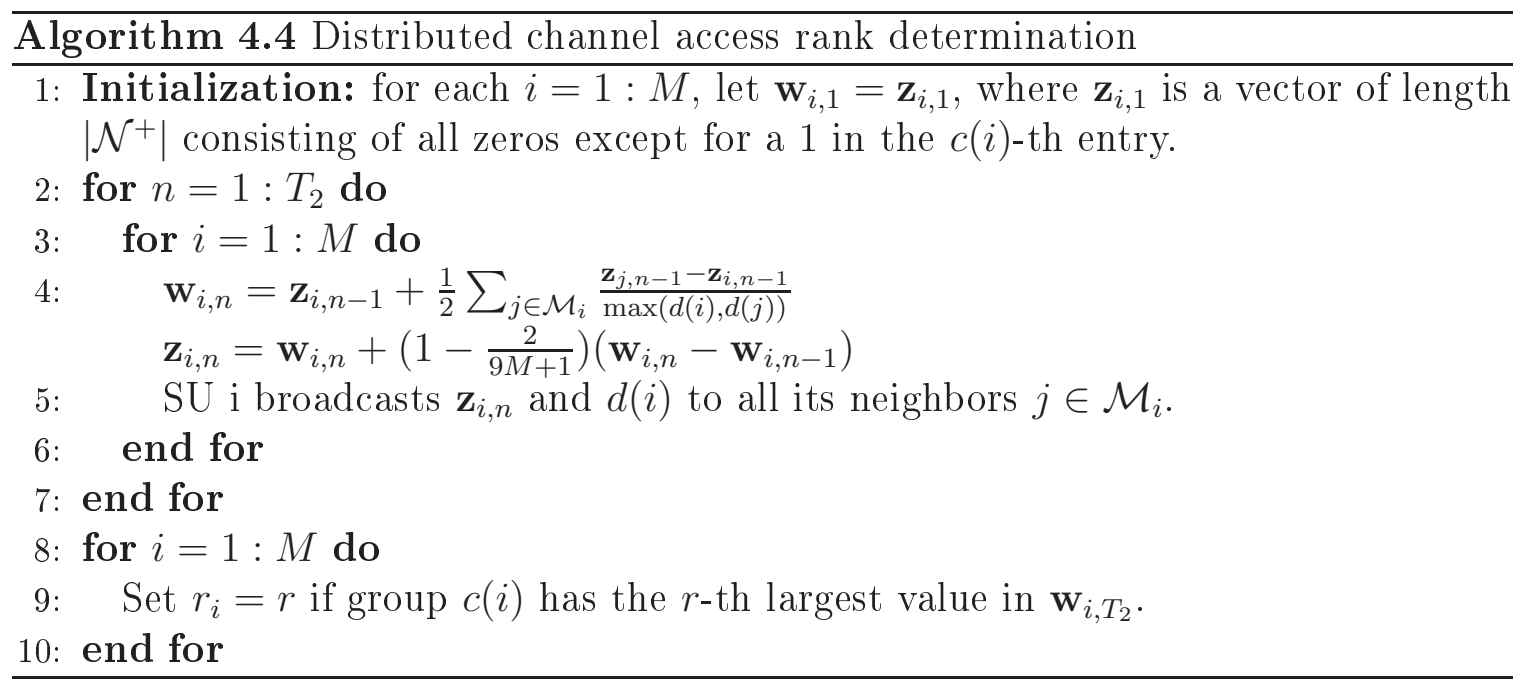

SUs is large. To mitigate this problem, we propose another distributed policy DARL, denoted as $\psi^{\mathrm{DARL}}$, which embeds the channel access rank determination procedure in the channel statistics learning process (see Algorithm 4.5). Since there is a higher likelihood for DARL to assign incorrect channel access ranks to the SUs, we expect DARL to have higher regret than CARL, as verified by the simulation results in Section 4.4.

At the start of DARL, the channel access ranks of SUs $r_{i}(1), i \in \mathcal{M}$ are all set to be 1 . In subsequent time slots $n>1$, if there is no collision for $\mathrm{SU} i$ in the previous time slot, it continues to use the same channel access rank as $r_{i}(n-1)$. Otherwise, it generates a random number $\lambda_{i}$ uniformly distributed in $[0,1]$ and keeps on using the same channel access rank if $\lambda_{i}$ has the largest value among all its neighbors who also have collisions in the previous time slot. If its random number $\lambda_{i}$ is not the largest value, SU $i$ is allocated a channel access rank uniformly and randomly from $\{1, \ldots, N\}$. SU $i$ then performs the $\epsilon$-greedy channel learning process as given in Algorithm 4.2. 


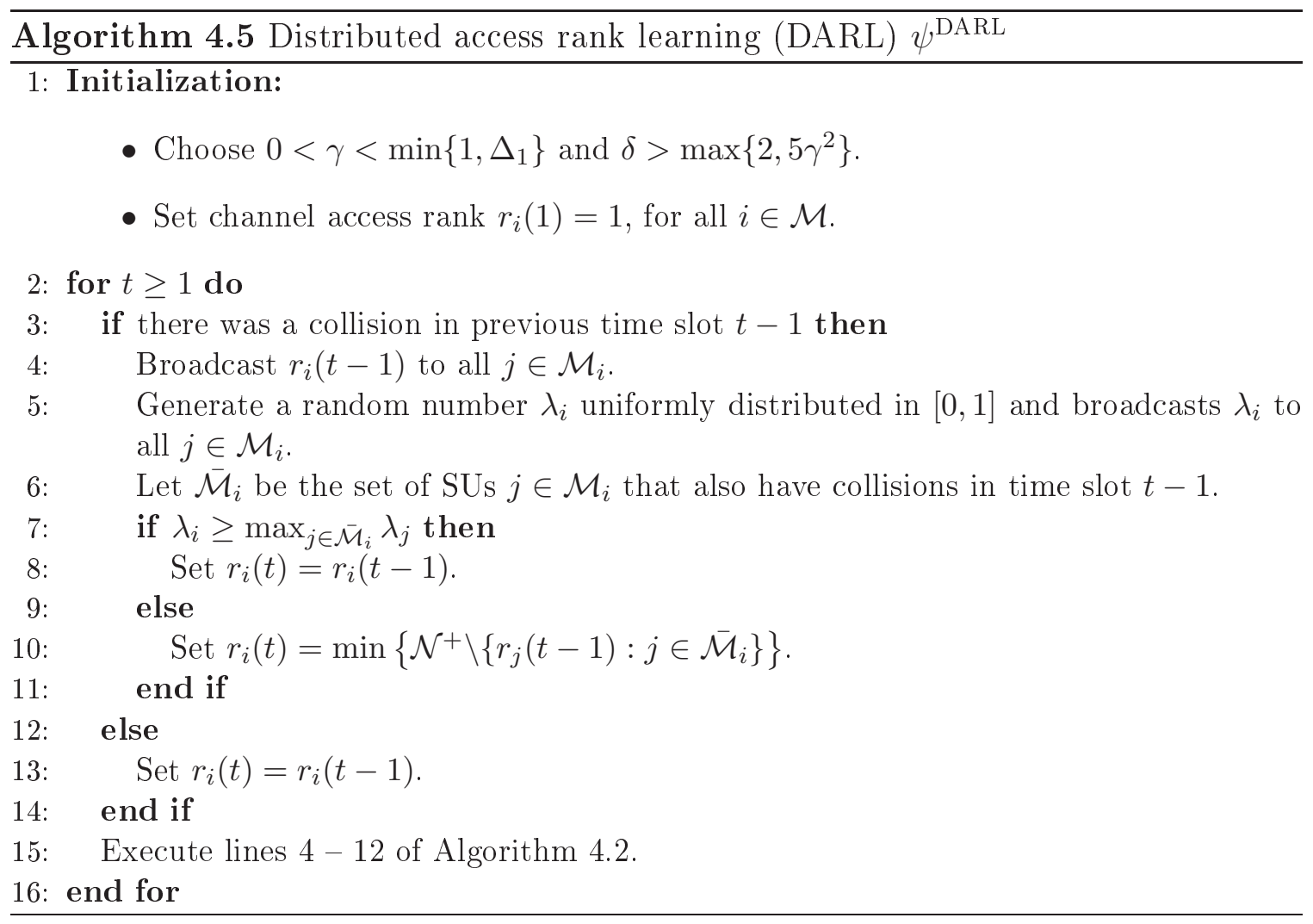

Through this process, with an increasing likelihood over time, each SU is allocated the minimal available channel access rank that is different from its neighbors and the channel statistics are learned at the same time. The DARL policy does not require two separate initial stages to learn the access ranks. However, since the access ranks are assigned somewhat randomly and do not take into consideration the number of SUs with the same access rank, it is likely to have a higher regret compared to the CARL policy.

\subsubsection{Regret Bounds}

In this section, we derive theoretical bounds on the regret (4.2) achieved by our proposed CARL and DARL policies. As benchmark comparisons, we also derive 
bounds under our spatial spectrum reuse framework for the random access policy [30], the TDFS policy [31] and the adaptive randomization policy [30], which we denote by $\psi^{\text {rand }}, \psi^{\text {TDFS }}$ and $\psi^{\text {adapt }}$, respectively. We first describe these policies briefly below.

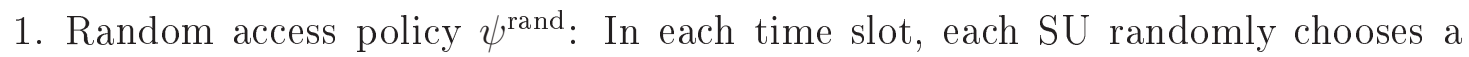
channel $j \in \mathcal{N}$ to sense. The SU transmits if the channel is found to be idle.

2. TDFS policy $\psi^{\mathrm{TDFS}}$ : As describing the policy accurately requires some technical details, we refer the reader to [31]. We summarize the policy here briefly. Without going into the technical details, the TDFS policy is intuitively similar to a policy in which each SU accesses the $M$ best channels in a round-robin fashion. A different offset, based on each SU's identity, in the channel access sequence is used to ensure that every $\mathrm{SU}$ is assigned a different channel access rank in each time slot. Each SU determines the $M$ best channels by running parallel Lai-Robbins single-player policies [93]. To determine the $k$-th best channel, a SU removes the $k-1$ channels it considers to be the best (these are channels it has attempted to access in the previous $k-1$ time slots). It then considers the subsequence time slots that also have the same set of $k-1$ best channels removed, and performs a Lai-Robbins single-player policy on these time slots, using only the remaining $N-k+1$ channels to find the best channel amongst these. For the purpose of our discussion, the main point to note is that each $\mathrm{SU}$ is assigned the channel access ranks $1, \ldots, M$ in a round-robin fashion over time slots. This ensures "fairness" in accessing the best channel, which we do not address in this chapter. 
3. Adaptive randomization policy $\psi^{\text {adapt }}$ Every SU is initially assigned the channel access rank of 1 . Then, in each time slot, each $\mathrm{SU} i$ randomizes its channel access rank only if there is a collision in the previous slot, otherwise it retains its channel access rank from the previous time slot. Suppose the channel access rank is $r$. The $r$-th highest channel based on SU $i$ 's sample-mean statistics [29] given by

$$
\bar{X}_{i, j}(n)+\sqrt{\frac{2 \log n}{T_{i, j}(n)}},
$$

for each channel $j \in \mathcal{N}$, is chosen to be sensed. The SU transmits if the channel is found to be idle.

It is clear that since there is a positive probability that the optimal channel access ranks are not found in $\psi^{\mathrm{CARL}}$ and $\psi^{\mathrm{DARL}}$, these policies have $\Theta(n)$ regret in general. The same can be said of our benchmark policies as shown below.

Proposition 4.2. Under spatial spectrum reuse, if the graph $G$ is incomplete and has a connected component of size at least two, then $\psi^{\text {rand }}, \psi^{\text {TDFS }}$, and $\psi^{\text {adapt }}$ each has $\Theta(n)$ regret.

Proof. For any policy $\psi$, we have

$$
\begin{aligned}
R(n, \psi) & =n \sum_{i \in \mathcal{M}} \mu_{\pi^{*}(i)}-\sum_{i \in \mathcal{M}} \sum_{j \in \mathcal{N}^{+}} \mu_{j} \mathbb{E}\left[V_{i, j}(n)\right] \\
& \geq \sum_{i \in \mathcal{M}} \sum_{j>\pi^{*}(i)}\left(\mu_{\pi^{*}(i)}-\mu_{j}\right) \mathbb{E}\left[T_{i, j}(n)\right] .
\end{aligned}
$$


In the policy $\psi^{\text {rand }}$, each SU randomly chooses a channel to sense in each time slot. Consider a SU $i^{\prime}$ with $\pi^{*}\left(i^{\prime}\right)=1$. From (4.10), we have

$$
\begin{aligned}
R\left(n, \psi^{\text {rand }}\right) & \geq\left(\mu_{1}-\mu_{2}\right) \sum_{j=2}^{N} \mathbb{E}\left[T_{i^{\prime}, j}(n)\right] \\
& =\left(\mu_{1}-\mu_{2}\right) \frac{N-1}{N} n .
\end{aligned}
$$

Therefore, $\psi^{\text {rand }}$ has $\Theta(n)$ regret.

We next consider the policy $\psi^{\text {TDFS }}$. Every SU uses the Lai-Robbins single-player policy to determine the best channel. For all time slots $n$ sufficiently large, the probability that all SUs identify channel 1 as the best channel is bounded away from zero. From the proposition assumptions, there is a $\mathrm{SU} i$ in $G$ with degree less than $M-1$. Since the TDFS policy assigns to each SU channel access ranks in a round-robin fashion over time slots, there exists at least one time slot out of every $M$ slots such that all SUs in $i \cup \mathcal{M}_{i}$ do not have channel access rank 1 . Consider a policy $\psi^{\prime}$ that is the same as $\psi^{\text {TDFS }}$ but which assigns channel access rank 1 to $\mathrm{SU} i$ in each of these time slots starting from some time $n$ sufficiently large. Then, $R\left(n, \psi^{\mathrm{TDFS}}\right) / n \geq R\left(n, \psi^{\prime}\right) / n+c\left(\mu_{1}-\mu_{2}\right) / M$ for some positive constant $c>0$. This implies that $\psi^{\text {TDFS }}$ has $\Theta(n)$ regret.

In the policy $\psi^{\text {adapt}}$, each SU randomizes the channel selection from $\mathcal{N}^{+}$only if there is a collision in the previous time slot. Since the graph $G$ has a connected component of size at least two, not all feasible solutions in (P0) are optimal. There is a positive probability that at the second iteration of $\psi^{\text {adapt }}$, the SUs are assigned 
channel access ranks corresponding to a feasible but suboptimal solution of (P0). Subsequently, the channel access ranks do not change. Therefore, $\psi^{\text {adapt }}$ has $\Theta(n)$ regret. The proof of the proposition is now complete.

Although $\psi^{\mathrm{CARL}}$ has $\Theta(n)$ regret, as shown in the following proposition, it is able to achieve $\Theta(\log n)$ regret if its distributed graph coloring step in Algorithm 4.3 produces the optimal graph coloring. The proof of Proposition 4.3 is similar to that for Theorem 4.1 and is omitted here. As explained in Section 4.3, Algorithm 4.3 is likely to produce an optimal graph coloring for a sparse graph $G$. This explains our observation in our simulation results that $\psi^{\mathrm{CARL}}$ has better regret performance than the benchmark policies although all of them have linear regret.

Proposition 4.3. Suppose that the output from Algorithm 4.4 satisfy $r_{i}=\pi^{*}(i)$ for all $i \in \mathcal{M}$, then the policy $\psi^{\mathrm{CARL}}$ has $\Theta(\log n)$ regret.

\subsection{Simulation Results}

In this section, we verify the performance of our proposed policies by simulations. We first consider small size simple graphs, and then provide simulation results on large size random graphs.

\subsubsection{Small Size Graphs}

Suppose that there are $M=9$ SUs and $N=9$ orthogonal PU channels. The idle probabilities of the PU channels are evenly spaced values from 0.1 to 0.9 . We apply 
our proposed policy in three connected interference graphs, which are the nine-node ring graph, a grid graph and a randomly generated graph (See Figure 4-3). Similar types of graphs have been adopted to evaluate spatial channel reuse performance in [60].

In Algorithms 4.2, 4.3 and 4.4, we set $T_{1}=9, T_{2}=300, \delta=5.1$ and $\gamma=0.1$. For $\psi^{\mathrm{CCA}}$, we let $l_{0}=2$ and $l_{k}=2 l_{k-1}$ for $k \geq 1$.

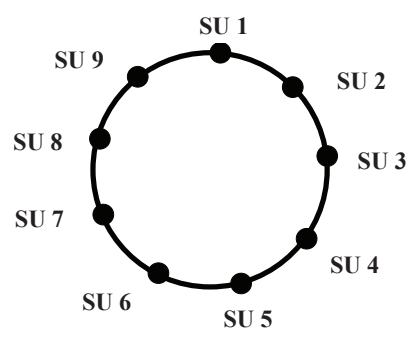

(a)

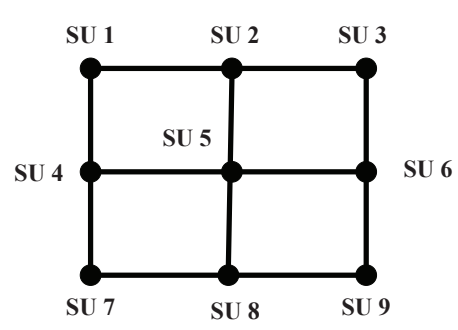

(b)

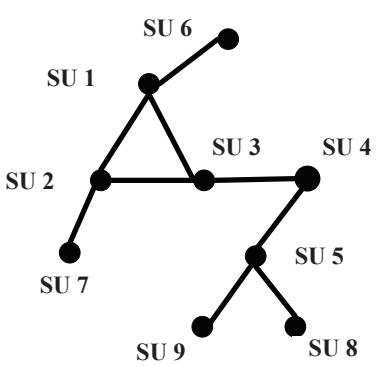

(c)

Figure 4-3: Interference graphs of three CRNs (a) a ring graph (b) a grid graph (c) a randomly generated graph.

In Figures 4-4, 4-5 and 4-6, we observe that $\psi^{\mathrm{CCA}}, \psi^{\mathrm{CARL}}$ and $\psi^{\mathrm{DARL}}$ outperform the policies $\psi^{\text {rand }}, \psi^{\text {TDFS }}$ and $\psi^{\text {adapt }}$ in all three interference graphs, where the regrets of $\psi^{\mathrm{CCA}}$ and $\psi^{\mathrm{CARL}}$ are approximately a constant multiple of $\log n$. Moreover, the regret of $\psi^{\mathrm{CARL}}$ is close to that of $\psi^{\mathrm{CCA}}$ because Algorithm 4.4 manages to find optimal or near-optimal channel access ranks for all SUs in these graphs. We also note that $\psi^{\text {DARL }}$ has worse regret than $\psi^{\mathrm{CARL}}$, but still performs better than the benchmark algorithms. 


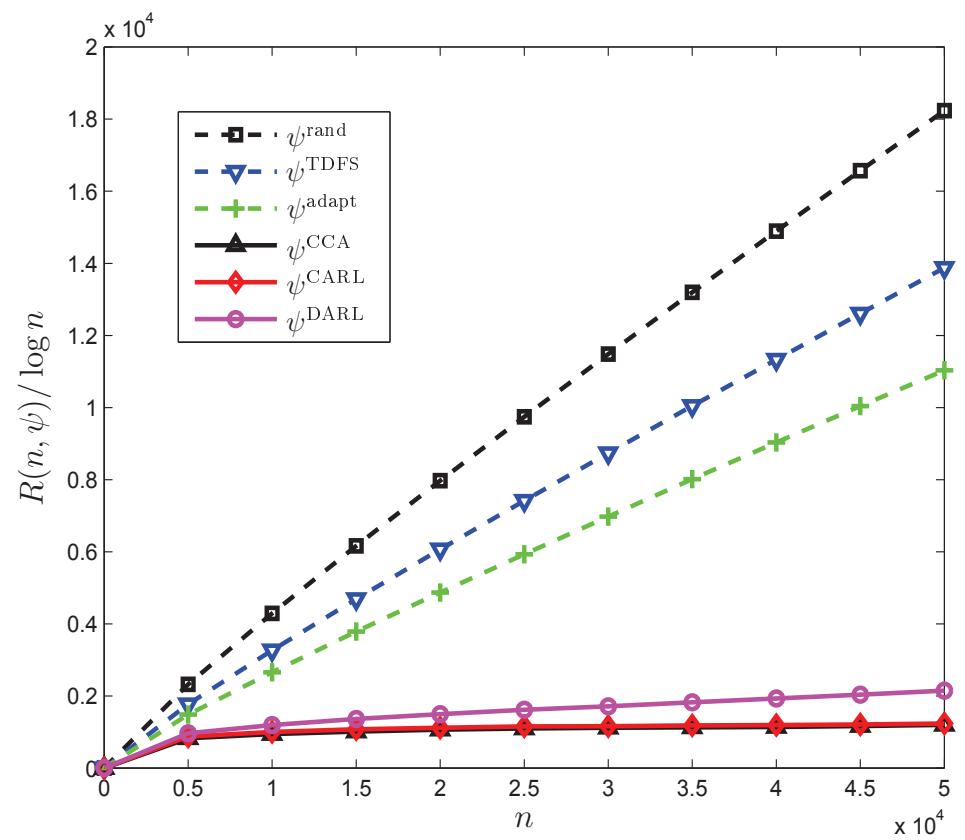

Figure 4-4: Normalized regret $\frac{R(n, \psi)}{\log n}$ vs. time slot $n$ on ring graph.

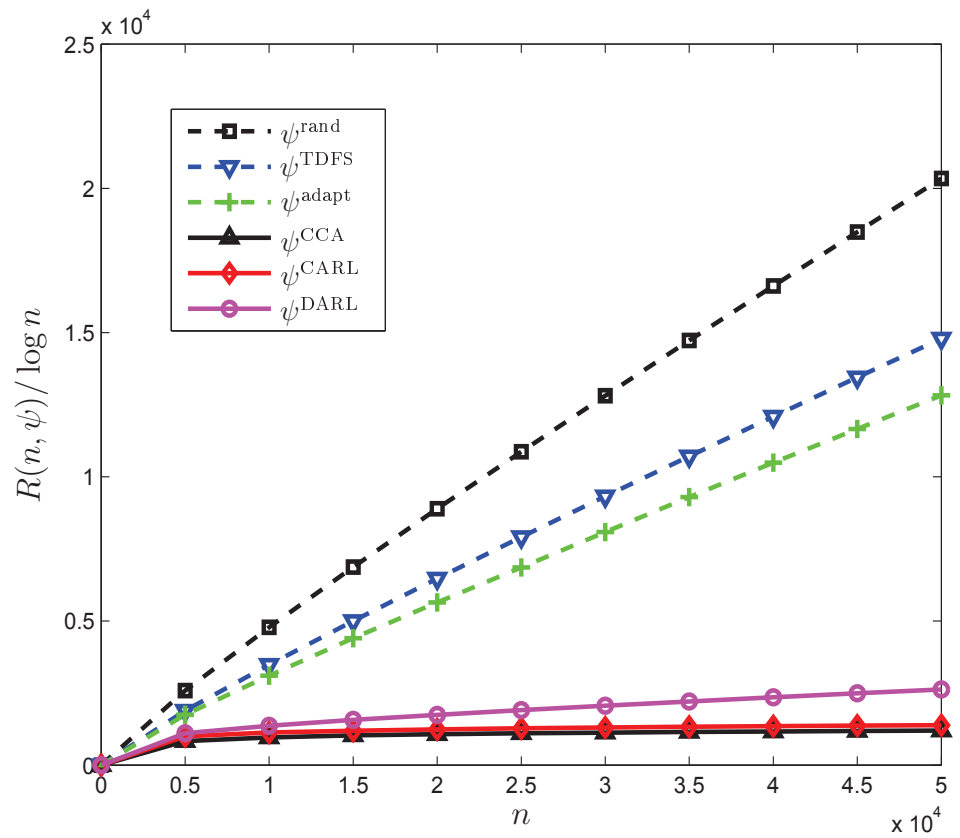

Figure 4-5: Normalized regret $\frac{R(n, \psi)}{\log n}$ vs. time slot $n$ on grid graph. 


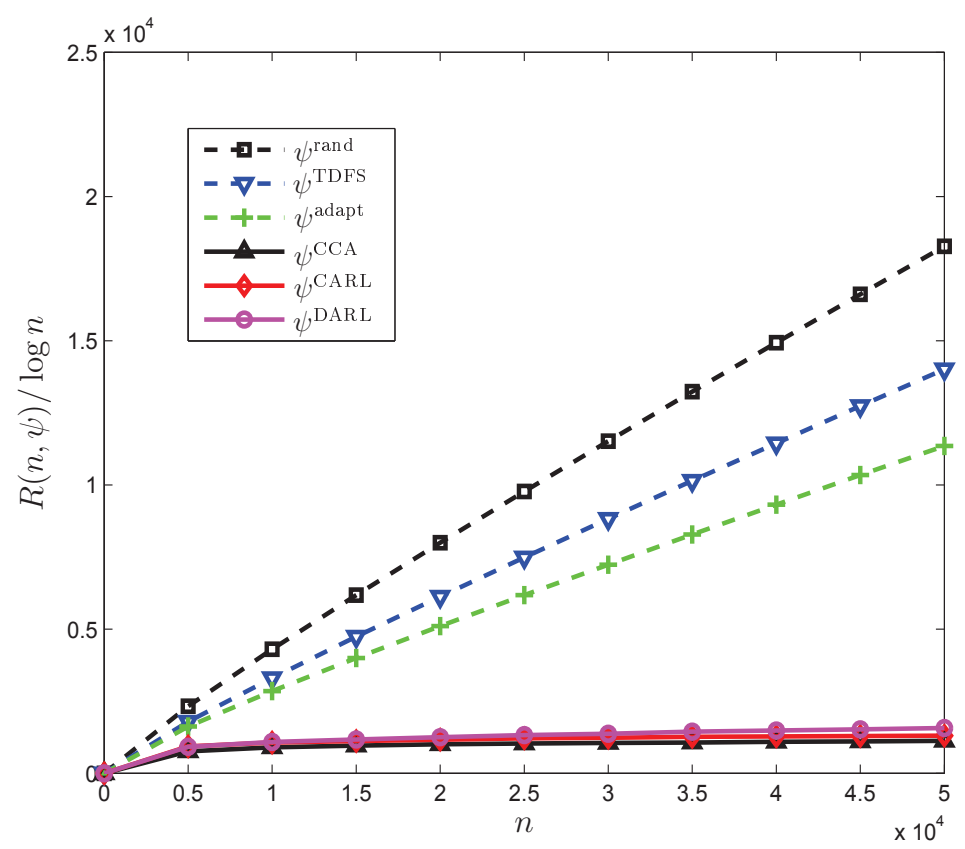

Figure 4-6: Normalized regret $\frac{R(n, \psi)}{\log n}$ vs. time slot $n$ on randomly generated graph.

\subsubsection{Large Size Random Graphs}

In this subsection, we consider large size random graphs that have $M=100 \mathrm{SUs}$ and $N=100$ orthogonal PU channels. The idle probabilities of the PU channels are $[0.9,0.8,0.7,0.6,0.5,0.495,0.490, \ldots, 0.025]$. In Algorithms 4.2, 4.3 and 4.4, we set $T_{1}=100, T_{2}=3500, \delta=5.1$ and $\gamma=0.1$. We evaluate the performance of our proposed policies and that of the benchmark policies on the following:

(i) Erdös-Rényi (ER) graphs: 500 instances of Erdos-Renyi random graphs with $M$ nodes and different probabilities of attachment [138]

(ii) Random connection (RC) graphs: 500 random graphs with $M$ nodes and different number of edges. Edges are generated sequentially, and each edge is formed by choosing two distinct nodes uniformly at random and connecting them if 
they are not already connected.

Table 4.2 shows the percentage of simulation runs in which the policy $\psi^{\mathrm{CARL}}$ and the policy $\psi^{\text {DARL }}$ found the correct graph chromatic number $\chi(G)$. We can see that $\psi^{\mathrm{CARL}}$ and $\psi^{\mathrm{DARL}}$ estimate the chromatic number $\chi(G)$ better when the network is sparse. For other policies $\psi^{\text {rand }}, \psi^{\text {TDFS }}$ and $\psi^{\text {adapt }}$, simulation results show that they are not able to estimate the correct graph chromatic number.

Table 4.2: Performance of $\psi^{\mathrm{CARL}}$ and $\psi^{\mathrm{DARL}}$ in finding the correct chromatic number.

\begin{tabular}{|c|c|c|c|}
\hline ER attachment probability & 0.05 & 0.1 & 0.2 \\
\hline $\begin{array}{l}\psi^{\text {CARL-Percentage instances }} \\
\text { where correct } \chi(G) \text { is found }\end{array}$ & $71.1 \%$ & $53.7 \%$ & $34 \%$ \\
\hline $\begin{array}{l}\psi^{\text {DARL }} \text {-Percentage instances } \\
\text { where correct } \chi(G) \text { is found }\end{array}$ & $64.8 \%$ & $56.67 \%$ & $23.3 \%$ \\
\hline $\mathrm{RC}$ number of edges & 200 & 500 & 1000 \\
\hline $\begin{array}{l}\psi^{\text {CARL }} \text {-Percentage instances } \\
\text { where correct } \chi(G) \text { is found }\end{array}$ & $60.4 \%$ & $51 \%$ & $36 \%$ \\
\hline $\begin{array}{l}\psi^{\text {DARL-Percentage }} \text { instances } \\
\text { where correct } \chi(G) \text { is found }\end{array}$ & $50.1 \%$ & $38.3 \%$ & $26 \%$ \\
\hline
\end{tabular}

For the instances in which $\psi^{\mathrm{CARL}}$ and $\psi^{\mathrm{DARL}}$ estimated the correct chromatic number, let

$$
D=\frac{1}{M} \sum_{i=1}^{M}\left|r_{i}-\pi^{*}(i)\right|
$$

be the average error in finding the correct channel access ranks. Table 4.3 shows that the policy $\psi^{\mathrm{CARL}}$ and the policy $\psi^{\mathrm{DARL}}$ could allocate channel access ranks for sparse random graphs with a relatively small error. Moreover, we also find that the policy $\psi^{\text {DARL }}$ has a larger error than the policy $\psi^{\mathrm{CARL}}$ in finding the correct channel access 
ranks.

Table 4.3: Performance of $\psi^{\mathrm{CARL}}$ and $\psi^{\mathrm{DARL}}$ in finding the correct channel access ranks.

\begin{tabular}{|l|l|l|l|}
\hline ER attachment probability & 0.05 & 0.1 & 0.2 \\
\hline$\psi^{\text {CARL }}-D$ & $\mathbf{0 . 2 6 8}$ & 0.379 & 0.664 \\
\hline \hline$\psi^{\text {DARL }}-D$ & $\mathbf{0 . 9 3 3}$ & 1.502 & 2.5 \\
\hline \hline RC number of edges & 200 & 500 & 1000 \\
\hline$\psi^{\text {CARL }}-D$ & $\mathbf{0 . 2 1 9}$ & 0.398 & 0.658 \\
\hline$\psi^{\text {DARL }}-D$ & $\mathbf{0 . 7 1 8}$ & 1.493 & 2.58 \\
\hline
\end{tabular}

Finally, we show the regrets in Figure 4-7 and Figure 4-8 when the graph is a randomly generated ER graph with attachment probability 0.05 and a RC graph with 200 edges. We compare the average regrets using 500 trials for all the policies. We observe that the regret of $\psi^{\mathrm{CCA}}$ is again approximately a constant multiple of $\log n$ and regrets using other policies on both types of random graphs increase linearly over time. However, our policies $\psi^{\mathrm{CARL}}$ and $\psi^{\mathrm{DARL}}$ give a much smaller regret than the benchmark policies.

\subsection{Summary}

In this chapter, we have investigated temporal-spatial spectrum reuse in cognitive radio networks using a multi-user $\mathrm{MAB}$ approach. We have proposed a centralized channel allocation policy for finding an optimal channel allocation and learning the statistics of the channels. We showed that this policy is order-optimal with logarithmic regret, but requires solving a NP-complete optimization problem at exponentially increasing time intervals. To avoid that and to overcome the requirement of central- 


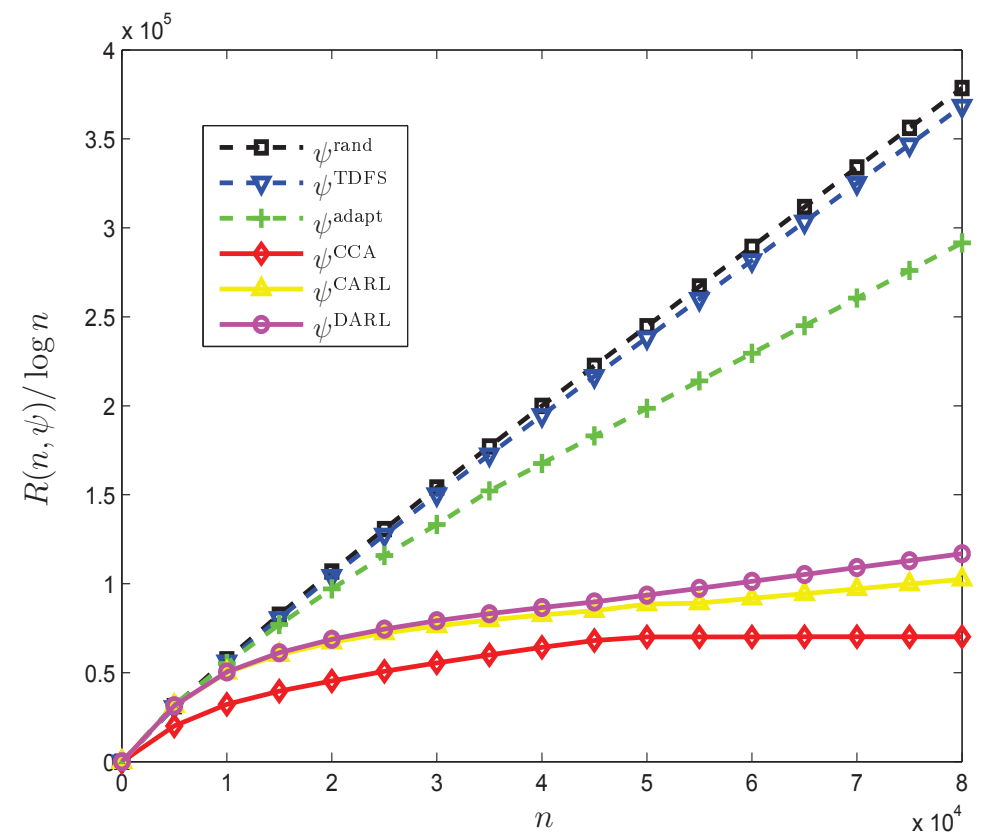

Figure 4-7: Normalized regret $\frac{R(n, \psi)}{\log n}$ vs. time slot $n$ on ER graphs.

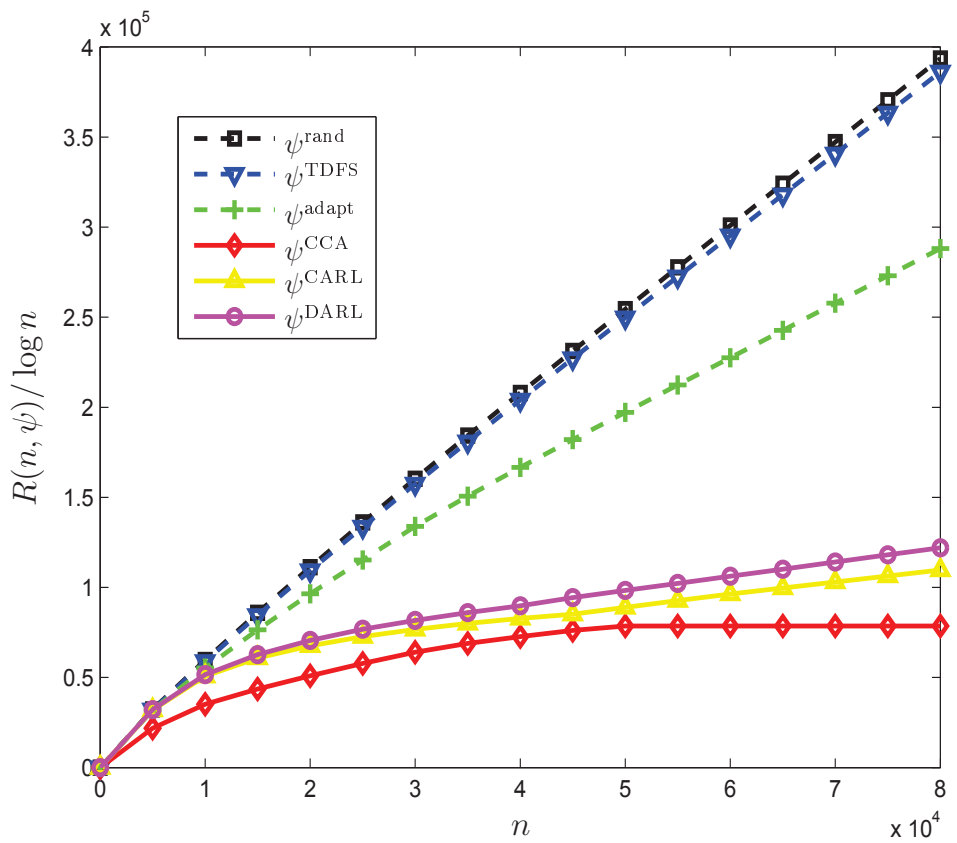

Figure 4-8: Normalized regret $\frac{R(n, \psi)}{\log n}$ vs. time slot $n$ on RC graphs. 
ized processing, we also proposed two heuristic distributed policies, which however have linear regrets. The first distributed policy utilizes a distributed greedy graph coloring method and a distributed average consensus method in the learning process to find the optimal channel access rank for each SU. This policy requires synchronization amongst the SUs. In the second distributed policy, we let each SU determine their channel access ranks locally, which removes the requirement for synchronization. Simulation results suggested that our proposed policies outperform current policies in the literature, which do not consider spatial channel reuse. 
THIS PAGE INTENTIONALLY LEFT BLANK 


\section{Chapter 5}

\section{Collaborative Learning For}

\section{Temporal-Spatial Spectrum Reuse}

In Chapter 4, we have proposed three learning policies for temporal-spatial OSA, in which SUs could learn their channel access ranks and independently learn the statistics of channels without information exchange among themselves. In a large-scale network with many SUs, if the required regret caused by exploration of non-optimal channels for a $\mathrm{SU}$ is $\delta$, then the total exploration regret introduced by all SUs would be $M \delta$, which will be extremely huge due to a large $M$ and the lack of communication. This motivates us to study a large-scale MAB problem in a distributed environment where learning is performed by independent SUs that may take actions, obtain sensing observations and exchange information with neighboring SUs. Intuitively, with some information exchange, SUs will operate more wisely and learn the ranks of the channels faster.

In this chapter, we firstly describe the collaborative MAB problem in Section 
5.1. Then, we propose three collaborative learning polices respectively using channel sensing observations, empirical estimates of channel idle probabilities and estimated channel ranks of SUs in Section 5.2. In Section 5.3, we provide simulation results which can verify the performance of our proposed collaborative learning policies.

\subsection{Problem Formulation}

We consider a collaborative MAB problem for temporal-spatial spectrum reuse in which SUs collaboratively learn the statistics of channels through exchanging some information with neighboring SUs at the end of each time slot. We adopt the same multi-user multi-armed bandit problem formulation, notations and definitions introduced in Section 4.1 of Chapter 4.

The proposed three collaborative learning policies are based on the distributed policy $\psi^{\text {CARL }}$ described in Section 4.3 of Chapter 4 . The first two stages of the policy $\psi^{\mathrm{CARL}}$ will be adopted by our proposed collaborative learning polices. We assume that some pre-located channel access ranks are already known by SUs after applying the distributed graph coloring algorithm mentioned in Algorithm 4.3 and the distributed channel access rank determination algorithm described in Algorithm 4.4. All the proposed collaborative learning policies perform the following procedures by using different collaborative channel learning algorithms as the last step, which will be introduced respectively in Algorithm 5.1, Algorithm 5.2 or Algorithm 5.3:

1. distributed graph coloring algorithm (Algorithm 4.3);

2. distributed channel access rank determination method (Algorithm 4.4); and 
3. collaborative channel learning: Collaborative Average Observation Channel Learning (CAOCL) policy in Algorithm 5.1, Collaborative Average Mean Channel Learning (CAMCL) policy in Algorithm 5.2 or Collaborative Channel Rank Learning (CCRL) policy in Algorithm 5.3.

In this chapter, we assume that there is no restriction on the size of each message and there is enough bandwidth of the underlying communication network so that each SU could share some information in each round.

\subsection{Collaborative Channel Learning}

In this section, we propose three collaborative channel learning policies by adopting the idea of collaborative learning. We describe these policies respectively in the following sections. We use the same notations which have been described in Chapter 4.

\subsubsection{CAOCL Policy}

At the end of each time slot $n$, after exchanging sensing observations with neighboring SUs, each SU $i$ performs an average of the sensing observations of its neighboring SUs for their selected channels $\rho_{j}(n)$, for $j \in \mathcal{M}_{i}$, and updates $T_{i, j}(n)$ and $\bar{X}_{i, j}(n)$. Let $r_{i}$ be the random access rank for $\mathrm{SU} i$, which is obtained from the second step in Algorithm 4.4. Let $\left|\mathcal{M}_{i}\right|$ denote the number of neighboring SUs for SU $i$. At the time slot $n+1$, with probability $1-\epsilon_{n+1}$, SU $i$ selects a channel with the $r_{i}$-th highest empirical idle probability estimate based on the updated $T_{i, j}(n)$ and $\bar{X}_{i, j}(n)$, 
otherwise it uniformly chooses a random channel from the channel set $\mathcal{N}$. By using this policy, we can easily observe that if $k \in\left|\mathcal{M}_{i}\right|$ neighboring SUs of SU $i$ happen to select to sense channel $j$ at the time slot $n$, it is equivalent as $\mathrm{SU} i$ itself explores $k$ times of channel $j$ at one moment. Then, its channel exploration time will be largely shortened. We call this the Collaborative Average Observation Channel Learning (CAOCL) policy denoted by $\psi^{\mathrm{CAOCL}}$. The detail of the learning process is described in Algorithm 5.1.

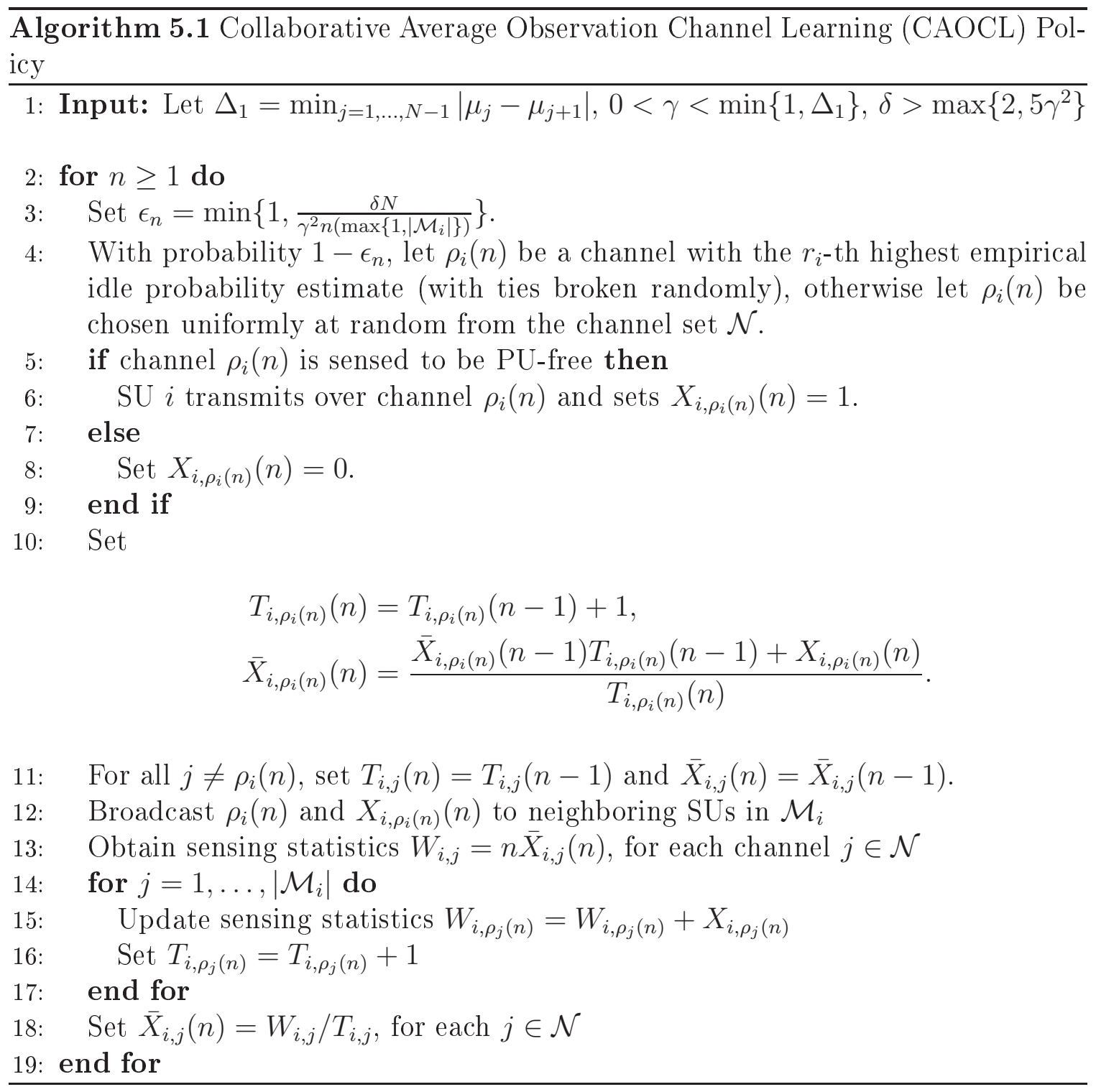


To illustrate the averaging approach in Algorithm 5.1, we give an example of channel statistic calculation in Table 5.1 using an interference graph of a CRN with three SUs in Figure 5-1. An edge between two SUs indicates that they interfere with each other when utilizing the same channel. We assume that at the time slot $n$, each SU already chose to sense a channel instance $\rho_{i}(n)$. We show the results of statistic calculation before and after information exchange with neighboring SUs at the end of time slot $n$.

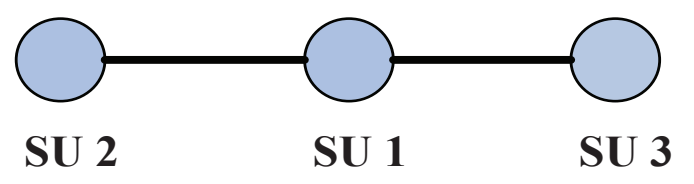

Figure 5-1: An interference graph of a CRN with three SUs

In Table 5.1, we assume that the selected channels for SU 1, SU 2 and SU 3 are channel 1, channel 2 and channel 2 respectively at the time slot $n$. After information exchange with neighboring SU 2 and SU 3, SU 1 will update the empirical idle probability estimate of channel 2 using the collected sensing observations from its neighbors SU 2 and SU 3. In the meantime, SU 2 and SU 3 will individually update their idle probability estimate of channel 1 based on the received sensing observation from their neighbor SU 1.

\subsubsection{CAMCL Policy}

For privacy and security considerations, it is not practical to demand all SUs to broadcast their sensing observations to their neighbors at each time slot. Therefore, at the end of each time slot $n$, instead of directly averaging the sensing observations 
Table 5.1: Statistic calculation in Algorithm 5.1

\begin{tabular}{|c|l|l|l|}
\hline SU $i$ & Information for Exchange & $\begin{array}{l}\text { Channel Selection Instance } \\
\rho_{i}(n) \text { in Time Slot } n\end{array}$ & Statistic Calculation \\
\hline 1 & $\rho_{1}(n)$ and $X_{1, \rho_{1}(n)}(n)$ & $\rho_{1}(n)=1$ & $\begin{array}{l}\bar{X}_{1,2}(n) \\
=\frac{n \bar{X}_{1,2}(n)+X_{2,2}(n)+X_{3,2}(n)}{T_{1,2}+2}\end{array}$ \\
\hline 2 & $\rho_{2}(n)$ and $X_{2, \rho_{2}(n)}(n)$ & $\rho_{2}(n)=2$ & $\begin{array}{l}\bar{X}_{2,1}(n) \\
=\frac{n \bar{X}_{2,1}(n)+X_{1,1}(n)}{T_{2,1}+1}\end{array}$ \\
\hline 3 & $\rho_{3}(n)$ and $X_{3, \rho_{3}(n)}(n)$ & $\rho_{3}(n)=2$ & $\begin{array}{l}\bar{X}_{3,1}(n) \\
=\frac{n \bar{X}_{3,1}(n)+X_{1,1}(n)}{T_{3,1}+1}\end{array}$ \\
\hline
\end{tabular}

of neighboring SUs on their selected channels, we design a collaborative learning policy that enables each SU to perform information weighted average consensus on the empirical idle probability estimates $\bar{X}_{i, \rho_{j}(n)}(n)$ received from its neighboring SUs, $\forall j \in \mathcal{M}$ on their selected channels $\rho_{j}(n)$. We call this the Collaborative Average Mean Channel Learning (CAMCL) policy denoted by $\psi^{\mathrm{CAMCL}}$, which is introduced in Algorithm 5.2. Let $\alpha_{i, j}$ denote the averaging weight used by each $\mathrm{SU} i$ for combining the estimation from its neighboring $\mathrm{SU} j$, for each $j \in \mathcal{M}_{i}$. The averaging weight for its own estimate is denoted by $\alpha_{i, i}$.

In Table 5.2, we also provide an example to describe the channel statistic calculation process in Algorithm 5.2. We assume that at the time slot $n$, the channel selections of SU 1, SU 2 and SU 3 are still the same as the settings in Table 5.1, which are channel 1, channel 2 and channel 2. Instead of using the sensing observations of its neighboring SUs, each SU applies an average consensus algorithm [28] on their empirical idle probability estimates. In this example, we adopt the uniform weight consensus algorithm [28] and simply assume that the consensus weights $\alpha_{i, i}$ and $\alpha_{i, i}$ satisfy $\alpha_{i, i}=\alpha_{i, j}=\frac{1}{\left|\mathcal{M}_{i}\right|+1}$ for each SU $i$ and each of its neighboring SUs $j \in \mathcal{M}_{i}$. 


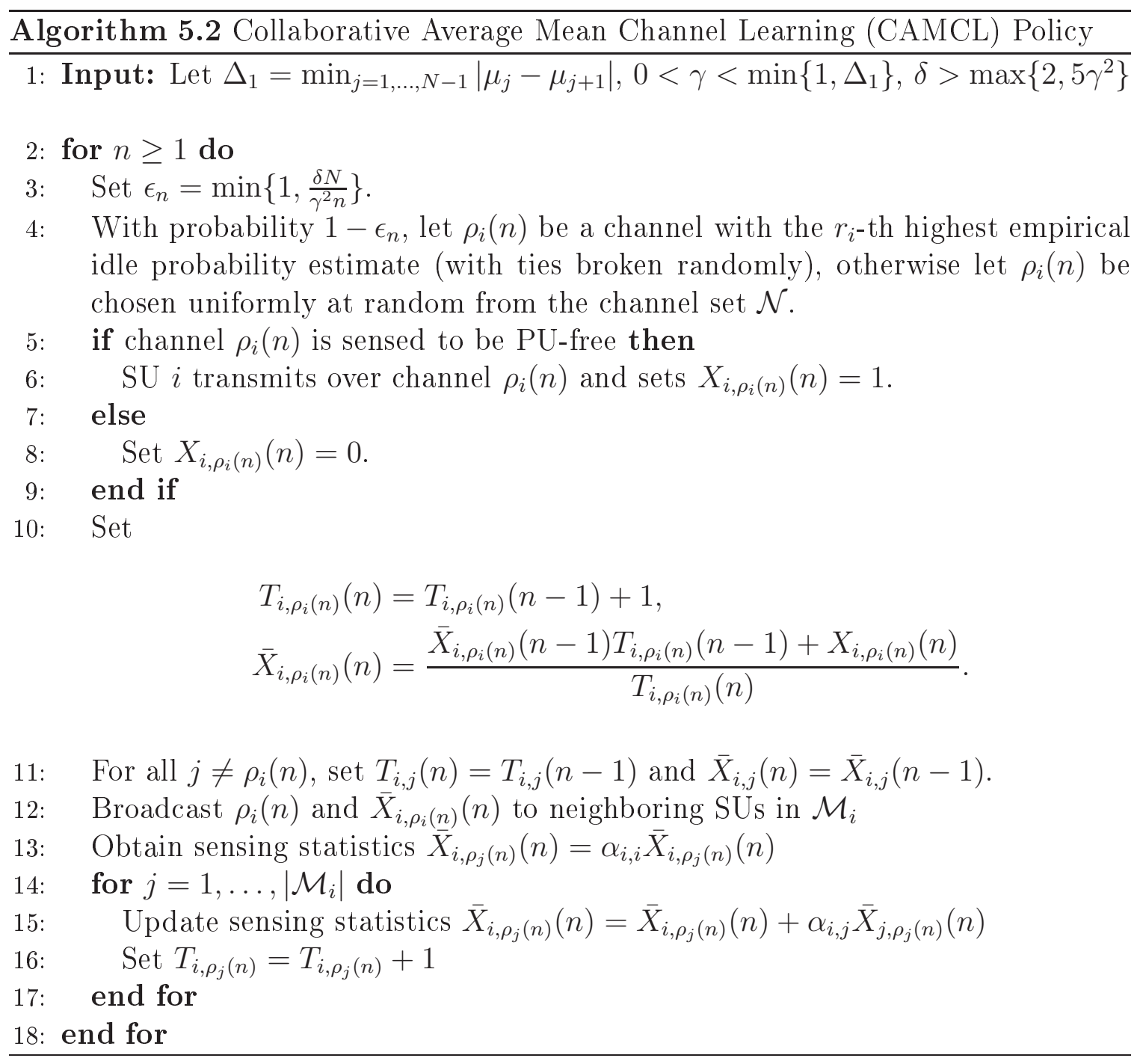

Table 5.2: Statistic calculation in Algorithm 5.2

\begin{tabular}{|c|l|l|l|}
\hline SU $i$ & Information for Exchange & $\begin{array}{l}\text { Channel Selection Instance } \\
\rho_{i}(n) \text { in Time Slot } n\end{array}$ & Statistic Calculation \\
\hline 1 & $\rho_{1}(n)$ and $\bar{X}_{i, \rho_{1}(n)}(n)$ & $\rho_{1}(n)=1$ & $\begin{array}{l}\bar{X}_{1,2}(n)=\frac{1}{3} \bar{X}_{1,2}(n) \\
+\sum_{j \in \mathcal{M}_{1}} \frac{1}{3} \bar{X}_{j, 2}(n)\end{array}$ \\
\hline 2 & $\rho_{2}(n)$ and $\bar{X}_{i, \rho_{2}(n)}(n)$ & $\rho_{2}(n)=2$ & $\begin{array}{l}\bar{X}_{2,1}(n)=\frac{1}{2} \bar{X}_{2,1}(n) \\
+\frac{1}{2} \bar{X}_{1,1}(n)\end{array}$ \\
\hline 3 & $\rho_{3}(n)$ and $\bar{X}_{i, \rho_{3}(n)}(n)$ & $\rho_{3}(n)=2$ & $\begin{array}{l}\bar{X}_{3,1}(n)=\frac{1}{2} \bar{X}_{3,1}(n) \\
+\frac{1}{2} \bar{X}_{1,1}(n)\end{array}$ \\
\hline
\end{tabular}




\subsubsection{CCRL Policy}

In many real applications, the sensing observations and the empirical idle probability estimates of neighboring SUs are usually not available due to a stricter privacy requirement. Therefore, it is interesting to investigate whether we could design a collaborative learning policy without using these estimated channel statistics. We propose a collaborative learning policy that uses the estimated channel ranks collected from neighboring SUs and then performs a majority voting to obtain a good ranking result for channels.

At the end of each time slot $n$, let $L_{n}(i,:)$ denote a vector for each SU $i$ to record the channel identities with ranks $1,2, \ldots, N$ after sorting their empirical idle probability estimates $\bar{X}_{i, j}(n), \forall j \in \mathcal{N}$ in decreasing order. The channel with the largest empirical idle probability will be ranked first. Let $A \backslash B$ denote the set difference between set $A$ and set $B$, which is defined by $A \backslash B=\{x: x \in A$ and $x \notin B\}$. We assume that the initial ranks for all channels satisfy $L_{1}(i,:)=\{1, \cdots, N\}$ for each $\mathrm{SU} i$. Let $U_{i}=\left\{i \cup j, \forall j \in \mathcal{M}_{i}\right\}$ be the voting group for SU $i$ including itself and all its neighboring SUs, in which all members will join the voting process. Each $\mathrm{SU} i$ then adopts the idea of majority voting and re-evaluates the channel ranks $L_{n}(i,:)$ by reaching a consensus on the received channel ranks which are collected from the voting group $U_{i}$. Let $Q_{i}$ be a $\left(\left|\mathcal{M}_{i}\right|+1\right) \times N$ matrix for each SU $i$ to store the ranks $L_{n}(k,:)$, where $k \in U_{i}$. We set $Q_{i}(1,:)=L_{n}(i,:)$ and $Q_{i}\left(2:\left|\mathcal{M}_{i}\right|+1,:\right)=L_{n}(j,:)$, for all $j \in \mathcal{M}_{i}$. We follow a simple majority voting rule, i.e., if more than half of SUs in the voting group $U_{i}$ agree channel $j$ as "good" with the rank $r$ at the time slot $n, \mathrm{SU}$ 
$i$ will set $L_{n+1}(i, r)=j$. Otherwise, it will keep using the same rank for channel $j$. In some cases, there may be two channels $j, k$ voted by SUs in the voting group $U_{i}$ with the same rank $w$. If we assume that the identities of these two channels satisfy $j<k$, SU $i$ will allocate a lower rank to the channel with a smaller channel identity by updating $L_{n+1}(i, w)=j$ and $L_{n+1}(i, w+1)=k$. And it will keep the same order of ranks for the other channels so that for any two channels with the rank $a, b$ and $a<b$ in the vector $L_{n}(i,:)$, they will be allocated to the ranks with the same order $a^{\prime}$, $b^{\prime}$ satisfying $a^{\prime}<b^{\prime}$ in the vector $L_{n+1}(i,:)$, which will be used in time slot $n+1$. We call this the Collaborative Channel Rank Learning (CCRL) policy and it is denoted by $\psi^{\mathrm{CCRL}}$, which is described in Algorithm 5.3.

Table 5.3 gives an example to illustrate the channel re-ranking step for a SU $i$ after information exchange with its neighboring SUs. We assume that there are four channels in the CRN. At the time slot $n$, we assume that SU $i$ already has a vector $L_{n}(i,:)$ for storing the channel identities with ranks $1,2, \ldots, N$ after ranking their empirical idle probability estimates $\bar{X}_{i, j}(n), \forall j \in \mathcal{N}$ in decreasing order. For SU $i$, let channel identities with the ranks from 1 to 4 be channel 1, channel 3, channel 4 and channel 2. And channel 1 is identified to be the "best" channel by SU $i$. Suppose the voting group of SU $i$ has agreed that both channel 3 and channel 4 should be ranked third. Then, according to our proposed algorithm 5.3, we should give a lower rank to the channel with a smaller channel identity. Therefore, we set $L_{n+1}(i, 3)=3$ and $L_{n+1}(i, 4)=4$. For the channel 1 and channel 2, they will be given the ranks with the same order as that in $L_{n}(i,:)$, in which channel 1 still has a smaller rank than channel 2. We can observe that this policy could effectively protect the privacy 


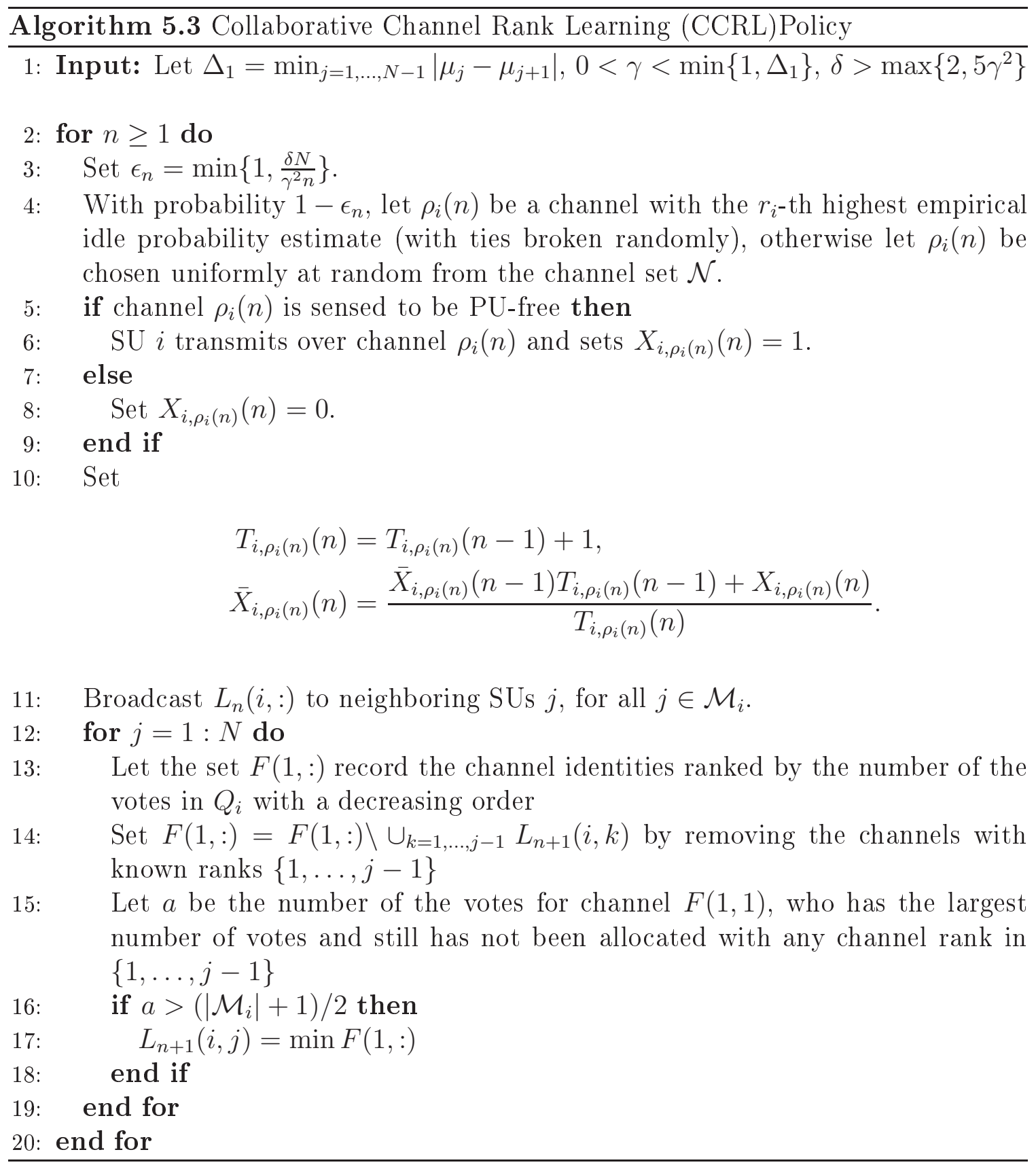

of each SU by only broadcasting its data summary $L_{n}(i,:)$ and it does not need to know the sensing observations or their empirical idle probability estimates of the channels. This advantage is especially important in today's environment, where users are increasingly wary about their own information and prefer not to directly share their real observations or statistical estimates. 
Table 5.3: Channel re-ranking example for a SU $i$

\begin{tabular}{|l|c|c|}
\hline Rank & $L_{n}(i,:)$ & $L_{n+1}(i,:)$ \\
\hline 1 & $L_{n}(i, 1)=1$ & $L_{n+1}(i, 1)=1$ \\
\hline 2 & $L_{n}(i, 2)=3$ & $L_{n+1}(i, 2)=2$ \\
\hline 3 & $L_{n}(i, 3)=4$ & $L_{n+1}(i, 3)=3$ \\
\hline 4 & $L_{n}(i, 4)=2$ & $L_{n+1}(i, 4)=4$ \\
\hline
\end{tabular}

In Section 5.3, we perform some simulations by applying our proposed collaborative learning policies and verify their performance.

\subsection{Simulation Results}

In this section, we compare and verify the performances of our proposed collaborative learning policies with the three-stage distributed channel access ranking and learning policy $\psi^{\mathrm{CARL}}$ in Chapter 4 by simulations. We first consider small size simple graphs, and then provide simulation results on large size random graphs.

\subsubsection{Small Size Graphs}

We apply our proposed three collaborative policies and the centralized policy $\psi^{\mathrm{CCA}}$ described in Section 4.2 on the three connected interference graphs, which are used in Section 4.4 (See Figure 4-3). We adopt the same parameter settings as in Section 4.4 of Chapter 4. In Algorithm 5.2, for each SU $i$, we set $\alpha_{i}=\frac{1}{\left|\mathcal{M}_{i}\right|+1}$ and $\alpha_{j}=\frac{1}{\left|\mathcal{M}_{i}\right|+1}$ for each $j \in \mathcal{M}_{i}$.

In Figures 5-2, 5-3 and 5-4, we observe that our proposed policies $\psi^{\mathrm{CAOCL}}, \psi^{\mathrm{CAMCL}}$ and $\psi^{\mathrm{CCRL}}$ outperform the policy $\psi^{\mathrm{CARL}}$ in all three interference graphs. The policies $\psi^{\mathrm{CAOCL}}$ and $\psi^{\mathrm{CAMCL}}$ even give a much smaller regret than the centralized policy 
$\psi^{\mathrm{CCA}}$. Moreover, we note that the policy $\psi^{\mathrm{CAOCL}}$ has the smallest regret than the other policies because it uses the sensing observations instead of channel estimates.

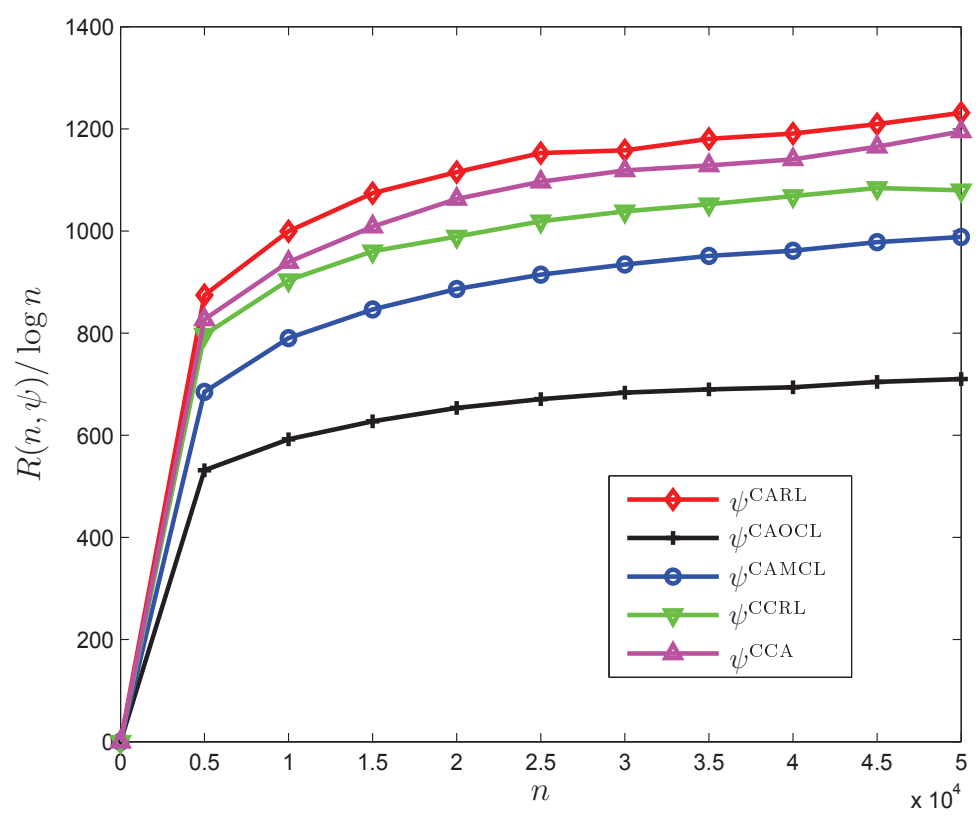

Figure 5-2: Normalized regret $\frac{R(n, \psi)}{\log n}$ vs. time slot $n$ with collaborative learning on ring graph.

\subsubsection{Large Size Random Graphs}

In this section, we consider large size random graphs that have $M=100 \mathrm{SUs}$ and $N=100$ orthogonal PU channels. The same idle probabilities of the PU channels and the same parameter settings are used as in Section 4.4 of Chapter 4 . We apply our proposed collaborative learning polices on two types of random graphs, which were also adopted in Chapter 4 . We evaluate the performance of the proposed policies on the Erdös-Rényi (ER) graphs and Random connection (RC) graphs.

In Figure 5-5 and Figure 5-6, we observe that the regrets using our proposed collaborative policies are again much smaller than the distributed policy $\psi^{\mathrm{CARL}}$ on 


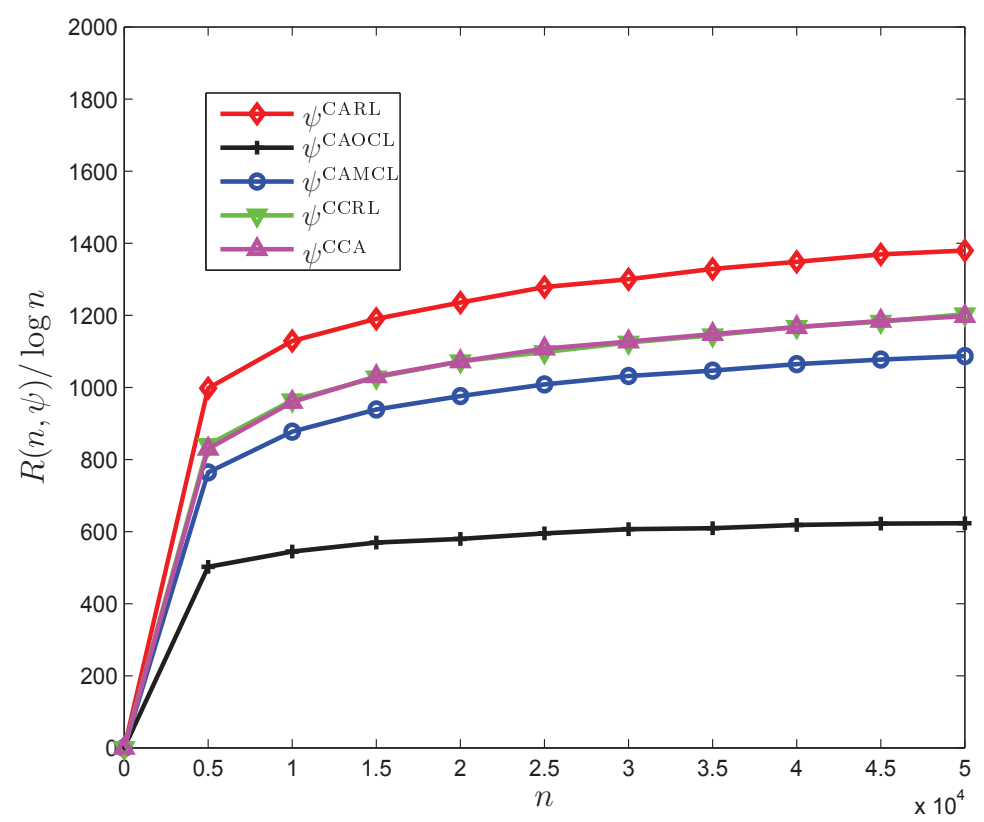

Figure 5-3: Normalized regret $\frac{R(n, \psi)}{\log n}$ vs. time slot $n$ with collaborative learning on grid graph.

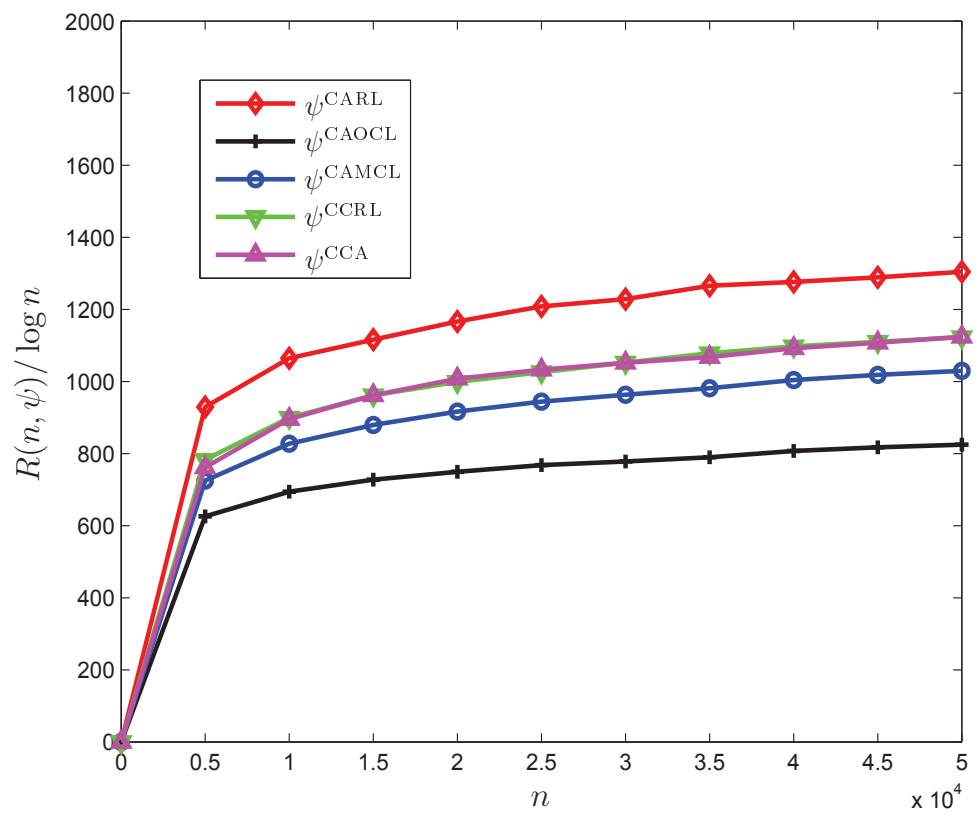

Figure 5-4: Normalized regret $\frac{R(n, \psi)}{\log n}$ vs. time slot $n$ with collaborative learning on randomly generated graph. 


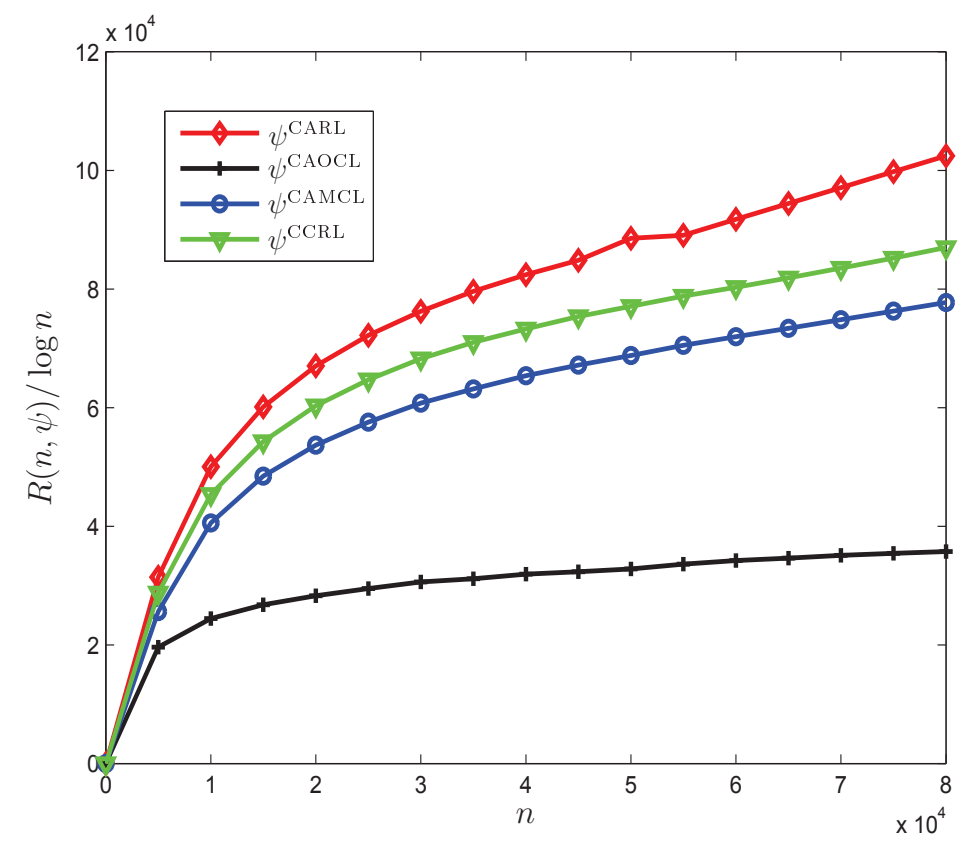

Figure 5-5: Normalized regret $\frac{R(n, \psi)}{\log n}$ vs. time slot $n$ with collaborative learning on ER graphs.

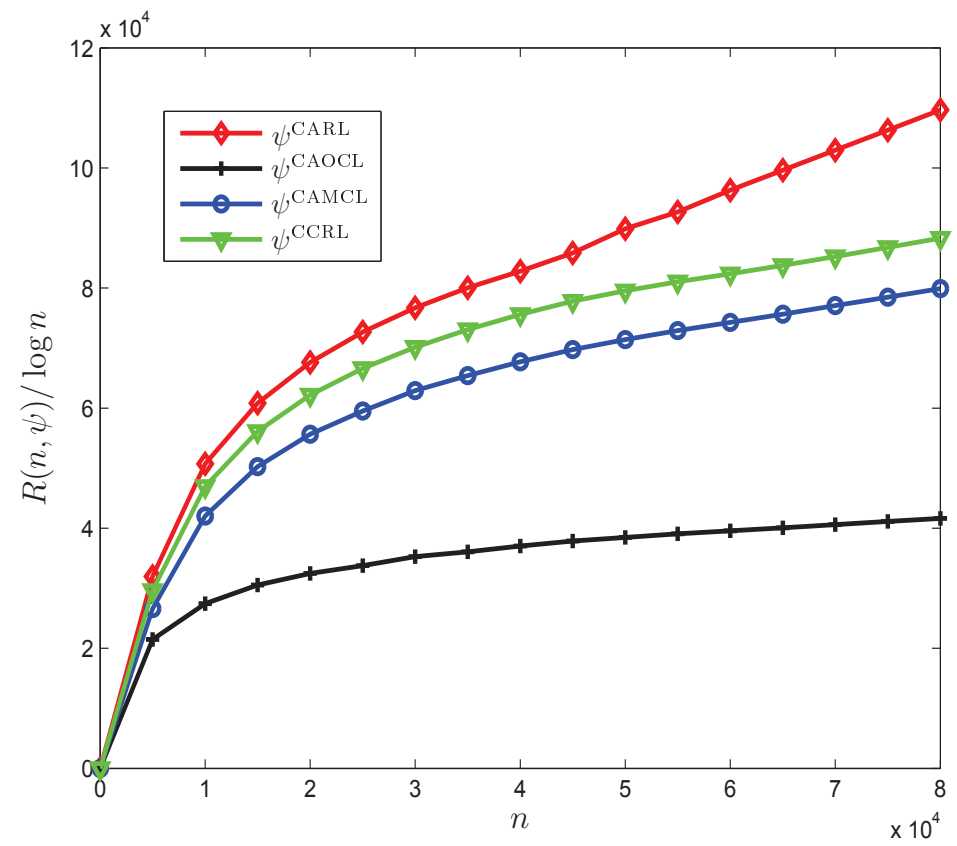

Figure 5-6: Normalized regret $\frac{R(n, \psi)}{\log n}$ vs. time slot $n$ with collaborative learning on RC graphs. 
the large size networks.

\subsection{Summary}

In this chapter, we have investigated the collaborative MAB problem for temporalspatial OSA in a large-scale network. To improve the learning speed, we have proposed three collaborative polices to learn the channel statistics by utilizing the sensing observations, empirical channel idle probabilities and rank orders of neighboring SUs. Simulation results suggest that our proposed collaborative policies outperform the distributed non-collaborative learning policy in terms of total regret of all SUs. 
THIS PAGE INTENTIONALLY LEFT BLANK 


\section{Chapter 6}

\section{Conclusion and Future Work}

\subsection{Conclusion}

In this thesis, we have investigated the problem of identifying temporal-spatial spectrum access opportunities. Several learning methods have been proposed to improve spectrum utilization in cognitive radio networks without knowing any information about the primary user's coverage region and channel idle probabilities.

In Chapter 3, by applying a distributed learning framework, we have developed a distributed boundary estimation algorithm to estimate the no-talk region of the PU for OSA in a cognitive radio network, and analyzed the trade-offs between the communication cost and estimation error of our proposed method. We derived approximate upper bounds for the communication cost and estimation error, and provided a method to compute the optimal SU density. Simulation results suggest that our proposed algorithm have lower estimation errors and better robustness compared to various other methods. 
In Chapter 4, we have investigated the temporal-spatial channel reuse problem within the no-talk region of a PU for OSA in cognitive radio networks using a multiuser MAB approach. We have proposed a centralized channel allocation policy for finding an optimal channel allocation and learning the statistics of the channels. We showed that this policy is order-optimal with logarithmic regret, but requires solving a NP-complete optimization problem at exponentially increasing time intervals. To avoid that and to overcome the requirement of centralized processing, we also proposed heuristic distributed policies, which however have linear regrets. The first distributed policy utilizes a distributed greedy graph coloring method and a distributed average consensus method in the learning process to find the optimal channel access rank for each SU. This requires synchronization amongst the SUs. In the second distributed policy, we let each SU determine their channel access ranks locally, which removes the requirement for synchronization. Simulation results suggest that our proposed policies outperform current policies in the literature, which do not consider spatial channel reuse.

In Chapter 5, we have investigated the collaborative MAB problem in a large-scale cognitive radio network. We proposed three collaborative polices to learn the channel statistics for temporal-spatial spectrum reuse by utilizing the sensing observations, empirical channel idle probability estimates and the estimated channel ranking of neighboring SUs. Simulations show that our proposed collaborative learning policies could largely improve the learning performance when comparing with a distributed policy without collaboration in the learning process. 


\subsection{Future Work}

In this work, we have assumed that the $\mathrm{PU}$ is static and the received interference of SUs at the PU do not vary over time. However, a more complex scenario where the users may be mobile is usually encountered in real life applications. Moreover, nowadays, large scale wireless sensor networks can be deployed for real-time monitoring applications, such as detecting of leakage of hazardous material, tracking forest fires and environmental phenomena. Consider a poisonous gas or plume monitoring application in a dynamical wireless network, estimating the changing boundary effectively and timely plays is of great importance for national security control. Therefore, a possible future work includes developing boundary estimation techniques for determining the no-talk region of dynamic PUs for the cases where PUs are mobile and where communication channels are time variant.

Moreover, within the no-talk region of the PU, we have assumed the channel availabilities are same for all SUs, which is quite idealized. Then, we plan to design policies for mobile SUs where channel availabilities differ across the SU network. Another interesting future research direction is to allow SUs to cooperatively learn channel access ranks and channel statistics by considering the trade-off between the learning performance and the required communication cost. Also, we may make natural extensions by studying some general cases where each $\mathrm{SU}$ is constrained to select a subset of channels and where the topology of SUs' interference network is dynamic. 
THIS PAGE INTENTIONALLY LEFT BLANK 


\section{Bibliography}

[1] Q. Zhao and B. Sadler. A survey of dynamic spectrum access. IEEE Signal Process. Mag., 24(3):79-89, 2007.

[2] I. F. Akyildiz, W.-Y. Lee, M. C. Vuran, and S. Mohanty. Next generation/dynamic spectrum access/cognitive radio wireless networks: A survey. Computer Networks Journal (Elsevier), 50:2127-2159, 2006.

[3] M. A. McHenry. NSF spectrum occupancy measurements projects summary. Shared Spectrum Company Report, 2005.

[4] S. Haykin. Cognitive radio: brain-empowered wireless communications. IEEE J. Sel. Areas Commun., 23(2):201-220, 2005.

[5] D. Makris, G. Gardikis, and A. Kourtis. Quantifying TV white space capacity: quantifying tv white space capacity:. IEEE Commun. Mag., 50(9):145-152, 2012.

[6] J. Unnikrishnan and V. V. Veeravalli. Cooperative sensing for primary detection in cognitive radio. IEEE J. Sel. Topics Signal Process., 2(1):18-27, 2008.

[7] R. Tandra, S. M. Mishra, and A. Sahai. What is a spectrum hole and what does it take to recognize one? Proc. IEEE, 97(5):824-848, 2009. 
[8] S. J. Shellhammer, S. Shankar, R. Tandra, and J. Tomcik. Performance of power detector sensors of DTV signals in IEEE 802.22 WRANs. In Proc. of Workshop on Technology and Policy for Accessing Spectrum, 2006.

[9] R. Tandra and A. Sahai. SNR walls for signal detection. IEEE J. Sel. Topics Signal Process., 2(1):4-17, 2008.

[10] Z. Li, F. R. Yu, and M. Huang. A cooperative spectrum sensing consensus scheme in cognitive radios. In Proc. of IEEE Joint Conf. of the IEEE Computer and Communications Societies, pages 2546-2550, 2009.

[11] W. Zhang and K. Letaief. Cooperative spectrum sensing with transmit and relay diversity in cognitive radio networks - [transaction letters]. IEEE Trans. Wireless Commun., 7(12):4761-4766, 2008.

[12] W. A. Gardner. Signal interception: a unifying theoretical framework for feature detection. IEEE Trans. Commun., 36(8):897-906, 1988.

[13] S. Geirhofer, L. Tong, and B. M. Sadler. Cognitive radios for dynamic spectrum access - dynamic spectrum access in the time domain: Modeling and exploiting white space. IEEE Commun. Mag., 45(5):66-72, 2007.

[14] C.-H. Huang and K.-C. Chen. Dual-observation time-division spectrum sensing for cognitive radios. IEEE Trans. Veh. Technol., 60(8):3712-3725, 2011.

[15] T. V. Krishna and A. Das. A survey on MAC protocols in OSA networks. Computer Networks, 53:1377-1394, 2009.

[16] J. Zhang, L. Qi, and H. Zhu. Optimization of MAC frame structure for opportunistic spectrum access. IEEE Trans. Wireless Commun., 11(6):2036-2045, 2012. 
[17] Y. Xu, J. Wang, Q. Wu, A. Anpalagan, and Y. D. Yao. Opportunistic spectrum access in unknown dynamic environment: a game-theoretic stochastic learning solution. IEEE Trans. Wireless Commun., 11(4):1380-1391, 2012.

[18] R. Tandra, A. Sahai, and V. Veeravalli. Unified space-time metrics to evaluate spectrum sensing. IEEE Commun. Mag., 49(3):54-61, 2011.

[19] F. Radlinski, M. Kurup, and T. Joachims. How does clickthrough data reflect retrieval quality? In Proc. of ACM Conf. on Information and Knowledge Management, pages 43-52, 2008.

[20] Y. Yue and T. Joachims. Interactively optimizing information retrieval systems as a dueling bandits problem. In Proc. of Int. Conf. on Machine Learning, pages 1201-1208, 2009.

[21] D. Agarwal, B. chung Chen, P. Elango, N. Motgi, S. taek Park, R. Ramakrishnan, S. Roy, and J. Zachariah. Online models for content optimization. In Proc. of the Neural Information Processing Systems Conf. (NIPS), pages 17-24, 2008.

[22] D. Chakrabarti, R. Kumar, F. Radlinski, and E. Upfal. Mortal multi-armed bandits. In Proc. of the Neural Information Processing Systems Conf. (NIPS), pages 273-280, 2008.

[23] T. Lu, D. Pál, and M. Pál. Contextual multi-armed bandits. In Proc. of Int. Conf. on Artificial Intelligence and Statistics (AISTATS), pages 485-492, 2010.

[24] V. Mnih, C. Szepesvári, and J.-Y. Audibert. Empirical bernstein stopping. In Proc. of Int. Conf. on Machine Learning, pages 672-679, 2008.

[25] J. B. Predd, S. R. Kulkarni, and H. V. Poor. A collaborative training algorithm for distributed learning. IEEE Trans. Inf. Theory, 55(4):1856-1871, 2009. 
[26] S. Tozlu, M. Senel, W. Mao, and A. Keshavarzian. Wi-Fi enabled sensors for Internet of Things: A practical approach. IEEE Commun. Mag., 50(6):134-143, 2012.

[27] C. Gavoille, R. Klasing, A. Kosowski, L. Kuszner, and A. Navarra. On the complexity of distributed graph coloring with local minimality constraints. Netw., $54(1): 12-19,2009$.

[28] F. Bénézit. Distributed Average Consensus for Wireless Sensor Networks. PhD thesis, École polytechnique fédérale de Lausanne, 2009.

[29] P. Auer, N. Cesa-Bianchi, and P. Fischer. Finite-time analysis of the multiarmed bandit problem. Mach. Learn., 47(2-3):235-256, 2002.

[30] A. Anandkumar, N. Michael, A. K. Tang, and A. Swami. Distributed algorithms for learning and cognitive medium access with logarithmic regret. IEEE J. Sel. Areas Commun., 29(4):731-745, 2011.

[31] K. Liu and Q. Zhao. Distributed learning in multi-armed bandit with multiple players. IEEE Trans. Signal Process., 58(11):5667-5681, 2010.

[32] E. Hillel, Z. Karnin, T. Koren, R. Lempel, and O. Somekh. Distributed exploration in multi-armed bandits. In Proc. of the Neural Information Processing Systems Conf. (NIPS), 2013.

[33] Y. Zhang, W. P. Tay, and K. H. Li. Distributed boundary estimation for spectrum sensing in cognitive radio networks. In Proc. of IEEE Wireless Communications and Networking Conf., 2013.

[34] Y. Zhang, W. P. Tay, K. H. Li, and D. Gaïti. Distributed boundary estimation for spectrum sensing in cognitive radio networks. IEEE J. Sel. Areas Commun., $32(11): 1-13,2014$. 
[35] Y. Zhang, W. P. Tay, K. H. Li, M. Esseghir, and D. Gaïti. Distributed opportunistic spectrum access with spatial reuse in cognitive radio networks. In Proc. of IEEE Global Conf. on Signal and Information Processing (GlobalSIP), 2014.

[36] Y. Zhang, W. P. Tay, K. H. Li, M. Esseghir, and D. Gaïti. Learning temporalspatial spectrum reuse. IEEE Trans. Commun., 64(7):3092-3103, 2016.

[37] Y. Zhang, W. P. Tay, K. H. Li, M. Esseghir, and D. Gaïti. Opportunistic spectrum access with temporal-spatial reuse in cognitive radio networks. In Accepted and Will Appear in Proc. of IEEE Conf. on Acoustics, Speech and Signal Processing (ICASSP), 2016.

[38] Spectrum Efficiency Working Group. FCC spectrum policy task force. Technical report, Federal Communications Commission (FCC), 2002.

[39] W. P. Tay, J. N. Tsitsiklis, and M. Z. Win. On the impact of node failures and unreliable communications in dense sensor networks. IEEE Trans. Signal Process., 56(6):2535 - 2546, June 2008.

[40] G. Kortuem, F. Kawsar, D. Fitton, and V. Sundramoorthy. Smart objects as building blocks for the Internet of Things. IEEE Internet Comput., 14(1):44-51, 2010.

[41] L. Xie, D.-H. Choi, S. Kar, and H. V. Poor. Fully distributed state estimation for wide-area monitoring systems. IEEE Trans. Smart Grid, 3(3):1154-1169, 2012.

[42] Y. Ding, Y. Jin, L. Ren, and K. Hao. An intelligent self-organization scheme for the Internet of Things. IEEE Computational Intelligence Magazine, 8(3):41-53, 2013. 
[43] W. P. Tay. Whose opinion to follow in multihypothesis social learning? A large deviations perspective. IEEE J. Sel. Topics Signal Process., 9(2):344 - 359, Mar. 2015.

[44] D. Duan, L. Yang, and J. C. Principe. Cooperative diversity of spectrum sensing for cognitive radio systems. IEEE Trans. Signal Process., 58(6):3218-3227, 2010.

[45] M. Z. Shakir, A. Rao, and M.-S. Alouini. Generalized mean detector for collaborative spectrum sensing. IEEE Trans. Commun., 61(4):1242-1253, 2013.

[46] G. Ozcan, M. cenk Gursoy, and S. Gezici. Error rate analysis of cognitive radio transmissions with imperfect channel sensing. IEEE Trans. Wireless Commun., 13(3):1642-1655, 2014.

[47] B. L. Mark and A. O. Nasif. Estimation of maximum interference-free power level for opportunistic spectrum access. IEEE Trans. Wireless Commun., $8(5): 2505-2513,2009$.

[48] S. M. Mishra. Maximizing available spectrum for cognitive radios. PhD thesis, UC Berkeley, 2010.

[49] S. M. Yu and S.-L. Kim. Optimal detection of spatial opportunity in wireless networks. IEEE Commun. Lett., 15(4):395-397, 2011.

[50] W. P. Tay. The value of feedback in decentralized detection. IEEE Trans. Inf. Theory, 58(12):7226 - 7239, Dec. 2012.

[51] M. Leng, W. P. Tay, T. Q. S. Quek, and H. Shin. Distributed local linear parameter estimation using Gaussian SPAWN. IEEE Trans. Signal Process., 63(1):244 - 257, Jan. 2015. 
[52] A. W. Min, X. Zhang, and K. G. Shin. Detection of small-scale primary users in cognitive radio networks. IEEE J. Sel. Areas Commun., 29:349-361, 2011.

[53] Q. Wu, G. Ding, J. Wang, and Y. Yao. Spatial-temporal opportunity detection for spectrum heterogeneous cognitive radio networks: Two-dimensional sensing. IEEE Trans. Wireless Commun., 12:516-526, 2013.

[54] Y. Ding, Y. Huang, G. Zeng, and L. Xiao. Using partially overlapping channels to improve throughput in wireless mesh networks. IEEE Trans. Mobile Comput., 11(11):1720-1733, 2012.

[55] R. Mochaourab, B. Holfeld, and T. Wirth. Distributed channel assignment in cognitive radio networks: Stable matching and walrasian equilibrium. IEEE Trans. Wireless Commun., 14(7):3924-3936, 2015.

[56] M. Félegyházi, M. Čagalj, and J.-P. Hubaux. Efficient MAC in cognitive radio systems: A game-theoretic approach. IEEE Trans. Wireless Commun., 8(4):1984-1995, 2009.

[57] M. Maskery, V. Krishnamurthy, and Q. Zhao. Decentralized dynamic spectrum access for cognitive radios: cooperative design of a non-cooperative game. IEEE Trans. Commun., 57(2):459-469, 2009.

[58] L. Cao and H. Zheng. Distributed spectrum allocation via local bargaining. In Proc. of IEEE Int. Conf. on Sensing, Communication, and Networking, 2005.

[59] M. Azarafrooz and R. Chandramouli. Distributed learning in secondary spectrum sharing graphical game. In Proc. of IEEE Global Telecomm. Conf., 2011.

[60] X. Chen and J. Huang. Distributed spectrum access with spatial reuse. IEEE J. Sel. Areas Commun., 31(3):593-603, 2013. 
[61] Y. Xu, Q. Wu, L. Shen, J. Wang, and A. Anpalagan. Opportunistic spectrum access with spatial reuse: graphical game and uncoupled learning solutions. IEEE Trans. Wireless Commun., 12(10):4814-4826, 2013.

[62] D. Kalathil, N. Nayyar, and R. Jain. Decentralized learning for multiplayer multiarmed bandits. IEEE Trans. Inf. Theory, 60(4):2331-2345, 2014.

[63] V. Kanade, Z. Liu, and B. Radunovic. Distributed non-stochastic experts. In Proc. of the Neural Information Processing Systems Conf. (NIPS), pages 260$268,2012$.

[64] I. Katzela and M. Naghshineh. Channel assignment schemes for cellular mobile telecommunication systems: a comprehensive survey. IEEE Personal Commun. Mag., 3(3):10-31, 1996.

[65] T. Yucek and H. Arslan. A survey of spectrum sensing algorithms for cognitive radio applications. IEEE Commun. Surveys Tuts., 11(1):116-130, 2009.

[66] E. Visotsky, S. Kuffner, and R. Peterson. On collaborative detection of TV transmissions in support of dynamic spectrum sharing. In Proc. of IEEE Int. Symposium New Frontiers in Dynamic Spectrum Access Networks (DySPAN), pages 338-345, 2005.

[67] A. Ghasemi and E. S. Sousa. Collaborative spectrum sensing for opportunistic access in fading environments. In Proc. of IEEE Int. Symposium New Frontiers in Dynamic Spectrum Access Networks, pages 131-136, 2005.

[68] B. L. To, M. Otmani, T. M. T. Nguyen, A. Fladenmuller, and G. Pujolle. Decentralized cooperative spectrum sensing for cognitive radio Ad-Hoc network: Hidden terminal awareness approach. In Proc. of Information Science, Signal Processing and their Applications, pages 146-151, 2012. 
[69] G. Ganesan and Y. Li. Cooperative spectrum sensing in cognitive radio: Part I: Two user networks. IEEE Trans. Wireless Commun., 6(6):2204-2213, 2007.

[70] G. Ganesan and Y. Li. Cooperative spectrum sensing in cognitive radio: Part II: Multiuser networks. IEEE Trans. Wireless Commun., 6(6):2214-2222, 2007.

[71] M. Vu, N. Devroye, and V. Tarokh. On the primary exclusive region of cognitive networks. IEEE Trans. Wireless Commun., 8(7):3380-3385, 2009.

[72] Z. Wei, Z. Feng, Q. Zhang, and W. Li. Three regions for space-time spectrum sensing and access in cognitive radio networks. In Proc. of IEEE Global Communications Conf., pages 1283-1288, 2012.

[73] Q. Li, Z. Feng, W. Li, and T. A. Gulliver. Joint temporal and spatial spectrum sharing in cognitive radio networks: A region-based approach with cooperative spectrum sensing. In Proc. of IEEE Wireless Communications and Networking Conf. (WCNC), pages 620-625, 2013.

[74] R. Nowak and U. Mitra. Boundary estimation in sensor networks: Theory and methods. In Proc. of Information Processing in Sensor Networks, pages 80-95, 2003.

[75] K. K. Chintalapudi and R. Govindan. Localized edge detection in sensor fields. In Proc. of IEEE Int. Workshop on Sensor Network Protocols and Applications, pages 59-70, 2003.

[76] Q. Fang, J. Gao, and L. J. Guibas. Locating and bypassing routing holes in sensor networks. In Proc. of IEEE Joint Conf. of the IEEE Computer and Communications Societies, pages 2458-2468, 2004. 
[77] S. P. Fekete, A. Kröller, D. Pfisterer, S. Fischer, and C. Buschmann. Neighborhood-based topology recognition in sensor networks. In Proc. of Int. Workshop on Algorithms for Sensor Systems, pages 123-136, 2004.

[78] S. P. Fekete, M. Kaufmann, A. Kröller, and K. A. Lehmann. A new approach for boundary recognition in geometric sensor networks. In Proc. of Canadian Conf. on Computational Geometry, pages 84-87, 2005.

[79] R. Ghrist and A. Muhammad. Coverage and hole-detection in sensor networks via homology. In Proc. of Int. Symposium Information Processing in Sensor Networks, pages 254-260, 2005.

[80] Y. Yang, Y. Liu, Q. Zhang, and L. Ni. Cooperative boundary detection for spectrum sensing using dedicated wireless sensor networks. In Proc. of IEEE Joint Conf. of the IEEE Computer and Communications Societies, pages 1-9, 2010.

[81] Y. Wang, J. Gao, and J. S. Mitchell. Boundary recognition in sensor networks by topological methods. In Proc. of Int. Conf. on Mobile Computing and Networking, pages 122-133, 2006.

[82] J. A. Suykens and J. Vandewalle. Least squares support vector machine classifiers. Neural Processing Letters, 9(3):293-300, 1999.

[83] B. Krishnamachari and S. Iyengar. Distributed Bayesian algorithms for faulttolerant event region detection in wireless sensor networks. IEEE Trans. Comput., 53(3):241-250, 2004.

[84] Q. Chen, K.-Y. Lam, and P. Fan. Comments on "Distributed Bayesian algorithms for fault-tolerant event region detection in wireless sensor networks". IEEE Trans. Comput., 54(9):1182-1183, 2005. 
[85] S. Duttagupta, K. Ramamritham, and P. Kulkarni. Tracking dynamic boundaries using sensor network. IEEE Trans. Parallel Distrib. Syst., 22(10):17661774, 2011.

[86] W. H. Press. Bandit solutions provide unified ethical models for randomized clinical trials and comparative effectiveness research. Proceedings of the National Academy of Sciences, 106(52):22387-22392, 2009.

[87] M. A. Bean. Probability: The Science of Uncertainty: with Applications to Investments, Insurance, and Engineering. Brooks Cole American Mathematical Society, 2009.

[88] B. Awerbuch and R. D. Kleinberg. Addaptive routing with end-to-end feedback: distributed learning and geometric approaches. In Proc. of ACM Symposiuim on Theory of Computing (STOC), pages 45-53, 2004.

[89] R. Kleinberg and T.Leighton. The value of knowing a demand curve: bounds on regret for online posted-price auctions. In Proc.of IEEE Symposium on Foundations of Computer Science, pages 594-605, 2003.

[90] P. Whittle. Restless bandits: Activity allocation in a changing world. Journal of Applied Probability, 25:287-298, 1988.

[91] V. Krishnamurthy and R. Evans. Hidden markov model multiarm bandits: a methodology for beam scheduling in multitarget tracking. IEEE Trans. Signal Process., 49(12):2893-2908, 2001.

[92] J. L. Ny, M. Dahleh, and E. Feron. Multi-agent task assignment in the bandit framework. In Proc. of IEEE Conf. on Decision and Control, 2006.

[93] T. L. Lai and H. Robbins. Asymptotically efficient adaptive allocation rules. Advances in Applied Mathematics, 6(1):4-22, 1985. 
[94] U. Syed, A. Slivkins, and N. Mishra. Adapting to the shifting intent of search queries. In Proc. of the Neural Information Processing Systems Conf. (NIPS), 2009.

[95] M. Kapralov and R. Panigrahy. Prediction strategies without loss. In Proc. of the Neural Information Processing Systems Conf. (NIPS), 2011.

[96] A. Agarwal, D. Hsu, S. Kale, J. Langford, L. Li, and R. E. Schapire. Taming the monster: A fast and simple algorithm for contextual bandits. In Proc. of Int. Conf. on Machine Learning, 2014.

[97] O. Besbes, Y. Gur, and A. Zeevi. Stochastic multi-armed-bandit problem with non-stationary rewards. In Proc. of the Neural Information Processing Systems Conf. (NIPS), 2014.

[98] M. Babaioff, S. Dughmi, R. Kleinberg, and A. Slivkins. Dynamic pricing with limited supply. In Proc. of ACM Conf. on Electronic Commerce, pages 74-91, 2012.

[99] A. Badanidiyuru, R. Kleinberg, and A. Slivkins. Bandits with knapsacks: Dynamic procurement for crowdsourcing. In Symposium on Foundations of Computer Science, 2013.

[100] Y. Gai and B. Krishnamachari. Distributed stochastic online learning policies for opportunistic spectrum access. IEEE Trans. Signal Process., 2014.

[101] G. Linden, B.Smith, and J. York. Amazon.com recommendations: item-to-item collaborative filtering. IEEE Internet Comput., 7(1):76-80, 2003.

[102] X. Su and T. M.Khoshgoftaar. A survey of collaborative filtering techniques. Advances in Artificial Intelligence, 2009, 2009. 
[103] S. Balakrishnan and S. Chopra. Collaborative ranking. In Proc. of ACM Int. Conf. on Web Search and Data Mining, pages 143-152, 2012.

[104] S. Brin and L. Page. The anatomy of a large-scale hypertextual web search engine. Computer Networks and ISDN Systems, 30(1-7):107-117, 1998.

[105] G. Zacharia, A. Moukas, and P. Maes. Collaborative reputation mechanisms for electronic marketplaces. Decision Support Systems - Special issue for business to business electronic commerce, issues and solutions, 29(4):371-388, 2000.

[106] H. Li. Customer reviews in spectrum: Recommendation system in cognitive radio networks. In Proc. of IEEE Symposium on New Frontiers in Dynamic Spectrum, pages 1-9, 2010.

[107] X. Chen, J. Huang, and H. Li. Adaptive channel recommendation for opportunistic spectrum access. IEEE Trans. Mobile Comput., 12(9):1788-1800, 2013.

[108] B. Awerbuch and R. Kleinberg. Competitive collaborative learning. Journal of Computer and System Sciences, 74(8):1271-1288, 2008.

[109] V. Gabillon, M. Ghavamzadeh, A. Lazaric, and S. Bubeck. Multi-bandit best arm identification. In Proc. of the Neural Information Processing Systems Conf. (NIPS), 2011.

[110] P. Auer, N. Cesa-Bianchi, Y. Freund, and R. E. Schapire. Gambling in a rigged casino: The adversarial multi-armed bandit problem. In Proc. of Symposium on Foundations of Computer Science, pages 322-331, 1995.

[111] O. Dekel, R. Gilad-Bachrach, O. Shamir, and L. Xiao. Optimal distributed online prediction using mini-batches. Journal of Machine Learning Research, 13(1):165-202, 2012. 
[112] A. Agarwal and J. C. Duchi. Distributed delayed stochastic optimization. In Proc. of IEEE Conf. on Decision and Control, pages 5451-5452, 2012.

[113] H. D. III, J. M. Phillips, A. Saha, and S. Venkatasubramanian. Efficient protocols for distributed classification and optimization. In Algorithmic Learning Theory, volume 7568, pages 154-168. Springer Berlin Heidelberg, 2012.

[114] D. Cabric, I. O'Donnell, M.-W. Chen, and R. Brodersen. Spectrum sharing radios. IEEE Circuits Syst. Mag., 6(2):30-45, 2006.

[115] R. Durrett. Probability: Theory and Examples. Belmont, CA: Duxbury, 3 edition, 2004.

[116] V. N. Vapnik. Statistical learning theory. Wiley, New York, 1998.

[117] R. Rom and M. Sidi. Multiple Access Protocols: Performance and Analysis. Springer London, 2011.

[118] I. Steinwart, D. Hush, and C. Scovel. An explicit description of the reproducing kernel Hilbert spaces of Gaussian RBF kernels. IEEE Trans. Inf. Theory, 52(10):4635-4643, 2006.

[119] Y. Censor and S. A. Zenios. Parallel Optimization: Theory, Algorithms, and Applications. New York: Oxford University Press, 1997.

[120] B. Schölkopf, R. Herbrich, and A. J. Smola. A generalized Representer Theorem. In Proc. of Conf. on Computational Learning Theory and 5th European Conf. on Computational Learning Theory, pages 416-426, 2001.

[121] J. J. Caffery. Wireless Location in CDMA Cellular Radio Systems. Boston : Kluwer Academic, 2000.

[122] C. Badea, S. Grivaux, and V. Müller. The rate of convergence in the method of alternating projections. St. Petersburg Math. J, 23:413-434, 2012. 
[123] L. Debnath and P. Mikusinski. Introduction to Hilbert spaces with applications. Academic Press, San Diego, CA, second edition, 1999.

[124] M. Z. Win, P. C. Pinto, and L. A. Shepp. A mathematical theory of network interference and its applications. Proc. IEEE, 97(2):205-230, 2009.

[125] C. han Lee and M. Haenggi. Interference and outage in poisson cognitive networks. IEEE Trans. Wireless Commun., 11(4):1392-1401, 2012.

[126] D. Stoyan, W. S. Kendall, and J. Mecke. Stochastic Geometry and its Applications. John Wiley \& Sons, Chichester, 1995.

[127] O. Bousquet and A. Elisseeff. Stability and generalization. Journal of Machine Learning Research, 2:499-526, 2002.

[128] Y.-C. Liang, Y. Zeng, E. C. Peh, and A. T. Hoang. Sensing-throughput tradeoff for cognitive radio networks. IEEE Trans. Wireless Commun., 7(4):1326-1337, 2008.

[129] W. C. Y. Lee. Lee's model [cellular radio path loss prediction]. In Proc. of IEEE Vehicular Technology Conf., pages 343-348, 1992.

[130] J. Fan, D. K. Y. Yau, A. K. Elmagarmid, and M. Walid G. Aref. Automatic image segmentation by integrating color-edge extraction and seeded region growing. IEEE Trans. Image Process., 10(10):1454-1466, 2001.

[131] R. M. Karp. Reducibility among combinatorial problems. Complexity of Computer Computations, pages 85-103, 1972.

[132] L. A. Wolsey. Integer Programming. Wiley-Interscience, 1998.

[133] N. Deo. Graph Theory with Applications to Engineering and Computer Science. PHI Learning Pvt. Ltd., 2004. 
[134] D. Brélaz. New methods to color the vertices of a graph. Commun. ACM, 22(4):251-256, 1979.

[135] E. Ruzgar and O. Dagdeviren. Performance evaluation of distributed synchronous greedy graph coloring algorithms on wireless ad hoc and sensor networks. Int. J. of Comput. Netw. and Commun., 5(2):169-179, 2013.

[136] A. Kosowski and K. Manuszewski. Classical coloring of graphs. American Mathematical Society, pages 1-19, 2004.

[137] A. Olshevsky. Linear time average consensus on fixed graphs and implications for decentralized optimization and multi-agent control. preprint, 2015.

[138] P. Erdös and A. Rényi. On random graphs. Publicationes Mathematicae Debrecen, 6:290-297, 1959. 


\section{Appendix A}

\section{List of Publications}

\section{Journals}

1. Y. Zhang, W. P. Tay, K. H. Li and D. Gaïti. Distributed Boundary Estimation for Spectrum Sensing in Cognitive Radio Networks. IEEE Journal on Selected Areas in Communications., 32(11):1961-1973, 2014.

2. Y. Zhang, W. P. Tay, K. H. Li, M. Esseghir and D. Gaïti. Learning TemporalSpatial Spectrum Reuse. IEEE Trans. on Communications., 64(7):3092-3103, 2016.

\section{Conferences}

1. Y. Zhang, W. P. Tay, K. H. Li and D. Gaïti. Distributed Boundary Estimation for Spectrum Sensing in Cognitive Radio Networks. In Proc. IEEE Wireless Communications and Networking Conference, 2013. 
2. Y. Zhang, W. P. Tay, K. H. Li, M. Esseghir and D. Gaïti. Distributed Opportunistic Spectrum Access with Spatial Reuse in Cognitive Radio Networks. In Proc. IEEE Global Conference on Signal and Information Processing, 2014.

3. Y. Zhang, W. P. Tay, K. H. Li, M. Esseghir and D. Gaïti. Opportunistic Spectrum Access with Temporal-Spatial Reuse in Cognitive Radio Networks. In Proc. IEEE Conference on Acoustics, Speech and Signal Processing, 2016. 